J. DIFFERENTIAL GEOMETRY

73 (2006) 263-317

\title{
COMPACTIFICATIONS OF LOCALLY SYMMETRIC SPACES
}

\author{
Armand Borel \& Lizhen Ji
}

\begin{abstract}
Let $G$ be the real locus of a connected semisimple linear algebraic group $\mathbf{G}$ defined over $\mathbb{Q}$, and $\Gamma \subset \mathbf{G}(\mathbb{Q})$ an arithmetic subgroup. Then the quotient $\Gamma \backslash G$ is a natural homogeneous space, whose quotient on the right by a maximal compact subgroup $K$ of $G$ gives a locally symmetric space $\Gamma \backslash G / K$. This paper constructs several new compactifications of $\Gamma \backslash G$. The first two are related to the Borel-Serre compactification and the reductive Borel-Serre compactification of the locally symmetric space $\Gamma \backslash G / K$; in fact, they give rise to alternative constructions of these known compactifications. More importantly, the compactifications of $\Gamma \backslash G$ imply extension to the compactifications of homogeneous bundles on $\Gamma \backslash G / K$, and quotients of these compactifications under non-maximal compact subgroups $H$ provide compactifications of period domains $\Gamma \backslash G / H$ in the theory of variation of Hodge structures. Another compactification of $\Gamma \backslash G$ is obtained via embedding into the space of closed subgroups of $G$ and is closely related to the constant term of automorhpic forms, in particular Eisenstein series.
\end{abstract}

\section{Introduction}

Let $\mathbf{G}$ be a semisimple linear connected algebraic group defined over $\mathbb{Q}, \Gamma \subset \mathbf{G}(\mathbb{Q})$ an arithmetic subgroup. Let $G=\mathbf{G}(\mathbb{R})$ be the real locus of $\mathbf{G}$, which is a Lie group with finitely many connected components. Let $K \subset G$ be a maximal compact subgroup of $G$. Then $X=G / K$ is a symmetric space of noncompact type. There are two natural spaces associated with the pair $(\Gamma, G)$ : a homogeneous space $\Gamma \backslash G$ and a locally symmetric space $\Gamma \backslash X=\Gamma \backslash G / K$. For many interesting natural subgroups $\Gamma$, for example, when $\mathbf{G}=\mathrm{SL}(n)$ and $\Gamma=\mathrm{SL}(n, \mathbb{Z})$, both $\Gamma \backslash G$ and $\Gamma \backslash G / K$ are non-compact.

Compactifications of $\Gamma \backslash X$ have been studied intensively from different points of view; for example, the Borel-Serre compactification $[\mathbf{B S}]$, the

The second author was artially supported by NSF grants and an Alfred P. Sloan Research Fellowship.

Received 03/21/2003. 
reductive Borel-Serre compactification $[\mathbf{Z u 1}],[\mathbf{G H M}]$, the Baily-Borel compactification $[\mathbf{B B}]$, and the geodesic compactification and others $[\mathbf{J M}]$, while much less attention has been paid to those of $\Gamma \backslash G$. On the other hand, $\Gamma \backslash G$ admits a right $G$-action and is the natural underlying space for automorphic representations, and automorphic forms of different $K$-types for $\Gamma$ can be studied simultaneously on $\Gamma \backslash G$ (see $[\mathbf{J i}]$ ). Furthermore, compactifications of $\Gamma \backslash G$ can be used to extend homogeneous bundles over $\Gamma \backslash X$ to compactifications of $\Gamma \backslash X$ (see Proposition $4.14)$, which was studied in $[\mathbf{Z u 3}]$. In this paper, we study systematically compactifications of $\Gamma \backslash G$ and obtain new constructions of the Borel-Serre and the reductive Borel-Serre compactifications of $\Gamma \backslash X$.

We give two methods to compactify $\Gamma \backslash G$. The first one is a modification of the approach in $[\mathbf{B S}]$, and we use it to construct two compactifications $\Gamma \backslash \bar{G}^{B S}$ and $\Gamma \backslash \bar{G}^{R B S}$, whose quotients by $K$ on the right give the compactifications $\Gamma \backslash \bar{X}^{B S}$ and $\Gamma \backslash \bar{X}^{R B S}$. In fact, this modified method also gives a slightly different construction of the Borel-Serre compactification $\Gamma \backslash \bar{X}^{B S}$ which avoids the introduction of spaces of S-type in [BS], and a direct construction of $\Gamma \backslash \bar{X}^{R B S}$ without using $\Gamma \backslash \bar{X}^{B S}$ as was defined in $[\mathbf{Z u 1}]$. The basic differences between this method and the original method in [BS] are: (1) to replace the geodesic action on $X$ by an action defined directly in terms of Langlands decomposition, since there is no canonically defined geodesic action on $G,(2)$ to start with the boundary components instead of corners and hence avoid the spaces of S-type which are needed in the inductive proofs in [BS]. See $\S 3$ for more details about relations between them and other applications of this modified method. Similar compactifications of $\Gamma \backslash G$ have been obtained independently in $[\mathbf{K U}]$.

The second method is to embed $\Gamma \backslash G$ equivariantly into some compact $G$-spaces such that the closure gives a compactification. We construct such a compactification $\Gamma \backslash \bar{G}^{s b}$ when $\Gamma$ is a maximal arithmetic subgroup using the space $\mathcal{S}(G)$ of closed subgroups of $G$, which is a compact $G$ space.

A natural approach to understand compactifications of $\Gamma \backslash G$ is to relate them to compactifications of $\Gamma \backslash X$. When $\Gamma$ is torsion free, the space $\Gamma \backslash G$ is a $K$-principal bundle over $\Gamma \backslash X=\Gamma \backslash G / K$, and it is conceivable that a compactification of $\Gamma \backslash X$ can be lifted to a compactification of $\Gamma \backslash G$ which admits a right $K$-action. A natural question is whether the $K$-principal bundle structure can be extended to the compactification. The answer is affirmative for $\Gamma \backslash \bar{G}^{B S}$ and $\Gamma \backslash \bar{G}^{R B S}$. For the purpose of the representation theory and automorphic forms, it is also natural to ask whether the right $G$-action on $\Gamma \backslash G$ extends continuously to its compactifications. It turns out that the $G$-action extends to $\Gamma \backslash \bar{G}^{R B S}$ 
but not to $\Gamma \backslash \bar{G}^{B S}$. A natural explanation is given by the fact that the $G$-compactification $\Gamma \backslash \bar{G}^{s b}$ is closely related to $\Gamma \backslash \bar{G}^{R B S}$.

Compactifications $\Gamma \backslash \bar{G}^{B S}$ and $\Gamma \backslash \bar{G}^{R B S}$ also give compactifications of period domains, which were sought after in $[\mathbf{G r}]$. Period domains in the theory of variation of Hodge structure are of the form $\Gamma \backslash G / H$ for some (compact) subgroups $H$ of $K$. Since the right $K$-action on $\Gamma \backslash G$ extends to $\Gamma \backslash \bar{G}^{B S}$ and $\Gamma \backslash \bar{G}^{R B S}$, we obtain compactifications of $\Gamma \backslash G / H$ by taking the quotients $\left(\Gamma \backslash \bar{G}^{B S}\right) / H,\left(\Gamma \backslash \bar{G}^{R B S}\right) / H$.

Motivated by the Oshima compactification $\bar{X}^{O}$ [Os1] and its applications to harmonic analysis $[\mathbf{O s} 3]$, we show that $\Gamma \backslash \bar{G}^{B S}$ is a real analytic manifold with corners and $2^{r}$-copies of $\Gamma \backslash \bar{G}^{B S}$ can be self-glued into a closed analytic manifold $\Gamma \backslash \bar{G}^{B S}$ which admits a natural $(\mathbb{Z} / 2 \mathbb{Z})^{r}$-action and whose quotient by $(\mathbb{Z} / 2 \mathbb{Z})^{r}$ is $\Gamma \backslash \bar{G}^{B S}$, where $r$ is the $\mathbb{Q}$-rank of G. Similarly, using the result in $[\mathbf{B S}]$ that $\Gamma \backslash \bar{X}^{B S}$ is a real analytic manifold with corners, we can show that $2^{r}$-copies of $\Gamma \backslash \bar{X}^{B S}$ can be self-glued into a closed analytic manifold $\Gamma \backslash \bar{X}^{B S O}$. The space $\Gamma \backslash \bar{X}^{B S O}$ has been constructed independently by Weselmann in the adelic case in $[\mathbf{W e}]$ to study the trace of Hecke operators. The basic idea is that functions on $\Gamma \backslash \bar{X}^{B S}$ can be lifted $(\mathbb{Z} / 2 \mathbb{Z})^{r}$-equivariant functions on the closed manifold $\Gamma \backslash \bar{X}^{B S O}$.

This paper is organized as follows. In $\S 2$, we recall several basic facts about parabolic $\mathbb{Q}$-subgroups; in particular, the Langlands decomposition, liftings of the Levi quotient, and the reduction theory. In $\S 3$ and $\S 4$, we use a modified version of the method in $[\mathbf{B S}]$ to define compactifications $\Gamma \backslash \bar{G}^{B S}$ and $\Gamma \backslash \bar{G}^{R B S}$ of $\Gamma \backslash G$. When $\Gamma$ is neat, $\Gamma \backslash \bar{G}^{B S}$ is a principal $K$-bundle over the Borel-Serre compactification $\Gamma \backslash \bar{X}^{B S}$, and $\Gamma \backslash \bar{G}^{R B S}$ is a principal $K$-bundle over $\Gamma \backslash \bar{X}^{R B S}$. This is the reason for the above notations $\Gamma \backslash \bar{G}^{B S}$ and $\Gamma \backslash \bar{G}^{R B S}$.

Since $\Gamma \backslash \bar{G}^{B S}$ and $\Gamma \backslash \bar{G}^{R B S}$ are obtained by adding ideal points at infinity, a natural question is whether we can embed $\Gamma \backslash G$ into a compact topological space such that the closure of $\Gamma \backslash G$ gives the same compactification. In $\S 5$, we carry out such a construction when $\Gamma$ is a maximal discrete subgroup of $G$. In this case, we can embed $\Gamma \backslash G$ into the space $\mathcal{S}(G)$ of closed subgroups in $G$. The closure of $\Gamma \backslash G$ in $\mathcal{S}(G)$ defines a compactification of $\Gamma \backslash G$, called the subgroup compactification and denoted by $\Gamma \backslash \bar{G}^{s b}$. We study the boundary subgroups and show that $\Gamma \backslash \bar{G}^{s b}$ is isomorphic to $\Gamma \backslash \bar{G}^{R B S}$ under some assumptions. In general, $\Gamma \backslash \bar{G}^{s b}$ is similar to the maximal Satake compactification of $\Gamma \backslash X$ in the 
sense that only the semisimple part of the Levi quotient of parabolic $\mathbb{Q}$-subgroups appears in the boundary.

In $\S 6$, we show $\Gamma \backslash \bar{G}^{B S}$ is a real analytic manifold with corners. This is similar to the result in $[\mathbf{B S}]$ that $\Gamma \backslash \bar{X}^{B S}$ is a real analytic manifold with corners. In $\S 7$, we describe a general method, due to Melrose [Me], to self-glue a manifold with corners into a closed manifold. It is applied in $\S 8$ to $\Gamma \backslash \bar{X}^{B S}, \Gamma \backslash \bar{G}^{B S}$ and yields $\Gamma \backslash \bar{G}^{B S O}$ and $\Gamma \backslash \bar{X}^{B S O}$, which are analogues of the Oshima compactification $\bar{X}^{O}$ [Os1].

Finally, we comment briefly on some motivations of the results in this paper. In [Bo6], the first author studied simultaneously several compactifications of symmetric spaces. In particular, he initiated the study of the real locus of the wonderful compactification of symmetric varieties of De Concini and Procesi $[\mathbf{D P}]$ and related it to other compactifications such as the Satake compactifications [Sa1] and the Oshima compactification $\left[\right.$ Os1]; he also raised the question as to whether $\Gamma \backslash \bar{X}^{B S O}$ exists. The Oshima compactification of $X$ shows that the maximal Satake compactification is a real analytic manifold with corners; this analytic structure and the embedding into the Oshima compactification play an important role in proving the Helgason conjecture in $[\mathbf{K K}]$ and the program of Oshima to study representation theory via boundary values of differential equations as explained in [Os3]. The reductive Borel-Serre compactification $\Gamma \backslash \bar{X}^{R B S}$ is used in both [Zu1] and [GHM] and was defined as a quotient of $\Gamma \backslash \bar{X}^{B S}$ in $[\mathbf{Z u 1}]$. A natural question is to give a direct construction independent of $\Gamma \backslash \bar{X}^{B S}$. In [Ma], MacPherson studied $\Gamma \backslash X$ and its compactifications using the geometry of lattices in $\mathbb{R}^{n}$. Since $\Gamma \backslash G$ can be canonically mapped into the space of lattice subgroups of $G$, a natural problem is to study compactifications of $\Gamma \backslash G$ using this map, and $\S 5$ is a direct result of such a study.

Some of the results of this paper have been announced in [BJ1]. This paper, except $\S 7$ and the first part of $\S 5$, was mainly written up by the second author, who will bear the primary responsibility for it.

\section{Conventions.}

In this paper, a linear algebraic group defined over $\mathbb{Q}$ or $\mathbb{R}$ is denoted by a bold face letter and its group of real points by the corresponding Roman capital. For any $x, y \in G$, define

$$
x^{y}=y^{-1} x y, \quad{ }^{y} x=y x y^{-1} .
$$

The same notation applies when $x$ is replaced by a subset of $G$. Parabolic subgroups of $\mathbf{G}$ always refer to proper parabolic subgroups unless indicated otherwise.

Acknowledgment. We would like to thank an anonymous refreee for his extremely careful reading and many kind suggestions. 


\section{Parabolic subgroups and Langlands decomposition}

In this section, we recall some basic facts about parabolic $\mathbb{Q}$-subgroups of $\mathbf{G}$ and some discrete subgroups induced from an arithmetic subgroup $\Gamma$ of $\mathbf{G}(\mathbb{Q})$.

Let $r=r k_{\mathbb{Q}}(\mathbf{G})$ denote the $\mathbb{Q}$-rank of $\mathbf{G}$. Assume that $r>0$, i.e., $\Gamma \backslash G$ is noncompact. For any parabolic $\mathbb{Q}$-subgroup $\mathbf{P}$ of $\mathbf{G}$, let $\mathbf{N}_{P}$ be the unipotent radical of $\mathbf{P}$, and $\mathbf{L}_{P}=\mathbf{N}_{P} \backslash \mathbf{P}$ be the Levi quotient of $\mathbf{P}$. Then both $\mathbf{N}_{P}$ and $\mathbf{L}_{P}$ are rational algebraic groups. Let $N_{P}=\mathbf{N}_{P}(\mathbb{R})$, $P=\mathbf{P}(\mathbb{R}), L_{P}=\mathbf{L}_{P}(\mathbb{R})$ be their real loci. Let $\mathbf{S}_{P}$ be the split center of $\mathbf{L}_{P}$ over $\mathbb{Q}$, and $A_{P}$ the connected component of the identity in $\mathbf{S}_{P}(\mathbb{R})$. Let $\mathbf{M}_{P}=\cap_{\chi \in X\left(\mathbf{L}_{P}\right)}$ Ker $\chi^{2}$. Then $\mathbf{M}_{P}$ is an algebraic group defined over $\mathbb{Q}$. Let $M_{P}=\mathbf{M}_{P}(\mathbb{R})$. Then $L_{P}$ admits a decomposition

$$
L_{P}=A_{P} M_{P} \cong A_{P} \times M_{P} .
$$

For applications in later sections, we need to lift $L_{P}$ and its subgroups into $P$. Let $X$ be the symmetric space of maximal compact subgroups of $G=\mathbf{G}(\mathbb{R})$. Let $K$ be a maximal compact subgroup of $G$. Then $X \cong G / K$. Let $x_{0} \in X$ be the basepoint corresponding to $K$. The Cartan involution $\theta$ of $G$ associated with $K$ extends to an involution of $\mathbf{G}$. It is shown in [BS, 1.9] (see also [GHM, pp. 149-151]) that there exists a unique Levi subgroup $\mathbf{L}_{P, x_{0}}$ stable under the extended Cartan involution. The canonical projection $\pi_{P}: \mathbf{L}_{P, x_{0}} \rightarrow \mathbf{P} / \mathbf{N}$ yields an isomorphism of $\mathbf{L}_{P, x_{0}}$ onto $\mathbf{L}_{P}$. We let $i_{x_{0}}$ be the inverse to the restriction of $\pi_{P}$ to $L_{P, x_{0}}$. In particular, it is an isomorphism of $L_{P}$ onto $L_{P, x_{0}}$. We let $A_{P, x_{0}}$ and $M_{P, x_{0}}$ denote the images of $A_{P}$ and $M_{P}$ under $i_{x_{0}}$.

Note that though $\mathbf{L}_{P}, \mathbf{M}_{P}, \mathbf{S}_{P}$ are algebraic groups defined over $\mathbb{Q}$, their lifts $\mathbf{L}_{P, x_{0}}, \mathbf{M}_{P, x_{0}}, \mathbf{S}_{P, x_{0}}$ are not necessarily defined over $\mathbb{Q}$. Of course, they are defined over $\mathbb{R}$.

The lift $i_{x_{0}}\left(L_{P}\right)$ splits the exact sequence, $0 \rightarrow N_{P} \rightarrow P \rightarrow L_{P} \rightarrow 0$, and gives rise to the Langlands decomposition of $P$ :

$$
P=N_{P} A_{P, x_{0}} M_{P, x_{0}} \cong N_{P} \times A_{P, x_{0}} \times M_{P, x_{0}},
$$

i.e., for any $g \in P, g=n(g) a(g) m(g)$, where $n(g) \in N_{P}, a(g) \in$ $A_{P, x_{0}}, m(g) \in M_{P, x_{0}}$ are uniquely determined by $g$, and the map $g \rightarrow(n(g), a(g), m(g))$ gives a real analytic diffeomorphism between $P$ and $N_{P} \times A_{P, x_{0}} \times M_{P, x_{0}}$. The map $P \rightarrow N_{P} \times A_{P, x_{0}} \times M_{P, x_{0}}$ is equivariant with respect to the $P$-action defined on the right-hand side by

$$
n_{0} a_{0} m_{0}(n, a, m)=\left(n_{0}{ }^{a_{0} m_{0}} n, a_{0} a, m_{0} m\right)
$$

for $p=n_{0} a_{0} m_{0} \in P$. Since $G=P K$, the subgroup $P$ acts transitively on $X=G / K$, and the Langlands decomposition of $P$ gives the following horospherical decomposition of $X$ :

$$
X=N_{P} \times A_{P, x_{0}} \times X_{P, x_{0}},
$$


where $X_{P, x_{0}}=M_{P, x_{0}} / K \cap M_{P, x_{0}}$ is called the boundary symmetric space associated with $P$. The Langlands decomposition of $P$ also induces the following horospherical decomposition of $G$ :

$$
G=N_{P} A_{P, x_{0}} M_{P, x_{0}} K=N_{P} \times A_{P, x_{0}} \times M_{P, x_{0}} K,
$$

i.e., any element $g \in G$ can be written uniquely in the form $g=$ $n(g) a(g) m(g)$, where $n(g) \in N_{P}, a(g) \in A_{P, x_{0}}, m(g) \in M_{P, x_{0}} K$, and the map $g \mapsto(n(g), a(g), m(g))$ gives a real analytic diffeomorphism between $G$ and $N_{P} \times A_{P, x_{0}} \times M_{P, x_{0}} K$. In the following, $(n, a, m)$ is often identified with nam for simplicity.

The group $K \cap P$ is equal to $K \cap M_{P, x_{0}}$ and is a maximal compact subgroup of $P$ and of $M_{P, x_{0}}$. Its image in $M_{P}$ under the canonical projection will sometimes be denoted $M_{P} \cap K$. It is a maximal compact subgroup of $M_{P}$ and $X_{P}=M_{P} / K \cap M_{P}$ is the symmetric space of $M_{P}$. The projection $P \rightarrow N_{P} \backslash P$ identifies $M_{P, x_{0}}$ with $M_{P}$, and hence $X_{P, x_{0}}$ with $X_{P}$.

Decompositions similar to the above hold for reductive groups with finitely many connected components. This is important for the purpose of induction. In fact, though $\mathbf{G}$ is connected and semisimple, $\mathbf{M}_{P}$ is in general reductive with finitely many connected components. (See Remark 2.10 below.)

Lemma 2.1. For any other basepoint $x_{1}=p x_{0}=p K p^{-1} \in X$, where $p \in P$, the Levi subgroup $\mathbf{L}_{P, x_{1}}$ associated with the basepoint $x_{1}$ is $p \mathbf{L}_{P, x_{0}} p^{-1}$, and $A_{P, x_{1}}=p A_{P, x_{0}} p^{-1}, M_{P, x_{1}}=p M_{P, x_{0}} p^{-1}$. If $n$ is the $N_{P^{-}}$ component of $p$ in the Langlands decomposition of $P=N_{P} A_{P, x_{0}} M_{P, x_{0}}$, then $A_{P, x_{1}}=n A_{P, x_{0}} n^{-1}, M_{P, x_{1}}=n M_{P, x_{0}} n^{-1}$.

Proof. Let $\theta$ be the Cartan involution for the basepoint $x_{0}$. Then the Cartan involution for $x_{1}$ is given by $\operatorname{Int} p \circ \theta \circ \operatorname{Int} p^{-1}$. Since $p \in P$, $p L_{P, x_{0}} p^{-1}$ belongs to $P$ and is invariant under $\operatorname{Int} p \circ \theta \circ \operatorname{Int} p^{-1}$. This implies $p \mathbf{L}_{P, x_{0}} p^{-1}$ is the lift associated with $x_{1}$. The rest is clear. q.e.d.

Proposition 2.2. For any parabolic $\mathbb{Q}$-subgroup $\mathbf{P}$, there exists a basepoint $x_{1} \in X$ and a lift map $i_{x_{1}}$ such that $i_{x_{1}}$ is rational in the sense that the images $i_{x_{1}}\left(\mathbf{L}_{P}\right), i_{x_{1}}\left(\mathbf{M}_{P}\right), i_{x_{1}}\left(\mathbf{S}_{P}\right)$ are algebraic subgroups defined over $\mathbb{Q}$, and the lift $i_{x_{1}}$ is defined over $\mathbb{Q}$.

Proof. Since $\mathbf{P}$ is defined over $\mathbb{Q}$, there is a Levi subgroup $\mathbf{L}_{P}^{\prime}$ defined over $\mathbb{Q}$. Since all the Levi subgroups of $\mathbf{P}$ are conjugate under $N_{P}$, there exists $n \in N_{P}$ such that $\mathbf{L}_{P}^{\prime}=n i_{x_{0}}\left(\mathbf{L}_{P}\right) n^{-1}$. Let $x_{1}=n x_{0}$. Then the proof of the above lemma shows that $i_{x_{1}}\left(\mathbf{L}_{P}\right)=\mathbf{L}_{P}^{\prime}$. $\quad$ q.e.d.

Next we study dependence of the various decompositions on the choice of the basepoint $x_{0}$ and the lift map $i_{x_{0}}$. Let $x_{0}=K \in X$ be the basepoint above. The basepoint in $X_{P}$ corresponding to the 
subgroup $K \cap M_{P}$ is also denoted by $x_{0}$ for simplicity. For any other basepoint $x \in X$, write

$$
x=p_{0} x_{0}=n_{0} a_{0} m_{0} x_{0}
$$

where $p_{0} \in P, n_{0} \in N_{P}, a_{0} \in A_{P, x_{0}}, m_{0} \in M_{P, x_{0}}$, and $n_{0}, a_{0}$ are uniquely determined by $x$. By Lemma 2.1, the lifted subgroups associated with the basepoint $x$ are

$$
A_{P, x}=p_{0} A_{P, x_{0}} p_{0}^{-1}=n_{0} A_{P, x_{0}} n_{0}^{-1}, \quad M_{P, x}=n_{0} M_{P, x_{0}} n_{0}^{-1},
$$

since $A_{P, x_{0}}$ and $M_{P, x_{0}}$ commute. The maximal compact subgroup stabilizing the basepoint $x$ is $p_{0} K p_{0}^{-1}$. The following lemma describes how coordinates change in the horospherical decomposition of $X$ and $G$ with respect to different basepoints $x_{0}$ and $x=p_{0} x_{0}$.

\section{Lemma 2.3.}

1) The coordinates of $\left(n, a, m x_{0}\right) \in N_{P} \times A_{P, x_{0}} \times X_{P, x_{0}}=X$ in the horospherical decomposition of $X$ with respect to the basepoint $x=p_{0} x_{0}$ are given by

$$
\left(n n_{0}^{-1},{ }^{n_{0}}\left(a a_{0}^{-1}\right),{ }^{n_{0}}\left(m m_{0}^{-1}\right) x\right) \in N_{P} \times A_{P, x} \times X_{P, x},
$$

where $p_{0}=n_{0} a_{0} m_{0}, n_{0} \in N_{P}, a_{0} \in A_{P, x_{0}}, m_{0} \in M_{P, x_{0}}$.

2) The coordinates of $(n, a, m) \in N_{P} \times A_{P, x_{0}} \times M_{P, x_{0}} K$ in the horospherical decomposition of $G$ with respect to the basepoint $x=p_{0} x_{0}$ are given by

$$
\begin{gathered}
\left(n\left({ }^{a} n_{1}\right) n_{0}^{-1},{ }^{n_{0}}\left(a a_{1} a_{0}^{-1}\right),{ }^{p_{0}}\left(m_{0}^{-1} m_{1}\right)\right) \in N_{P} \times A_{P, x} \times{ }^{p_{0}}\left(M_{P, x_{0}} K\right), \\
\text { where } m p_{0}=\left(n_{1}, a_{1}, m_{1}\right) \in N_{P} \times A_{P, x_{0}} \times M_{P, x_{0}} K .
\end{gathered}
$$

Proof. For $\left(n, a, m x_{0}\right) \in N_{P} \times A_{P, x_{0}} \times X_{P, x_{0}}$, the corresponding point in $X$ is $n a m x_{0}$. Since

$$
n a m x_{0}=n a m m_{0}^{-1} a_{0}^{-1} n_{0}^{-1}\left(p_{0} x_{0}\right)=n n_{0}^{-1} \cdot{ }^{n_{0}}\left(a a_{0}^{-1}\right) \cdot{ }^{n_{0}}\left(m m_{0}^{-1}\right) \cdot x,
$$

it follows from Equation (2.4) that ${ }^{n_{0}}\left(a a_{0}^{-1}\right) \in A_{P, x},{ }^{n_{0}}\left(m m_{0}^{-1}\right) \in M_{P, x}$ whence part (1).

To prove part (2), we first compute the horospherical coordinates of $p_{0}^{-1}$ namp $_{0}$ with respect to the basepoint $x_{0}$. Then the conjugation by $p_{0}$ gives the horospherical coordinates of $g$ with respect to the basepoint $x=p_{0} x_{0}$. Since $p_{0}=n_{0} a_{0} m_{0}$,

$$
p_{0}^{-1} \operatorname{namp}_{0}=m_{0}^{-1} a_{0}^{-1} n_{0}^{-1} \operatorname{namp}_{0} .
$$


Write $m p_{0}=n_{1} a_{1} m_{1}$, where $n_{1} \in N_{P}, a_{1} \in A_{P, x_{0}}, m_{1} \in M_{P, x_{0}} K$. Then

$$
\begin{aligned}
p_{0}^{-1} \text { namp }_{0}= & m_{0}^{-1} a_{0}^{-1} n_{0}^{-1} \text { nan }_{1} a_{1} m_{1} \\
= & m_{0}^{-1} a_{0}^{-1} \cdot n_{0}^{-1} n\left(a n_{1} a^{-1}\right) \cdot a a_{1} m_{1} \\
= & \left(a_{0} m_{0}\right)^{-1}\left(n_{0}^{-1} n\left({ }^{a} n_{1}\right)\right)\left(a_{0} m_{0}\right) \cdot a a_{1} a_{0}^{-1} \cdot m_{0}^{-1} m_{1} \\
= & \left(\left(a_{0} m_{0}\right)^{-1}\left(n_{0}^{-1} n\left({ }^{a} n_{1}\right)\right)\left(a_{0} m_{0}\right), a a_{1} a_{0}^{-1}, m_{0}^{-1} m_{1}\right) \\
& \quad \in N_{P} \times A_{P, x_{0}} \times M_{P, x_{0}} K,
\end{aligned}
$$

where we have used that fact that $p_{0} \in P$ and hence $m_{0} \in M_{P, x_{0}}$. This implies that the horospherical coordinates of $n a m=p_{0}\left(p_{0}^{-1} n a m p_{0}\right) p_{0}^{-1}$ with respect to the basepoint $x=p_{0} x_{0}$ are

$$
\begin{aligned}
& \left(p_{0}\left(a_{0} m_{0}\right)^{-1}\left(n_{0}^{-1} n\left({ }^{a} n_{1}\right)\left(a_{0} m_{0}\right) p_{0}^{-1}, p_{0} a a_{1} a_{0} p_{0}^{-1}, p_{0} m_{0}^{-1} m_{1} p_{0}^{-1}\right)\right. \\
& =\left(n\left({ }^{a} n_{1}\right) n_{0}^{-1}, n_{0}\left(a a_{1} a_{0}\right) n_{0}^{-1}, p_{0} m_{0}^{-1} m_{1} p_{0}^{-1}\right) \\
& =\left(n\left({ }^{a} n_{1}\right) n_{0}^{-1},{ }^{n_{0}}\left(a a_{1} a_{0}\right),{ }^{p_{0}}\left(m_{0}^{-1} m_{1}\right)\right)
\end{aligned}
$$

and completes the proof of part (2).

q.e.d.

2.4. In the following, the reference to the basepoint $x_{0}$ in various subscripts will be omitted unless needed. The unipotent subgroup $N_{P}$ is a normal subgroup in $P$ and $A_{P}$ acts on it by conjugation and on its Lie algebra $\mathfrak{n}_{P}$ by the adjoint representation. We let $\Phi\left(A_{P}, P\right)$ be the set of characters of $A_{P}$ in $\mathfrak{n}_{P}$, the "roots of $P$ with respect to $A_{P}$ ". The value of $\alpha \in \Phi\left(A_{P}, P\right)$ on $a \in A_{P}$ is denoted $a^{\alpha}$. The differential $d \alpha$ of $\alpha$, also denoted by $\alpha$ below, is a weight of $\mathfrak{a}_{P}$ in $\mathfrak{n}_{P}$, and we have

$$
a^{\alpha}=\exp d \alpha(\log a) .
$$

There is a unique subset $\Delta\left(A_{P}, P\right)$ of $\Phi\left(A_{P}, P\right)$, consisting of $\operatorname{dim} A_{P}$ linearly independent roots, such that any element of $\Phi\left(A_{P}, P\right)$ is a linear combination with positive integral coefficients of elements of $\Delta\left(A_{P}, P\right)$, to be called the simple roots of $P$ with respect to $A_{P}$.

We recall how $\Phi\left(A_{P}, P\right)$ and $\Delta\left(A_{P}, P\right)$ are related to $\mathbb{Q}$-roots. Fix a minimal parabolic $\mathbb{Q}$-subgroup $\mathbf{P}_{0}$ and a maximal $\mathbb{Q}$-split torus $\mathbf{S}$ of $\mathbf{P}_{0}$. Let $\Phi(\mathbf{S}, \mathbf{G})$ be the set of roots of $\mathbf{G}$ with respect to $\mathbf{S}$ (the $\mathbb{Q}$-roots) and $\Delta(\mathbf{S}, \mathbf{G})$ be the set of $\mathbb{Q}$-simple roots for the ordering of $\Phi$ defined by $\mathbf{P}_{0}$. There is a unique subset $I \subset \Delta(S, G)$ such that $\mathbf{P}$ is conjugate to the standard parabolic $\mathbb{Q}$-subgroup $\mathbf{P}_{0, I}$ by a conjugation which brings the Zariski closure $\mathbf{S}_{P}$ of $A_{P}$ onto $\mathbf{S}_{I}=\left(\cap_{\alpha \in I} \text { ker } \alpha\right)^{o}$. Then, up to conjugation, the elements of $\Phi\left(A_{P}, P\right)$ are the non-zero restrictions of the elements in $\Phi^{+}(\mathbf{S}, \mathbf{G})$ and $\Delta\left(A_{P}, P\right)$ is the set of restrictions of $\Delta(\mathbf{S}, \mathbf{G})-I$.

It is known that when $\mathbf{P}$ is a minimal parabolic subgroup, there is a bijective correspondence between parabolic $\mathbb{Q}$-groups containing $\mathbf{P}$ and subsets of $\Delta\left(A_{P}, P\right)$. In fact, for any $I \subset \Delta\left(A_{P}, P\right)$, there is a unique 
parabolic $\mathbb{Q}$-subgroup $\mathbf{Q}$ denoted by $P_{I}$ such that

$$
A_{Q}=\left\{a \in A_{P} \mid a^{\alpha}=1, \alpha \in I\right\} .
$$

The same correspondence holds for any $\mathbf{P}$ which is not necessarily minimal.

For any $t>0$, define $A_{P, t}=\left\{a \in A_{P} \mid a^{\alpha}>t, \alpha \in \Delta\left(A_{P}, P\right)\right\}$. For any bounded sets $U \subset N_{P}, W \subset M_{P} K$, the subset

$$
U \times A_{P, t} \times W \subset N_{P} \times A_{P} \times\left(M_{P} K\right)=G
$$

is called a Siegel set in $G$ associated to $\mathbf{P}$ and $K$, or the basepoint $x_{0}$. For $P=G$, the Siegel sets are bounded sets. We now recall the reduction theory from $[\mathbf{B o 1}]$ in a form convenient for our applications in later sections.

\section{Proposition 2.5.}

1) There are only finitely many $\Gamma$-conjugacy classes of parabolic $\mathbb{Q}$ subgroups. Let $\mathbf{P}_{1}, \ldots, \mathbf{P}_{k}$ be a set of representatives of the $\Gamma$ conjugacy classes of parabolic $\mathbb{Q}$-subgroups. There exist Siegel sets $U_{i} \times A_{P_{i}, t_{i}} \times W_{i}$ associated to $\mathbf{P}_{i}(1 \leq i \leq k)$ whose images in $\Gamma \backslash G$ cover the whole space.

2) For any two parabolic $\mathbb{Q}$-subgroups $\mathbf{P}_{i}, i=1,2$ and Siegel sets $U_{i} \times A_{P_{i}, t_{i}} \times W_{i}$ associated to $\mathbf{P}_{i}$, the set $\left\{\gamma \in \Gamma \mid \gamma\left(U_{1} \times A_{P_{1}, t_{1}} \times\right.\right.$ $\left.\left.W_{1}\right) \cap U_{2} \times A_{P_{2}, t_{2}} \times W_{2}\right\} \neq \emptyset$ is finite.

3) Suppose that $\mathbf{P}_{1}$ is not $\Gamma$-conjugate to $\mathbf{P}_{2}$. Fix $U_{i}, W_{i}, i=1,2$. Then $\gamma\left(U_{1} \times A_{P_{1}, t_{1}} \times W_{1}\right) \cap U_{2} \times A_{P_{2}, t_{2}} \times W_{2}=\emptyset$ for all $\gamma \in \Gamma$, if $t_{1}, t_{2} \gg 0$.

4) For any fixed $U, W$, when $t \gg 0, \gamma\left(U \times A_{P, t} \times W\right) \cap U \times A_{P, t} \times W=\emptyset$ for all $\gamma \in \Gamma-\Gamma_{P}$.

5) For any two different parabolic subgroups $\mathbf{P}_{1}, \mathbf{P}_{2}$, when $t_{1}, t_{2} \gg 0$, $U_{1} \times A_{P_{1}, t_{1}} \times W_{1} \cap U_{2} \times A_{P_{2}, t_{2}} \times W_{2}=\emptyset$.

These results are not stated in exactly the same form in [Bo1] but parts (1) to (4) follow from Theorem 15.5, Proposition 15.6 and Proposition 12.6 there, and part (5) follows from part (3) and the fact that for any two different parabolic subgroups $\mathbf{P}_{1}, \mathbf{P}_{2}$, there exists an arithmetic subgroup $\Gamma$ such that $\mathbf{P}_{1}$ is not $\Gamma$-conjugate to $\mathbf{P}_{2}$. These results except part (5) are also stated in [OW, Theorem 2.11] for slightly more general discrete subgroups $\Gamma$.

Let $\Gamma \subset \mathbf{G}(\mathbb{Q})$ be an arithmetic subgroup and $\mathbf{P}$ a parabolic $\mathbb{Q}$ subgroup of $\mathbf{G}$. As usual, we let $\Gamma_{P}=\Gamma \cap P, \Gamma_{N_{P}}=\Gamma \cap N_{P}$. The groups $\Gamma_{P}$ and $\Gamma_{N_{P}}$ are arithmetic subgroups of $\mathbf{P}$ and $\mathbf{N}_{P}$ respectively and $\Gamma_{N_{P}}$ is cocompact in $N_{P}$. By [BS, Proposition 1.2], the image of $\Gamma_{P}$ in $L_{P}$ under the natural projection $P \rightarrow L_{P}=N_{P} \backslash P$ is contained in $M_{P}$ and is an arithmetic subgroup of the $\mathbb{Q}$-group $\mathbf{M}_{P}$, to be denoted by $\Gamma_{M_{P}}$. By definition, we have the exact sequence: $0 \rightarrow \Gamma_{N_{P}} \rightarrow \Gamma_{P} \rightarrow \Gamma_{M_{P}} \rightarrow 0$. 
To understand the action of $\Gamma_{P}$ on $G$ and $X$ in terms of the horospherical decomposition, we lift $\Gamma_{M_{P}}$ into $P$ by the canonical lift $i_{x_{0}}$ associated with the basepoint $x_{0}$. The image $i_{x_{0}}\left(\Gamma_{M_{P}}\right)$ is denoted by $\Gamma_{M_{P, x_{0}}}$ or $\Gamma_{M_{P}}$ below once the basepoint $x_{0}$ is fixed. Note that $i_{x_{0}}$ does not split the above exact sequence for $\Gamma_{P}$. In fact, this exact sequence does not necessarily admit a splitting.

Proposition 2.6. For every basepoint $x_{0}, \Gamma_{M_{P}, x_{0}}$ is equal to the image of $\Gamma_{P}$ in $M_{P, x_{0}}$ under the projection $P=N_{P} A_{P, x_{0}} M_{P, x_{0}} \rightarrow M_{P, x_{0}}$, and the subgroup $\Gamma_{P}$ is contained in $N_{P} \Gamma_{M_{P}, x_{0}}$.

Proof. By the definition of the canonical lift $i_{x_{0}}, \Gamma_{M_{P}, x_{0}}$ is the image of $\Gamma_{P}$ under the projection $P \rightarrow L_{P, x_{0}}$. Since $\Gamma_{N_{P}} \backslash \Gamma_{P} \subset M_{P}$, this projection image of $\Gamma_{P}$ in $L_{P, x_{0}}$ is contained in $M_{P, x_{0}}$. Hence $\Gamma_{M_{P}, x_{0}}$ is the image of $\Gamma_{P}$ under the projection $P=N_{P} A_{P, x_{0}} M_{P, x_{0}} \rightarrow M_{P, x_{0}}$.

To prove that $\Gamma_{P} \subset N_{P} \Gamma_{M_{P}, x_{0}}$, we first assume that $x_{0}$ is a rational basepoint. Then $A_{P, x_{0}}$ is a $\mathbb{Q}$-split component of $P$. By $[\mathbf{B S}$, Proposition 1.2], $\Gamma_{P} \subset N_{P} M_{P, x_{0}}$.

By Proposition 2.2, rational basepoints for $P$ always exist. Let $x_{1}$ be such a rational basepoint. By Lemma 2.1, there exists $n \in N_{P}$ such that $M_{P, x_{0}}=n M_{P, x_{1}} n^{-1}$. Since $M_{P, x_{1}}$ normalizes $N_{P}$, this implies that

$$
N_{P} M_{P, x_{0}}=N_{P} n M_{P, x_{1}} n^{-1}=N_{P} M_{P, x_{1}} \text {. }
$$

Therefore, $\Gamma_{P} \subset N_{P} M_{P, x_{1}}=N_{P} M_{P, x_{0}}$. This completes the proof of the proposition.

q.e.d.

Remark 2.7. Note that $\Gamma_{M_{P}, x_{0}}$ is in general not equal to $\Gamma \cap M_{P, x_{0}}$ even if $x_{0}$ is a rational basepoint for $P$. On the other hand, when $i_{x_{0}}$ is rational, $\Gamma_{P}$ is commensurable with both $\Gamma_{N_{P}} \Gamma_{M_{P}, x_{0}}$ and $\Gamma_{N_{P}}(\Gamma \cap$ $\left.M_{P, x_{0}}\right)$, and $\Gamma_{M_{P}, x_{0}}$ is commensurable with $\Gamma \cap M_{P, x_{0}}$. In fact, $\Gamma_{M_{P, x_{0}}} \supset$ $\Gamma \cap M_{P, x_{0}}$, and $\Gamma \cap M_{P, x_{0}}$ is a subgroup of finite index in $\Gamma_{M_{P, x_{0}}}$.

Proposition 2.8. For every basepoint $x_{0}$, we have $\Gamma_{P} \subset N_{P} \Gamma_{M_{P}, x_{0}}$, and $N_{P} \Gamma_{M_{P}, x_{0}}=N_{P} \Gamma_{P}$.

Proof. The first statement is contained in Proposition 2.6. The second statement follows from the fact in Proposition 2.6 that $\Gamma_{M_{P}, x_{0}}$ is the image of $\Gamma_{P}$ under the projection $N_{P} M_{P, x_{0}} \rightarrow M_{P, x_{0}}$. $\quad$ q.e.d.

Remark 2.9. The above proposition says that if we ignore the $N_{P}$ component, $\Gamma_{M_{P}, x_{0}}$ could be thought of as the intersection $\Gamma \cap M_{P, x_{0}}$. This proposition is crucial to the subgroup compactification in $\S 5$.

Remark 2.10. Our standing assumption in $\S 2$ is that $\mathbf{G}$ is semisimple, connected, defined over $\mathbb{Q}$. However, we want to apply some of the facts recalled or proved here to $\mathbf{M}_{P, x_{0}}$, which does not necessarily satisfy the conditions imposed on $\mathbf{G}$. We outline here the minor adjustments this requires. First, in a algebraic group $\mathbf{H}$ defined over a field of characteristic zero, a parabolic subgroup is, by definition, the normalizer of 
a parabolic subgroup of the identity component of $\mathbf{H}$. It is then also true that a parabolic subgroup is the normalizer of the unipotent radical of its identity component ([BT], 5.19).

The group $\mathbf{M}_{P}$ is reductive, but the identity component of its center $\mathbf{Z}$ is anisotropic over $\mathbb{Q}$, so that a maximal $\mathbb{Q}$-split torus is one of the derived group $\mathcal{D} \mathbf{M}_{P}$ of $\mathbf{M}_{P}$, and any rational character of $\mathbf{M}_{P}$ is trivial on $\mathbf{Z}$; hence $\mathbf{M}_{P}$ and $\mathcal{D} \mathbf{M}_{P}$ have the same system of $\mathbb{Q}$-roots.

The subgroup $M_{P, x_{0}}$ is not necessarily defined over $\mathbb{Q}$, but the projection $\pi_{P}$ of $\mathbf{P}$ onto $\mathbf{P} / \mathbf{N}_{P}=\mathbf{L}_{P}$ maps it isomorphically onto the real points of a $\mathbb{Q}$-subgroup $\mathbf{M}_{P}$. It is easily seen that a closed subgroup $H$ of $M_{P, x_{0}}$ is the intersection of $M_{P, x_{0}}$ with a parabolic $\mathbb{Q}$-subgroup of $\mathbf{P}$ if and only if its image under $\pi_{P}$ is the group of real points of a parabolic $\mathbb{Q}$-subgroup of $\mathbf{M}_{P}$. From this it is clear that the description of such subgroups, up to conjugacy in terms of subsets of simple $\mathbb{Q}$-roots of $\mathbf{L}_{P}$, is essentially the same as in 2.4.

\section{Construction of $\Gamma \backslash \bar{G}^{B S}$}

In this section, we modify the procedure in $[\mathbf{B S}]$ and use it to construct the compactification $\Gamma \backslash \bar{G}^{B S}$ in this section and $\Gamma \backslash \bar{G}^{R B S}$ in the next section. As mentioned in the introduction, the quotient $\Gamma \backslash \bar{G}^{B S} / K$ gives the Borel-Serre compactification $\Gamma \backslash \bar{X}^{B S}$.

3.1. In [Sa1], $[\mathbf{S a 2}]$ compactifications of $\Gamma \backslash X$ are constructed from compactifications of $X$ which are rational in a suitable sense. A very important point in $[\mathbf{B S}]$ is that one should start with a partial compactification of $X$ which is constructed directly from the parabolic $\mathbb{Q}$ subgroups. The procedure in $[\mathbf{B S}]$ can be summarized as follows:

1) For each parabolic $\mathbb{Q}$-subgroup $\mathbf{P}$, there is a well-defined geodesic action of $A_{P}$ on $X$, and the action gives a corner $X(P)=N_{P} \times$ $\overline{A_{P}} \times X_{P}$.

2) For any two parabolic $\mathbb{Q}$-subgroups $\mathbf{P} \subset \mathbf{Q}$, there is a canonical embedding $X(P) \hookrightarrow X(Q)$ as real analytic manifolds with corners.

3) Show that each corner $X(P)$ can be decomposed into boundary faces $e_{X}(Q)=N_{Q} \times X_{Q}$ for $\mathbf{Q} \supseteq \mathbf{P}$, i.e., $X(P)=X \cup \coprod_{\mathbf{Q} \supseteq \mathbf{P}} e_{X}(Q)$.

4) Endow $\bar{X}^{B S}=X \cup \amalg e_{X}(P)$ with the unique topology determined by the canonical topology of the corners $X(P)$.

The modified procedure in this paper is as follows:

1) For every parabolic $\mathbb{Q}$-subgroup $\mathbf{P}$ of $\mathbf{G}$, define a boundary component using the Langlands decomposition of $P$.

2) Form a partial compactification of $G$ by attaching all the rational boundary components using the horospherical coordinate decomposition with respect to $P$. 
3) Show that $\Gamma$ acts continuously on the partial compactification with a compact Hausdorff quotient, which is a compactification of $\Gamma \backslash G$.

This procedure can also be applied to $X$. Since the basic point is to start with a partial compactification of $G$ or $X$, this modified procedure is clearly similar to the original. But there are several differences. The geodesic action is avoided since there is no canonically defined geodesic action of $A_{P}$ on $G$, or equivalently the partial compactification $\bar{G}^{B S}$ depends on the choice of basepoint $x_{0}$ (see Remark 4.12). Different choices of rational boundary components give rise to different compactifications, for example, the compactification $\Gamma \backslash \bar{G}^{R B S}$ in the next section. When the boundary components are small, the partial compactification of $G$ is not a manifold with corners, and hence the first step of using corners in $[\mathbf{B S}]$ is avoided. It should be pointed out that the gluing of the boundary components at infinity is given in terms of the Langlands decomposition of parabolic subgroups and is motivated by the geodesic action.

To understand the relations between different corners $X(P)$ and the Hausdorff property of the partial compactification $\bar{X}^{B S}$, homogeneous spaces of S-type were introduced in [BS, §2]. On the other hand, these spaces can be avoided in the modified approach in this paper.

3.2. For every parabolic $\mathbb{Q}$-subgroup $\mathbf{P}$ of $\mathbf{G}$, let $P=N_{P} A_{P} M_{P}$ be the Langlands decomposition of $P=\mathbf{P}(\mathbb{R})$ with respect to the fixed basepoint $x_{0} \in X$, where $x_{0}=K \in X=G / K$. As in $\S 2$, the Langlands decomposition gives the following horospherical decomposition: $G=$ $N_{P} \times A_{P} \times\left(M_{P} K\right)$. Define the Borel-Serre boundary component $e(P)$ of $P$ by $e(P)=N_{P} \times\left(M_{P} K\right)$.

These boundary components $e(P)$ are attached at infinity of $X$ as follows. An unbounded sequence $y_{j}$ in $G$ converges to a point $\left(n_{\infty}, m_{\infty}\right) \in$ $e(P)$ if and only if in terms of the horospherical decomposition of $G$, $y_{j}=\left(n_{j}, a_{j}, m_{j}\right), n_{j} \in N_{P}, a_{j} \in A_{P}, m_{j} \in M_{P} K$, the components $n_{j}, a_{j}, m_{j}$ satisfy the conditions:

1) For any $\alpha \in \Phi\left(P, A_{P}\right),\left(a_{j}\right)^{\alpha} \rightarrow+\infty$,

2) $n_{j} \rightarrow n_{\infty}$ in $N_{P}$, and $m_{j} \rightarrow m_{\infty} \in M_{P} K$.

Boundary components are glued together as follows. For two parabolic $\mathbb{Q}$-subgroups $\mathbf{P} \subset \mathbf{Q}, \mathbf{P} \neq \mathbf{Q}, e(P)$ is attached at infinity of $e(Q)$, i.e., $e(P)$ is in the closure of $e(Q)$ in $\bar{G}^{B S}$. This gluing is defined as follows. The parabolic subgroup $\mathbf{P}$ determines a unique parabolic subgroup $\mathbf{P}^{\prime}$ of $\mathbf{M}_{Q}$. Identify $M_{Q}$ with a subgroup of $Q$ under the canonical lift $i_{x_{0}}$. Then $M_{Q} \cap P$ is the lift of $P^{\prime}$ under $i_{x_{0}}$. Similarly, under the lift $i_{x_{0}}, A_{P^{\prime}}$ and $N_{P^{\prime}}$ can be identified with subgroups of $P$. Then

$$
M_{P^{\prime}}=M_{P}, \quad A_{P}=A_{Q} A_{P^{\prime}}=A_{Q} \times A_{P^{\prime}}, N_{P}=N_{Q} N_{P^{\prime}}=N_{Q} \rtimes N_{P^{\prime}} .
$$


(see [HC, Lemma 2]). The parabolic subgroup $P^{\prime}$ induces a Langlands decomposition of $M_{Q}$ :

$$
M_{Q}=N_{P^{\prime}} \times A_{P^{\prime}} \times\left(M_{P^{\prime}} K_{Q}\right),
$$

and hence a decomposition of $e(Q)$ :

$e(Q)=N_{Q} \times\left(M_{Q} K\right)=\left(N_{Q} N_{P^{\prime}}\right) \times A_{P^{\prime}} \times\left(M_{P} K\right)=N_{P} \times A_{P^{\prime}} \times\left(M_{P} K\right)$.

Then a sequence $y_{j}$ in $e(Q)$ converges to a point $\left(n_{\infty}, m_{\infty}\right) \in e(P)$ if and only if in the decomposition

$$
y_{j}=\left(n_{j}, a_{j}^{\prime}, m_{j}\right) \in N_{P} \times A_{P^{\prime}} \times\left(M_{P} K\right)=e(Q),
$$

1) $n_{j} \rightarrow n_{\infty}, m_{j} \rightarrow m_{\infty}$

2) for all roots $\alpha \in \Phi\left(P^{\prime}, A_{P^{\prime}}\right),\left(a_{j}^{\prime}\right)^{\alpha} \rightarrow+\infty$.

The union

$$
\bar{G}^{B S}=G \cup \coprod_{\mathbf{P} \subset \mathbf{G}} e(P)
$$

with the above topology is called the Borel-Serre partial compactification of $G$. (Note that $\bar{G}^{B S}$ is not compact and hence is not a compactification of $G$.) In fact, to show that these convergent sequences define a topology, we need to show that the conditions in $[\mathbf{J M}, \S 6]$ are satisfied. Since the main condition is the double sequence condition, and this condition is satisfied by all the components in the horospherical decomposition in the above convergence, this condition is satisfied, and hence these convergent sequences define a topology.

A more traditional way to describe the topology is to give a neighborhood system. In fact, neighborhoods of boundary points can be given explicitly. For $(n, m) \in e(P)$, let $U, V$ be neighborhoods of $n, m$ in $N_{P}, M_{P} K$ respectively. As pointed out near Equation (2.6), every parabolic subgroup $\mathbf{Q}$ containing $\mathbf{P}$ corresponds to a (proper) subset $I \subset \Delta\left(P, A_{P}\right)$. Let $\mathbf{P}_{I}^{\prime}$ be the parabolic subgroup of $\mathbf{M}_{\mathbf{P}_{I}}$ defined by $\mathbf{P}$, and $A_{P_{I}^{\prime}}$ its split component of $P_{I}^{\prime}$. Then

$$
\coprod_{I \subset \Delta\left(P, A_{P}\right)} U \times A_{P_{I}^{\prime}, t} \times V
$$

is a neighborhood of $(n, m)$ in $\bar{G}^{B S}$. It can be checked that they define the same topology as one above defined by the convergent sequences. For any sequence $t_{j} \rightarrow+\infty$, and base of neighborhoods $U_{j}, V_{j}$, the above neighborhoods form a countable base for the point $(n, m)$ in $\bar{G}^{B S}$.

To understand more directly neighborhoods of the boundary points, we need to identify the closure of a Siegel set in $\bar{G}^{B S}$. For any parabolic $\mathbb{Q}$-subgroup $\mathbf{P}$, let $\Delta=\left\{\alpha_{1}, \ldots, \alpha_{r}\right\}$ be the set of simple roots in $\Phi\left(P, A_{P}\right)$. Then $A_{P}$ can be identified with $\mathbb{R}_{>0}^{r}$ under the map

$$
a \in A_{P} \rightarrow\left(a^{-\alpha_{1}}, \ldots, a^{-\alpha_{r}}\right) \in\left(\mathbb{R}_{>0}\right)^{r} \subseteq \mathbb{R}^{r} .
$$


The closure of $A_{P}$ in $\mathbb{R}^{r}$ under this embedding is denoted by $\overline{A_{P}}$.

Proposition 3.3. The embedding $N_{P} \times A_{P} \times\left(M_{P} K\right) \hookrightarrow G \subset \bar{G}^{B S}$ can be naturally extended to an embedding $N_{P} \times \overline{A_{P}} \times\left(M_{P} K\right) \hookrightarrow \bar{G}^{B S}$. The image of $N_{P} \times \overline{A_{P}} \times\left(M_{P} K\right)$ in $\bar{G}^{B S}$ is denoted by $G(P)$ and called the corner associated with $\mathbf{P}$. Furthermore, $G(P)$ is equal to $G \cup \coprod_{\mathbf{Q} \supseteq \mathbf{P}} e(Q)$.

To prove this proposition, we need to decompose $\overline{A_{P}}$ according to parabolic $\mathbb{Q}$-subgroups containing $\mathbf{P}$. Let $\mathbf{Q}$ be a parabolic $\mathbb{Q}$-subgroup containing $\mathbf{P}$. Let $\Delta\left(P, A_{P}\right)$ be the set of simple roots in $\Phi\left(P, A_{P}\right)$. As recalled in near Equation (2.6) in $\S 2$, there exists a unique subset $I$ of $\Delta\left(P, A_{P}\right)$ such that $A_{Q}=\left\{a \in A_{P} \mid a^{\alpha}=1, \alpha \in I\right\}$. Define $A_{P, Q}=\left\{a \in A_{P} \mid a^{\alpha}=1, \alpha \in \Delta \backslash I\right\}$. Then

$$
A_{P}=A_{P, Q} \times A_{Q},
$$

and this decomposition corresponds to the coordinates decomposition of $A_{P}$ under the above identification with $\left(\mathbb{R}_{>0}\right)^{r}$. It should be pointed out that this decomposition $A_{P}=A_{P, Q} \times A_{Q}$ is, in general, different from the earlier decomposition $A_{P}=A_{P^{\prime}} \times A_{Q}$ in Equation (3.1). In fact, as mentioned earlier, $A_{P^{\prime}}$ has been lifted to a subgroup of $A_{P}$ by the canonical map $i_{x_{0}}$. Then $\mathfrak{a}_{P^{\prime}}$ is orthogonal to $\mathfrak{a}_{Q}$ with respect to the Killing form, but $\mathfrak{a}_{P, Q}$ is not in general.

For each such parabolic subgroup $\mathbf{Q}$, let $o_{Q}$ be the zero point in $\overline{A_{Q}}$. From the above equation, it is clear that $A_{P, Q} \times \overline{A_{Q}} \hookrightarrow \overline{A_{P}}$; in particular, $A_{P, Q} \times o_{Q}$ is a boundary component of $\overline{A_{P}}$.

Lemma 3.4. The corner $\overline{A_{P}}$ admits a disjoint decomposition $A_{P} \cup$ $\coprod_{\mathbf{Q} \supseteq \mathbf{P}} A_{P, Q} \times o_{Q}$. In this decomposition, a sequence $a_{j} \in A_{P}$ converges to $\left(a_{\infty}, o_{Q}\right) \in A_{P, Q} \times o_{Q}$ if and only if in the decomposition $a=\left(a_{j}^{\prime}, a_{j}^{\prime \prime}\right) \in$ $A_{P, Q} \times A_{Q}, a_{j}^{\prime} \rightarrow a_{\infty}$ and $a_{j}^{\prime \prime} \rightarrow o_{Q}$ in $\overline{A_{Q}}$.

Proof. The disjoint decomposition is clear from the identification $\overline{A_{P}}=\mathbb{R}_{\geq 0}^{\Delta}$ and the one-to-one correspondence between subsets of $\Delta$ and the parabolic $\mathbb{Q}$-subgroups $Q$ containing $P$, and the convergence follows from the definition of $A_{P, Q}$.

q.e.d.

For any $\mathbf{Q} \supset \mathbf{P}$, the horospherical decomposition $G=N_{P} \times A_{P} \times$ $\left(M_{P} K\right)$ and the decomposition $A_{P}=A_{P, Q} \times A_{Q}$ give another decomposition of $G$ :

$$
G=N_{P} \times A_{P, Q} \times A_{Q} \times\left(M_{P} K\right) .
$$

This decomposition is related to the horospherical decomposition of $G$ with respect to $Q$ :

$$
G=N_{Q} \times A_{Q} \times\left(M_{Q} K\right)
$$

as shown in the following lemmas. 
Lemma 3.5. For two parabolic $\mathbb{Q}$-subgroups $\mathbf{P}, \mathbf{Q}, \mathbf{P} \subset \mathbf{Q}$, as above, let $\mathbf{P}^{\prime}$ be the unique parabolic $\mathbb{Q}$-subgroup of $\mathbf{M}_{\mathbf{Q}}$ corresponding to $\mathbf{P}$, i.e., $P^{\prime}=M_{Q} \cap P$. Then $e(Q)=N_{Q} \times\left(M_{Q} K\right)$ can be identified with $N_{P} \times A_{P^{\prime}} \times\left(M_{P} K\right)$ as in Equation (1) through the Langlands decomposition of $M_{Q}$ with respect to $P^{\prime}$. Furthermore, $e(Q) \cong N_{P} \times A_{P^{\prime}} \times\left(M_{P} K\right)$ can be identified with $N_{P} \times A_{P, Q} \times\left(M_{P} K\right)$ through the map

$$
\begin{aligned}
N_{P} \times A_{P, Q} \times\left(M_{P} K\right) & \rightarrow N_{P} \times A_{P^{\prime}} \times\left(M_{P} K\right):(n, \exp H, m) \\
& \rightarrow\left(n, \exp H_{P^{\prime}}, m\right),
\end{aligned}
$$

where $H_{P^{\prime}}$ is the component of $H$ in $\mathfrak{a}_{P^{\prime}}$ in the decomposition $\mathfrak{a}_{P}=$ $\mathfrak{a}_{P^{\prime}} \oplus \mathfrak{a}_{Q}$.

Proof. Since $N_{P}=N_{Q} N_{P^{\prime}}, A_{P}=A_{P^{\prime}} A_{Q}$, and $M_{P^{\prime}}=M_{P}$, the Langlands decomposition of $M_{Q}$ with respect to $P^{\prime}$,

$$
M_{Q}=N_{P^{\prime}} \times A_{P^{\prime}} \times M_{P^{\prime}}=N_{P^{\prime}} \times A_{P^{\prime}} \times M_{P}
$$

gives

$$
e(Q)=N_{Q} \times N_{P^{\prime}} \times A_{P^{\prime}} \times M_{P} K
$$

and hence

$$
e(Q)=N_{P} \times A_{P^{\prime}} \times M_{P} K .
$$

Since $\mathfrak{a}_{P}=\mathfrak{a}_{P, Q} \oplus \mathfrak{a}_{Q}$ and $\mathfrak{a}_{P}=\mathfrak{a}_{P^{\prime}} \oplus \mathfrak{a}_{Q}$, the map $H \in \mathfrak{a}_{P, Q} \rightarrow H_{P^{\prime}} \in \mathfrak{a}_{P^{\prime}}$ is a linear isomorphism, and the second identification is clear. q.e.d.

Lemma 3.6. In the decomposition $G=N_{P} \times A_{P, Q} \times A_{Q} \times\left(M_{P} K\right)$, a sequence $y_{j}=\left(n_{j}, a_{j}^{\prime \prime}, a_{j}, m_{j}\right)$ converges to a point in $e(Q)$ if and only if $n_{j} \rightarrow n_{\infty}, a_{j}^{\prime \prime} \rightarrow a_{\infty}^{\prime \prime}, m_{j} \rightarrow m_{\infty}$, and for $\alpha \in \Phi\left(Q, A_{Q}\right), a_{j}^{\alpha} \rightarrow+\infty$, and the limit is equal to $\left(n_{\infty}, a_{\infty}^{\prime \prime}, m_{\infty}\right) \in N_{P} \times A_{P, Q} \times\left(M_{P} K\right) \cong e(Q)$ as identified in the previous lemma.

Proof. Since the convergence to points in $e(Q)$ is defined through the horospherical decomposition of $G$ with respect to $Q$, we need to relate the above decomposition of $G$ to the horospherical decomposition associated with $Q$ :

$$
G=N_{Q} \times A_{Q} \times\left(M_{Q} K\right) .
$$

The parabolic $\mathbb{Q}$-subgroup $P^{\prime}$ of $M_{Q}$ determines a decomposition of $M_{Q} K$ :

$$
M_{Q} K=N_{P^{\prime}} \times A_{P^{\prime}} \times\left(M_{P^{\prime}} K\right)=N_{P^{\prime}} \times A_{P^{\prime}} \times\left(M_{P} K\right),
$$

which induces the following decomposition of $G$ with respect to $Q$ :

$$
G=N_{Q} \times A_{Q} \times\left(N_{P^{\prime}} \times A_{P^{\prime}} \times M_{P} K\right) .
$$

Since $N_{P^{\prime}}, A_{P^{\prime}}$ commute with $A_{Q}$ and $N_{P}=N_{Q} N_{P^{\prime}}$, we get a refined horospherical decomposition

$$
G=N_{P} \times A_{P^{\prime}} \times A_{Q} \times\left(M_{P} K\right),
$$


given by the map

$$
\left(n, a^{\prime}, a, m\right) \in N_{P} \times A_{P^{\prime}} \times A_{Q} \times\left(M_{P} K\right) \rightarrow n a^{\prime} a m \in G .
$$

We need to compare this decomposition with the earlier one

$$
G=N_{P} \times A_{P, Q} \times A_{Q} \times M_{P} K .
$$

Since the map $N_{P} \times A_{P, Q} \times A_{Q} \times M_{P} K \rightarrow G$ is given by $\left(n, a^{\prime \prime}, a, m\right) \rightarrow$ $n a^{\prime \prime} a m$ and

$$
A_{P}=A_{P^{\prime}} A_{Q}=A_{P, Q} A_{Q}
$$

in these two different decompositions, the $N_{P}, M_{P} K$ components are the same, and we only need to find relations between the $A_{P^{\prime}}, A_{Q}$ and the $A_{P, Q}, A_{Q}$ components.

Since $\mathfrak{a}_{P}=\mathfrak{a}_{P^{\prime}} \oplus \mathfrak{a}_{Q}$, for any $H \in \mathfrak{a}_{P, Q}$, write $H=H_{P^{\prime}}+H_{Q}$, where $H_{P^{\prime}} \in \mathfrak{a}_{P^{\prime}}, H_{Q} \in \mathfrak{a}_{Q}$. Then the transformation from $N_{P} \times A_{P, Q} \times A_{Q} \times$ $\left(M_{P} K\right)$ to $N_{P} \times A_{P^{\prime}} \times A_{Q} \times\left(M_{P} K\right)$ is given by

$$
(n, \exp H, \exp V, m) \mapsto\left(n, \exp H_{P^{\prime}}, \exp \left(H_{Q}+V\right), m\right) .
$$

Similarly, $\mathfrak{a}_{P}=\mathfrak{a}_{P, Q} \oplus \mathfrak{a}_{Q}$. For any $H \in \mathfrak{a}_{P^{\prime}}$, write $H=H_{P, Q}+H_{Q}$, where $H_{P, Q} \in \mathfrak{a}_{P, Q}, H_{Q} \in \mathfrak{a}_{Q}$. Then the transformation from $N_{P} \times$ $A_{P^{\prime}} \times A_{Q} \times\left(M_{P} K\right)$ to $N_{P} \times A_{P, Q} \times A_{Q} \times\left(M_{P} K\right)$ is given by

$$
(n, \exp H, \exp V, m) \mapsto\left(n, \exp H_{P, Q}, \exp \left(H_{Q}+V\right), m\right) .
$$

These two formulae of coordinate changes imply the lemma. q.e.d.

Lemma 3.7. For a pair of parabolic subgroups $\mathbf{P} \subset \mathbf{Q}$, let $I \subset$ $\Delta\left(P, A_{P}\right)$ be the subset such that $\mathfrak{a}_{Q}=\left\{H \in \mathfrak{a}_{P} \mid \alpha(H)=0, \alpha \in I\right\}$. Then under the identification

$$
e(Q) \cong N_{P} \times A_{P, Q} \times\left(M_{P} K\right)
$$

in Lemma 3.6, a sequence $y_{j}=\left(n_{j}, a_{j}, m_{j}\right)$ in e $(Q)$ converges to a point $\left(n_{\infty}, m_{\infty}\right) \in e(P)=N_{P} \times\left(M_{P} K\right)$ if and only if for all $\beta \in \Delta\left(P, A_{P}\right) \backslash I$, $a_{j}^{\beta} \rightarrow+\infty$, and $n_{j} \rightarrow n_{\infty}, m_{j} \rightarrow m_{\infty}$.

Proof. For all $H \in \mathfrak{a}_{Q}$ and $\beta \in \Delta\left(P, A_{P}\right) \backslash I, \beta(H)=0$. This implies that that for all $\beta \in \Delta\left(P, A_{P}\right) \backslash I$ and $H \in \mathfrak{a}_{P, Q}, \beta\left(H_{P^{\prime}}\right)=\beta(H)$, where $H_{P^{\prime}}$ is the component of $H$ in $\mathfrak{a}_{P^{\prime}}$ in $\mathfrak{a}_{P}=\mathfrak{a}_{P^{\prime}} \oplus \mathfrak{a}_{Q}$. Since the simple roots in $\Phi\left(P^{\prime}, A_{P^{\prime}}\right)$ are restrictions of $\Delta\left(P, A_{P}\right) \backslash I$ to $\mathfrak{a}_{P^{\prime}}$, the lemma is clear.

q.e.d.

More generally, the following lemma is true.

Lemma 3.8. Let $\mathbf{Q}_{1}, \mathbf{Q}_{2}$ be two parabolic $\mathbb{Q}$-subgroups containing $\mathbf{P}$. Suppose that $\mathbf{Q}_{1} \subset \mathbf{Q}_{2}$. Let $I_{j}$ be the subset of the simple roots in $\Delta\left(P, A_{P}\right)$ such that $\mathfrak{a}_{Q_{j}}=\left\{H \in \mathfrak{a}_{P} \mid \alpha(H)=0, \alpha \in I_{j}\right\}, j=1,2$. Under the identifications

$$
\begin{gathered}
e\left(Q_{1}\right) \cong N_{P} \times A_{P, Q_{1}} \times\left(M_{P} K\right), \\
e\left(Q_{2}\right) \cong N_{P} \times A_{P, Q_{1}} \times A_{Q_{1}, Q_{2}} \times\left(M_{P} K\right),
\end{gathered}
$$


a sequence of points $y_{j}=\left(n_{j}, a_{P, Q_{1}, j}, a_{Q_{1}, Q_{2}, j}, m_{j}\right)$ in e $\left(Q_{2}\right)$ converges in $\bar{G}^{B S}$ to a point $\left(n_{\infty}, a_{P, Q_{1}, \infty}, m_{\infty}\right) \in e\left(Q_{1}\right)$ if and only if $n_{j} \rightarrow n_{\infty}$, $a_{P, Q_{1}, j} \rightarrow a_{P, Q_{1}, \infty}, m_{j} \rightarrow m_{\infty}$, and for all $\alpha \in I_{2} \backslash I_{1},\left(a_{Q_{1}, Q_{2}, j}\right)^{\alpha} \rightarrow$ $+\infty$.

Proof of Proposition 3.3.

By Lemmas 3.5 and 3.6, the subset $N_{P} \times A_{P, Q} \times o_{Q} \times\left(M_{P} K\right)$ in $N_{P} \times$ $\overline{A_{P}} \times\left(M_{P} K\right)$ can be identified with $e(Q)$, and under this identification, convergence of sequences of interior points to points in $e(Q)$ in the topology of $N_{P} \times \overline{A_{P}} \times\left(M_{P} K\right)$ is the same as in the topology of $\bar{G}^{B S}$. By Lemma 3.7, under this identification, the convergence of sequences of points in $e(Q)$ to points in $e(P)$ in the topology of $N_{P} \times \overline{A_{P}} \times\left(M_{P} K\right)$ is the same as the convergence in the topology of $\bar{G}^{B S}$. Similarly, by Lemma 3.8, for any two boundary components $e\left(Q_{1}\right), e\left(Q_{2}\right)$ with $Q_{1} \subset$ $Q_{2}$, the convergence of sequences of points in $e\left(Q_{2}\right)$ to points in $e\left(Q_{1}\right)$ is the same in both topologies.

This implies that the embedding $N_{P} \times A_{P} \times\left(M_{P} K\right) \hookrightarrow \bar{G}^{B S}$ can be extended to an embedding

$$
N_{P} \times \overline{A_{P}} \times\left(M_{P} K\right) \hookrightarrow \bar{G}^{B S} .
$$

Lemma 3.4 and the above identification of the boundary components of $N_{P} \times \overline{A_{P}} \times\left(M_{P} K\right)$ with $e(Q)$ show that the image of $N_{P} \times \overline{A_{P}} \times\left(M_{P} K\right)$ in $\bar{G}^{B S}$ is equal to $G \cup \coprod_{Q \supseteq P} e(Q)$.

Remark 3.9. By replacing the horospherical decomposition of $G$ by the horospherical decomposition of $X$ (Equations 2.2, 2.3 in $\S 2$ ), we can apply the above procedure to construct a partial compactification of $X$, which will be shown to be isomorphic to the Borel-Serre partial compactification in [BS] (see Proposition 3.17 below).

Define

$$
\overline{A_{P}, t}=\left\{a \in \overline{A_{P}} \mid a^{\alpha}>t, \alpha \in \Delta\left(P, A_{P}\right)\right\},
$$

which is a partial compactification of $A_{P, t}$ in the direction of $P$.

Lemma 3.10. For any point $(n, m) \in N_{P} \times\left(M_{P} K\right)=e(P)$ in $\bar{G}^{B S}$, a neighborhood system of $(n, m)$ in $\bar{G}^{B S}$ is given by $U \times \overline{A_{P}, t} \times W$, where $n \in U, m \in W$ are neighborhoods in $N_{P}, M_{P} K$, and $t>0$.

Proof. Let $t$ be any large number. For any interior sequence $y_{j}$ converging to $(n, m) \in e(P)$, it follows from its definition that $y_{j} \in$ $U \times A_{P, t} \times W$ eventually. For any parabolic $\mathbb{Q}$-subgroup $\mathbf{Q} \supset \mathbf{P}$ and any sequence $y_{j}$ in $e(Q)$ converging to $(n, m) \in e(P), y_{j}$ belongs to $U \times A_{P^{\prime}, t} \times W$ eventually, where $P^{\prime}$ is the unique parabolic subgroup of $M_{Q}$ corresponding to $P$. By Lemmas 3.5, 3.6 and the proof of Lemma 
3.7, $U \times A_{P^{\prime}, t} \times W$ can be identified with $U \times\left(A_{P, Q, t} \times\left\{o_{Q}\right\}\right) \times W$ in $G(P)=N_{P} \times \overline{A_{P}} \times\left(M_{P} K\right)$, where

$$
A_{P, Q, t}=\left\{a \in A_{P, Q} \mid a^{\beta}>t, \beta \in \Delta\left(P, A_{P}\right) \backslash I\right\},
$$

$I$ being the subset of simple roots which define the subspace $\mathfrak{a}_{Q}$ in $\mathfrak{a}_{P}$. By Lemma 3.4, $\bar{A}_{P, t}=A_{P, t} \cup \coprod_{\mathbf{Q} \supseteq \mathbf{P}} A_{P, Q, t}$, this implies that every sequence in $\bar{G}^{B S}$ converging to $(n, m)$ belongs to $U \times \overline{A_{P}, t} \times W$ eventually. This shows that when $U, W$ shrink to $n, m$ respectively and $t \rightarrow+\infty$, $U \times \bar{A}_{P, t} \times W$ forms a basis of neighborhoods of $(n, m)$ in $\bar{G}^{B S}$ and hence completes the proof. $\quad$ q.e.d.

Proposition 3.11. The topology of $\bar{G}^{B S}$ is Hausdorff.

Proof. It suffices to show that any two distinct boundary points $y_{1}, y_{2}$ $\in \partial \bar{G}^{B S}$ have disjoint neighborhoods. Let $\mathbf{P}_{i}$ be the rational parabolic subgroup such that $y_{i} \in e\left(P_{i}\right)$. By Lemma 3.10, for any neighborhood $U_{i} \times W_{i}$ of $y_{i}$ in $e\left(P_{i}\right)$ and any $t>0, U_{i} \times \overline{A_{P_{i}, t}} \times W_{i}$ is a neighborhood of $y_{i}$ in $\bar{G}^{B S}$. There are two cases to consider. Suppose first that $P_{1} \neq P_{2}$. By Proposition 2.5.5, when $U_{i} \times W_{i}$ are bounded and $t \gg 0$, the Siegel sets $U_{i} \times A_{P_{i}, t} \times W_{i}, i=1,2$, are disjoint. By the proof of the previous lemma, the sets $U_{i} \times \overline{A_{P_{i}, t}} \times W_{i}$ are also disjoint, since $U_{i} \times A_{P_{i}, t} \times W_{i}$ is an open dense subset of $U_{i} \times \overline{A_{P_{i}, t}} \times W_{i}$. On the other hand, suppose that $P_{1}=P_{2}$. Then $y_{1}, y_{2}$ are two distinct points on the same boundary component $e\left(P_{1}\right)$ and hence have disjoint neighborhoods $U_{1} \times W_{1}, U_{2} \times W_{2}$. In particular, $U_{1} \times A_{P_{1}, t} \times W_{1}$ and $U_{2} \times A_{P_{2}, t} \times W_{2}$ are disjoint. This similarly implies that $U_{i} \times \overline{A_{P_{i}}} \times W_{i}$ are also disjoint.

q.e.d.

Proposition 3.12. The left $\mathbf{G}(\mathbb{Q})$-multiplication on $G$ extends to a continuous action on $\bar{G}^{B S}$. In particular, $\Gamma$ acts continuously on $\bar{G}^{B S}$ from the left.

Proof. There are two steps in the proof. The first step is to extend the left $\mathbf{G}(\mathbb{Q})$-multiplication on $G$ to $\bar{G}^{B S}$, and the second is to prove that this extended action is continuous.

For the first step, we need to show that for any $g \in \mathbf{G}(\mathbb{Q})$ and any sequence $y_{j}$ in $\bar{G}^{B S}$ converging to a boundary point $y_{\infty}$ in $\bar{G}^{B S}$, the new sequence $g y_{j}$ also converges in $\bar{G}^{B S}$ to a boundary point which depends only on $y_{\infty}$ and $g$.

Suppose that $y_{j}$ converges to $\left(n_{\infty}, m_{\infty}\right) \in e(P)$ for a parabolic $\mathbb{Q}$ subgroup $P$. We claim that $g y_{j}$ converges to a point in $e\left(g P g^{-1}\right)$.

Since $G=K P$, write $g=k m a n$, where $k \in K, n \in N_{P}, a \in A_{P}, m \in$ $M_{P}$. It is clear that $k \mathbf{P} k^{-1}=g \mathbf{P} g^{-1}$ is a rational parabolic subgroup 
also. Write $y_{j}=n_{j} a_{j} m_{j}$ as above. Then

$$
\begin{aligned}
g y_{j}=k m a n n_{j} a_{j} m_{j} & ={ }^{k m a}\left(n n_{j}\right) \cdot k m a \cdot a_{j} m_{j} \\
& ={ }^{k m a}\left(n n_{j}\right) \cdot{ }^{k}\left(a a_{j}\right) \cdot{ }^{k}\left(m m_{j}\right) \cdot k
\end{aligned}
$$

with ${ }^{k m a}\left(n n_{j}\right) \in N_{k P k^{-1}},{ }^{k}\left(a a_{j}\right) \in A_{k P k^{-1}},{ }^{k}\left(m m_{j}\right) \in M_{k P k^{-1}}$. Since $n_{j}, m_{j}$ converge, and $\left(a a_{j}\right)^{\alpha} \rightarrow+\infty$ for all $\alpha \in \Phi\left(P, A_{P}\right)$, it is then clear that $g y_{j}$ converges to the point $\left({ }^{k m a}\left(n n_{\infty}\right),{ }^{k}\left(m m_{\infty}\right) \cdot k\right) \in e\left(g P g^{-1}\right)$. We note that though the decomposition $g=k m a n$ is not unique, $k, m$ are determined by $g$ up to an element in $K \cap P=K \cap M_{P}$. This implies that the limit above does not depend on the choice of the decomposition of $g=k m a n$, and hence the sequence $g y_{j}$ has a well-defined limit.

The above discussions suggest the following definition of $\mathbf{G}(\mathbb{Q})$-action on the boundary of $\bar{G}^{B S}$ : for $g \in \mathbf{G}(\mathbb{Q})$, and $\left(n_{\infty}, m_{\infty}\right) \in e(P)$,

$$
g \circ\left(n_{\infty}, m_{\infty}\right)=\left({ }^{k m a}\left(n n_{\infty}\right),{ }^{k}\left(m m_{\infty}\right) \cdot k\right),
$$

where $g=k m a n$ as above. As showed in the previous paragraph, this action is well-defined.

To prove this extended action on $\bar{G}^{B S}$ is continuous, we need to show that for any convergent sequence $y_{j}$ in $\bar{G}^{B S}$ with limit $y_{\infty}$ and $g \in \mathbf{G}(\mathbb{Q})$, $g y_{j}$ converges to $g y_{\infty}$. The above computations prove this when $y_{j} \in G$. Therefore, it suffices to consider the case $y_{\infty} \in e(P)$ and $y_{j} \in e(Q)$ for a pair of parabolic $\mathbb{Q}$-subgroups $P \subset Q$.

Write

$$
y_{j}=\left(n_{j}, m_{j}\right) \in N_{Q} \times M_{Q} K=e(Q) .
$$

Using Equation (2) and the notation there, we can write

$$
m_{j}=\left(n_{j}^{\prime}, a_{j}^{\prime}, m_{j}^{\prime}\right) \in N_{P^{\prime}} \times A_{P^{\prime}} \times\left(M_{P} K\right) .
$$

By definition, the convergence of $y_{j}$ means that $n_{j}, n_{j}^{\prime}, m_{j}^{\prime}$ all converge with limits $n_{\infty}, n_{\infty}^{\prime}$ and $m_{\infty}^{\prime}$ respectively, and for all $\alpha \in \Phi\left(P^{\prime}, A_{P^{\prime}}\right)$, $\left(a_{j}^{\prime}\right)^{\alpha} \rightarrow+\infty$. Then the limit $y_{\infty}$ is given by

$$
y_{\infty}=\left(n_{\infty} n_{\infty}^{\prime}, m_{\infty}^{\prime}\right) .
$$

Write $g=k m a n$ where $k \in K, m \in M_{Q}, a \in A_{Q}, n \in N_{Q}$. Then

$$
g y_{j}=g \circ\left(n_{j}, m_{j}\right)=\left({ }^{k m a}\left(n n_{j}\right),{ }^{k}\left(m m_{j}\right) \cdot k\right) \in e\left(g Q g^{-1}\right) .
$$

To compute the limit of $g y_{j}$ in $\overline{e\left(k Q k^{-1}\right)}$, we decompose $m=k^{\prime} m^{\prime} a^{\prime} n^{\prime}$ where $k^{\prime} \in K \cap M_{Q}, m^{\prime} \in M_{P^{\prime}}, a^{\prime} \in A_{P^{\prime}}, n \in N_{P^{\prime}}$. By computations similar to those at the beginning of the proof, the limit of $g y_{j}$ in $\overline{e\left(k Q k^{-1}\right)}$ is equal to

$$
\left({ }^{k m a}\left(n n_{\infty}\right)^{k k^{\prime} m^{\prime} a^{\prime}}\left(n^{\prime} n_{\infty}^{\prime}\right),{ }^{k k^{\prime}}\left(m^{\prime} m_{\infty}^{\prime}\right) \cdot k k^{\prime}\right) \in e\left(g P g^{-1}\right) .
$$


By a direct computation, this limit is equal to

$$
\begin{aligned}
& \left(k k^{\prime} m^{\prime} a^{\prime}\left(n^{\prime}\right)^{k k^{\prime} m^{\prime} a^{\prime} a}\left(n n_{\infty} n_{\infty}^{\prime}\right),{ }^{k k^{\prime}}\left(m^{\prime} m_{\infty}^{\prime}\right) \cdot k k^{\prime}\right) \\
& =\left({ }^{k k^{\prime} m^{\prime} a^{\prime} a}\left(n^{\prime a} n n_{\infty} n_{\infty}^{\prime}\right),{ }^{k k^{\prime}}\left(m^{\prime} m_{\infty}^{\prime}\right) \cdot k k^{\prime}\right) .
\end{aligned}
$$

We claim that this limit is equal to $g y_{\infty}$. In fact, from $g=k m a n$ and $m=k^{\prime} m^{\prime} a^{\prime} n^{\prime}$, we obtain

$$
g=k k^{\prime} \cdot m^{\prime} \cdot a^{\prime} a \cdot n^{\prime a} n
$$

with $k k^{\prime} \in K, m^{\prime} \in M_{P}, a^{\prime} a \in A_{P}$ and $n^{\prime a} n \in N_{P}$. Then the claim follows from the equality $y_{\infty}=\left(n_{\infty} n_{\infty}^{\prime}, m_{\infty}^{\prime}\right)$ and the definition of the $\mathbf{G}(\mathbb{Q})$-action on the boundary.

q.e.d.

Proposition 3.13. The arithmetic group $\Gamma$ acts properly on $\bar{G}^{B S}$.

Proof. Since $\Gamma$ acts properly on $G$, there remains to show that a point $z$ on the boundary of $\bar{G}^{B S}$ has an open neighborhood $V$ such that $\{\gamma \in \Gamma \mid \gamma(V) \cap V \neq \emptyset\}$ is finite. By Lemma 3.10, we may assume $V=U \times \bar{A}_{P, t} \times W$, where $V^{\prime}=U \times A_{P, t} \times W$ is a Siegel set in $G$. In view of the finiteness property $2.5(2)$ of Siegel sets in $G$, it suffices to show that

$$
\gamma(V) \cap V \neq \emptyset \quad(\gamma \in \Gamma)
$$

implies

$$
\gamma\left(V^{\prime}\right) \cap V^{\prime} \neq \emptyset .
$$

Let $y$ be in the set (3.6). Since $V$ is open in $\bar{G}^{B S}$ and $\Gamma$ acts continuously (Proposition 3.12), this intersection contains an open neighborhood of $y$. The relation (3.7) now follows from the fact that $V^{\prime}$ is open dense in $V$.

q.e.d.

Theorem 3.14. The quotient $\Gamma \backslash \bar{G}^{B S}$ is a compact, Hausdorff space.

Proof. Since $\Gamma$ acts properly on $\bar{G}^{B S}$ and $\bar{G}^{B S}$ is Hausdorff, the quotient $\Gamma \backslash \bar{G}^{B S}$ is also Hausdorff. To prove that it is compact, we note that by the reduction theory (Proposition 2.5), there are finitely many parabolic $\mathbb{Q}$-subgroups $\mathbf{P}_{1}, \ldots, \mathbf{P}_{k}$ and Siegel sets $U_{1} \times A_{P_{1}, t_{1}} \times W_{1}, \ldots, U_{k} \times$ $A_{P_{k}, t_{k}} \times W_{k}$ such that the images in $\Gamma \backslash G$ cover the whole space $\Gamma \backslash G$. Clearly we can assume that $U_{i}, W_{i}$ are compact. Since the closure of $A_{P_{i}, t_{i}}$ in $\overline{A_{P_{i}}}$ is compact, by Proposition 3.3, the closure of $U_{i} \times A_{P_{i}, t_{i}} \times W_{i}$ in $\bar{G}^{B S}$ is compact. These finitely many compact subsets hence project to compact subsets and cover $\Gamma \backslash \bar{G}^{B S}$. This implies that $\Gamma \backslash \bar{G}^{B S}$ is compact.

q.e.d.

Since $\Gamma \backslash G$ is a $G$-homogeneous space, a natural question is whether the $G$-action on $\Gamma \backslash G$ extends to $\Gamma \backslash \bar{G}^{B S}$. The answer turns out to be negative as shown in the next proposition. On the other hand, we will 
show in the next two sections that $\Gamma \backslash G$ admits compactifications to which the $G$-action extends.

Proposition 3.15. The right $G$-multiplication on $\Gamma \backslash G$ does not extend to a right $G$-action on $\Gamma \backslash \bar{G}^{B S}$.

Proof. It suffices to exhibit a convergent sequence $y_{j}$ in $\Gamma \backslash \bar{G}^{B S}$ and an element $g \in G$ such that $y_{j} g$ are not convergent in $\Gamma \backslash \bar{G}^{B S}$.

Let $\mathbf{P}$ be a parabolic $\mathbb{Q}$-subgroup. Choose $H \in \mathfrak{a}_{P}$ such that for all $\alpha \in \Phi\left(P, A_{P}\right), \alpha(H)>0$. Let $y_{j}=\exp t_{j} H$ for a sequence $t_{j} \rightarrow+\infty$. Clearly, $y_{j}$ is convergent in $\bar{G}^{B S}$. Let $g=n \in N_{P}, n \neq i d$. We claim that $y_{j} g$ is not convergent in $\bar{G}^{B S}$, and its image in $\Gamma \backslash \bar{G}^{B S}$ does not converge either for suitably chosen $t_{j}$.

In fact,

$$
\begin{aligned}
y_{j} g=\left(\exp t_{j} H\right) n & =\operatorname{Int}\left(\exp t_{j} H\right)(n) \cdot \exp t_{j} H \\
& =\left(\operatorname{Int}\left(\exp t_{j} H\right)(n), \exp t_{j} H, 1\right) \in N_{P} \times A_{P} \times\left(M_{P} K\right) .
\end{aligned}
$$

The component $\operatorname{Int}\left(\exp t_{j} H\right)(n)$ in $N_{P}$ is not bounded, and hence the sequence $y_{j} g$ does not converge to any point in $\bar{G}^{B S}$. When $t_{j}$ is suitably chosen, the image of this unbounded sequence $\operatorname{Int}\left(\exp t_{j} H\right)(n)$ in $\Gamma_{N_{P}} \backslash N_{P}$ does not converge either. In fact, when $t \rightarrow+\infty$, the image of $\operatorname{Int}(\exp t H)(n)$ in $\Gamma_{N_{P}} \backslash N_{P}$ traces out a non-constant continuous path, wrapping around the "cusp" of $P$ and hence we can pick a sequence $t_{j}$ such that the image $\operatorname{Int}\left(\exp t_{j} H\right)(n)$ in $\Gamma_{N_{P}} \backslash N_{P}$ does not converge. Then the image of $y_{j} g$ in $\Gamma \backslash \bar{G}^{B S}$ does not converge to any point either. q.e.d.

\section{Remark 3.16.}

1) The above proposition can best be seen through the example of $\Gamma \backslash S L(2, \mathbb{R})$, for which the wrapping of $a n a^{-1}$, as $a \rightarrow+\infty$, around the cusps is very clear.

2 ) The proof of the above proposition shows that the right $G$-action on $G$ does not extend to a continuous action on $\bar{G}^{B S}$ either, though the right $K$-action does. This is related to the fact that these compactifications depend on the choice of the basepoint $x_{0}=K \in X$. See Remark 4.12 below. We also note that the left $\mathbf{G}(\mathbb{Q})$-multiplication on $G$ extends to $\bar{G}^{B S}$ but the right $\mathbf{G}(\mathbb{Q})$ multiplication does not, since $n$ above can be chosen in $\mathbf{N}_{P}(\mathbb{Q})$.

\section{Proposition 3.17.}

1) The right $K$-action on $G$ extends to a continuous right $K$-action on $\bar{G}^{B S}$ and $\bar{G}^{B S} / K=\bar{X}^{B S}$. 
2) The right $K$-action on $\Gamma \backslash G$ extends to a right $K$-action on $\Gamma \backslash \bar{G}^{B S}$, and the quotient of $\Gamma \backslash \bar{G}^{B S}$ by $K$ is equal to $\Gamma \backslash \bar{X}^{B S}$. Furthermore, if $\Gamma$ is neat, $\Gamma \backslash \bar{G}^{B S}$ is a $K$-principal bundle over $\Gamma \backslash \bar{X}^{B S}$.

Proof. First we need to recall briefly the definition of $\Gamma \backslash \bar{X}^{B S}$ from $[$ BS] . For any parabolic $\mathbb{Q}$-subgroup P, its induced horospherical decomposition of $X$,

$$
X=N_{P} \times A_{P} \times X_{P}, \quad X_{P}=M_{P} / K \cap M_{P},
$$

gives a geodesic action of $A_{P}$ on $X$ : for any $b \in A_{P}, x=(n, a, z) \in$ $N_{p} \times A_{P} \times X_{P}$,

$$
b \circ x=(n, a b, z) .
$$

The left multiplication of $A_{P}$ on itself clearly extends to $\overline{A_{P}}$. Then the corner $X(P)$ [BS, 5.1] associated with $\mathbf{P}$ is defined by

$$
X(P)=X \times{ }^{A_{P}} \overline{A_{P}} .
$$

For every pair of parabolic $\mathbb{Q}$-subgroups $\mathbf{P} \subset \mathbf{Q}$, it is shown in $[\mathbf{B S}, 5.3]$ that $X(Q)$ is naturally embedded into $X(P)$ as an open submanifold. For every rational parabolic subgroup $P$, define a boundary component $e_{X}(P)$ by

$$
e_{X}(P)=N_{P} \times X_{P} .
$$

Then $e_{X}(P)$ can be identified with the subset $X \times{ }^{A_{P}}\left\{o_{P}\right\}$ in $X(P)$, where $o_{P}$ is the origin of $\left(\mathbb{R}_{\geq 0}\right)^{r} \cong \overline{A_{P}}, r=\operatorname{dim} A_{P}$. The corner $X(P)$ can be written as

$$
X(P)=X \cup \coprod_{\mathbf{Q} \supseteq \mathbf{P}} e_{X}(Q) .
$$

Then the Borel-Serre partial compactification $\bar{X}^{B S}$ is defined as the disjoint union

$$
\bar{X}^{B S}=X \cup \coprod_{P} e_{X}(P)
$$

with the unique topology compatible with the topologies of all the corners $X(P)$ [BS, 7.1].

From the above description, it is clear that an unbounded sequence $y_{j}=\left(n_{j}, a_{j}, x_{j}\right) \in N_{P} \times A_{P} \times X_{P}=X$ converges to a point $\left(n_{\infty}, x_{\infty}\right) \in$ $N_{P} \times X_{P}=e_{X}(P)$ in $\bar{X}^{B S}$ if and only if the following conditions are satisfied:

1) $n_{j} \rightarrow n_{\infty}$ in $N_{P}, x_{j} \rightarrow x_{\infty}$ in $X_{P}$

2) For all $\alpha \in \Phi\left(P, A_{P}\right), a_{j}^{\alpha} \rightarrow+\infty$.

Briefly, the $A_{P}$-component needs to converge to the vertex $o_{P}$ of $\overline{A_{P}}$.

By $[\mathbf{B S}, 7.4]$, for every pair of parabolic $\mathbb{Q}$-subgroups $\mathbf{P} \subset \mathbf{Q}, \mathbf{P} \neq$ $\mathbf{Q}, e_{X}(P)$ belongs to the closure of $e_{X}(Q)$. By [BS, 7.3], convergence of points of $e_{X}(Q)$ to $e_{X}(P)$ is also given by the geodesic action on $e_{X}(Q)$ induced from $P$, where $e_{X}(Q)$ is treated as a space of type $S$ 
and $e_{X}(P)$ as a boundary component of $e_{X}(Q)$. More precisely, $\mathbf{P}$ determines a unique rational parabolic subgroup $\mathbf{P}^{\prime}$ of $\mathbf{M}_{\mathbf{Q}}$, and $\mathbf{P}^{\prime}$ gives a horospherical decomposition of $e_{X}(Q)$,

$$
e_{X}(Q)=N_{P} \times A_{P^{\prime}} \times X_{P}
$$

and the geodesic action of $A_{P^{\prime}}$ on $e_{X}(Q)$ is given by

$$
b \circ(n, a, x)=(n, a b, x) .
$$

By [BS, Proposition 7.3.(1)], sequences of points of $e_{Q}(X)$ converge to points of $e_{X}(P)$ in $\bar{X}^{B S}$ if and only if they do so in ${\overline{e_{X}(Q)}}^{B S}$. This implies that an unbounded sequence $y_{j}=\left(n_{j}, a_{j}, x_{j}\right) \in N_{P} \times A_{P^{\prime}} \times X_{P}=$ $e_{X}(Q)$ converges to $\left(n_{\infty}, x_{\infty}\right) \in N_{P} \times X_{P}=e_{X}(P)$ if and only if the following two conditions are satisfied:

1) $n_{j} \rightarrow n_{\infty}, x_{j} \rightarrow x_{\infty}$

2) For all $\alpha \in \Phi\left(P^{\prime}, A_{P^{\prime}}\right), a_{j}^{\alpha} \rightarrow+\infty$.

In summary, the convergence of sequences in $\bar{X}^{B S}$, i.e., its topology, can be described in exactly the same way as in $\bar{G}^{B S}$ given at the beginning of this section.

From the definition of $\bar{G}^{B S}$, it is clear that the right $K$-multiplication on $G$ extends to a continuous $K$-action on $\bar{G}^{B S}$ and the action is free. The description of the topologies of $\bar{G}^{B S}$ and $\bar{X}^{B S}$ implies that the $\operatorname{map} G \rightarrow X: g \rightarrow g x_{0}, x_{0}=K \in X$, extends to a continuous map $\bar{G}^{B S} \rightarrow \bar{X}^{B S}$ and the $K$-orbits are exactly the fibers of this map. This implies that the right $K$-action makes $\bar{G}^{B S}$ a principal $K$-bundle over $\bar{X}^{B S}$.

Since the right $K$-action commutes with the left $\Gamma$-action on $\bar{G}^{B S}$, dividing by $\Gamma$, we get that the right $K$-action on $\Gamma \backslash G$ extends to $\Gamma \backslash \bar{G}^{B \dot{S}}$ and its quotient by $K$ is equal to $\Gamma \backslash \bar{X}^{B S}$. When $\Gamma$ is neat, $\Gamma_{M_{P}}$ is torsion free (see [Bo1, §17]), and hence $\Gamma_{M_{P}} \cap K=i_{x_{0}}\left(\Gamma_{M_{P}}\right) \cap K$ is a torsion free finite subgroup and hence trivial, which implies that $\Gamma \backslash \bar{G}^{B S}$ is a principal bundle over $\Gamma \backslash \bar{X}^{B S}$. $\quad$ q.e.d.

Remark 3.18. For any closed subgroup $H \subset K$, the quotient of $\Gamma \backslash \bar{G}^{B S}$ by $H$ on the right gives a compactification $\Gamma \backslash \bar{G}^{B S} / H$ of $\Gamma \backslash G / H$. This remark also applies to the compactification $\Gamma \backslash \bar{G}^{R B S}$ to be constructed in the next section. If $X$ is a bounded symmetric domain and $G / H$ is a Griffiths period domain, the $\Gamma \backslash \bar{G}^{B S} / H$ compactification is the compactification $\Gamma \backslash D_{B S}$ in $[\mathbf{K U}]$, a preprint of which was received after this paper was written. 


\section{Construction of $\Gamma \backslash \bar{G}^{R B S}$}

Next we follow the general method outlined at the beginning of $\S 3$ to define the compactification $\Gamma \backslash \bar{G}^{R B S}$. An important property of this compactification is that the right $G$-action on $\Gamma \backslash G$ extends to a continuous $G$-action on $\overline{\Gamma \backslash G}^{R B S}$, which is not true for $\overline{\Gamma \backslash G}^{B S}$ (Proposition 3.15). One application of this construction is to give another definition of the reductive Borel-Serre compactification $\overline{\Gamma \backslash X}^{R B S}$ (Corollary 4.13) was first introduced by Zucker [Zu1, (4.1)].

For any parabolic $\mathbb{Q}$-subgroup $\mathbf{P}$, define its boundary component $\hat{e}(P)$ by

$$
\hat{e}(P)=M_{P} K \cong N_{P} A_{P} \backslash G .
$$

Notice that $\hat{e}(P)$ is obtained from the Borel-Serre boundary component $e(P)=N_{P} \times\left(M_{P} K\right)$ by collapsing the unipotent radical $N_{P}$, and is hence called the reductive Borel-Serre boundary component. The identification with $N_{P} A_{P} \backslash G$ shows that it is a homogeneous space of $G$.

The boundary components $\hat{e}(P)$ are attached at infinity of $G$ as follows: An unbounded sequence $y_{j}=\left(n_{j}, a_{j}, m_{j}\right) \in N_{P} \times A_{P} \times\left(M_{P} K\right)=$ $G$ converges to a point $m_{\infty} \in \hat{e}(P)$ if and only if the following two conditions are satisfied:

1) For all $\alpha \in \Phi\left(P, A_{P}\right), \quad a_{j}^{\alpha} \rightarrow+\infty$.

2) $m_{j} \rightarrow m_{\infty}$ in $M_{P} K$.

We note that unlike the case of $\bar{G}^{B S}$, there is no requirement on the

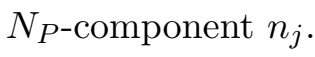

For any two parabolic $\mathbb{Q}$-subgroups $\mathbf{P} \subseteq \mathbf{Q}, \mathbf{P} \neq \mathbf{Q}$, the boundary component $\hat{e}(P)$ is attached at infinity of $\hat{e}(Q)$. As in Equation (3.1), $P$ defines a parabolic subgroup $P^{\prime}$ of $M_{Q}, P^{\prime}=P \cap M_{Q}$, satisfying $M_{P^{\prime}}=$ $M_{P}, N_{P}=N_{Q} N_{P^{\prime}}, A_{P}=A_{Q} A_{P^{\prime}}$. The group $P^{\prime}$ gives a Langlands decomposition $M_{Q}=N_{P^{\prime}} A_{P^{\prime}}\left(M_{P^{\prime}} K_{Q}\right)$ and hence a decomposition of $M_{Q} K=N_{P^{\prime}} A_{P^{\prime}} M_{P^{\prime}} K$, i.e.,

$$
\hat{e}(Q)=N_{P^{\prime}} \times A_{P^{\prime}} \times \hat{e}(P) .
$$

In this decomposition of $\hat{e}(Q)$, a sequence $y_{j}=\left(n_{j}, a_{j}, m_{j}\right)$ in $\hat{e}(Q)$ converges to a point $m_{\infty} \in \hat{e}(P)$ if and only if the following two conditions are satisfied:

1) For all $\alpha \in \Phi\left(P^{\prime}, A_{P^{\prime}}\right), a_{j}^{\alpha} \rightarrow+\infty$.

2) $m_{j} \rightarrow m_{\infty}$ in $\hat{e}(P)$.

It can be shown that these convergent sequences satisfy the conditions in $[\mathbf{J M}, \S 6]$, and hence define a topology on the disjoint union

$$
\bar{G}^{R B S}=G \cup \coprod_{P \subset G} \hat{e}(P) .
$$


In fact, the main condition to check is the double sequence condition which is satisfied since both components $a_{j}, m_{j}$ satisfy the double sequence condition.

Neighborhoods of boundary points can also be given explicitly as follows. For a parabolic $\mathbb{Q}$-subgroup $\mathbf{P}$ and a point $m \in \hat{e}(P)$, let $\mathbf{P}_{I}$, $I \subset \Delta$, be all the parabolic subgroups containing $\mathbf{P}$. For each $\mathbf{P}_{I}$, let $\mathbf{P}_{I}^{\prime}$ be the unique parabolic subgroup in $\mathbf{M}_{\mathbf{P}_{\mathbf{I}}}$ determined by $\mathbf{P}$. Let $W$ be a neighborhood of $m$ in $\hat{e}(P)=X_{P_{I}^{\prime}}=X_{P}$. Then $N_{P_{I}^{\prime}} \times A_{P_{I}^{\prime}, t} \times W$ defines a subset in $\hat{e}\left(P_{I}\right)$. The union

$$
\cup_{I} N_{P_{I}^{\prime}} \times A_{P_{I}^{\prime}, t} \times W
$$

is a neighborhood of $m$ in $\bar{G}^{R B S}$. For sequences $t_{j} \rightarrow+\infty$ and $W_{j}$ shrinking to $m$, the above sequence of neighborhoods forms a countable basis at $m$.

To show that the above topology on $\bar{G}^{R B S}$ is Hausdorff, i.e., every sequence has a unique limit, we need the following separation property of generalized Siegel sets.

\section{Proposition 4.1.}

1) For any bounded set $W \subset M_{P} K$, when $t \gg 0$, for any $\gamma \in \Gamma-\Gamma_{P}$,

$$
\gamma\left(N_{P} \times A_{P, t} \times W\right) \cap\left(N_{P} \times A_{P, t} \times W\right)=\emptyset .
$$

2) Suppose $W$ satisfies the condition that for any nontrivial $\gamma \in \Gamma_{M_{P}}$, $\gamma W \cap W=\emptyset$. Then for any $\gamma \in \Gamma-\Gamma_{N_{P}}$,

$$
\gamma\left(N_{P} \times A_{P, t} \times W\right) \cap\left(N_{P} \times A_{P, t} \times W\right)=\emptyset .
$$

3) For any two parabolic $\mathbb{Q}$-subgroups $\mathbf{P}_{1}, \mathbf{P}_{2}$ which are not conjugate under $\Gamma$, when $t \gg 0$,

$$
\gamma\left(N_{P_{1}} \times A_{P_{1}, t} \times W_{1}\right) \cap\left(N_{P_{2}} \times A_{P_{2}, t} \times W_{2}\right)=\emptyset
$$

for all $\gamma \in \Gamma$.

Proof. These separation properties are generalizations of those stated in Proposition 2.5, where the results are stated for Siegel sets $U \times A_{P, t} \times$ $W$, where $U$ is a bounded set in $N_{P}$.

Since $\Gamma_{N_{P}}$ acts cocompactly on $N_{P}$ and the condition $\gamma \in \Gamma-\Gamma_{P}$ is preserved under multiplication by elements of $\Gamma_{N_{P}}$, (1) and (3) follow immediately from Proposition 2.5.

To prove (2), we need to show that the separation holds for $\gamma \in$ $\Gamma_{P}-\Gamma_{N_{P}}$. We note that for any $\gamma \in \Gamma_{P}, \gamma\left(N_{P} \times A_{P, t} \times W\right)=N_{P} \times$ $A_{P, t} \times \gamma_{M} W$, where $\gamma_{M}$ is the image of $\gamma$ under the projection

$$
\Gamma_{P} \subset P=N_{P} \times A_{P} \times M_{P} \rightarrow \Gamma_{M_{P}} \subset M_{P} .
$$


(See Proposition 2.6.) If $\gamma \in \Gamma-\Gamma_{N_{P}}$, then $\gamma_{M}$ is nontrivial, and by the assumption on $W, \gamma_{M} W \cap W \neq \emptyset$, and hence

$\gamma\left(N_{P} \times A_{P, t} \times W\right) \cap N_{P} \times A_{P, t} \times W=N_{P} \times A_{P, t} \times \gamma_{M} W \cap N_{P} \times A_{P, t} \times W=\emptyset$.

q.e.d.

Proposition 4.2. Every convergent sequence in $\bar{G}^{R B S}$ has a unique limit, and hence the topology on $\bar{G}^{R B S}$ defined above is Hausdorff.

Proof. Since every boundary component $\hat{e}(P)$ is contained in the closure of only finitely many boundary components $\hat{e}(Q)$, it suffices to consider unbounded sequences in a fixed boundary component $\hat{e}(Q)$. Let $y_{j}$ be an unbounded sequence in $\hat{e}(Q)$ converging to a limit $y_{\infty} \in \hat{e}\left(P_{1}\right)$ for a parabolic $\mathbb{Q}$-subgroup $\mathbf{P}_{1}$ contained in $\mathbf{Q}$. Suppose $y_{j}$ converges to another limit $y_{\infty}^{\prime} \in \hat{e}\left(P_{2}\right)$, where $\mathbf{P}_{2}$ is a parabolic $\mathbb{Q}$-subgroup contained in $\mathbf{Q}$. We claim that $\mathbf{P}_{2}=\mathbf{P}_{1}$.

Denote the parabolic $\mathbb{Q}$-subgroups of $\mathbf{M}_{\mathbf{Q}}$ corresponding to $\mathbf{P}_{1}$ and $\mathbf{P}_{2}$ by $\mathbf{P}_{1}^{\prime}$ and $\mathbf{P}_{2}^{\prime}$ respectively. By definition, for any bounded neighborhood $W_{1}$ of $y_{\infty}$ in $\hat{e}\left(P_{1}\right)$ invariant on the right by $K$ and $t>0$, when $j \gg 0$,

$$
y_{j} \in N_{P_{1}^{\prime}} \times A_{P_{1}^{\prime}, t} \times W_{1} .
$$

Similarly, for such a neighborhood $W_{2}$ of $y_{\infty}^{\prime}$ in $\hat{e}\left(P_{2}\right)$, when $j \gg 0$,

$$
y_{j} \in N_{P_{2}^{\prime}} \times A_{P_{2}^{\prime}, t} \times W_{2} .
$$

If the claim is not true, i.e., $\mathbf{P}_{2}^{\prime} \neq \mathbf{P}_{1}^{\prime}$, then Proposition 4.1 , applied to $\mathbf{M}_{\mathbf{Q}}$ and the pair of parabolic subgroups $\mathbf{P}_{1}^{\prime}, \mathbf{P}_{2}^{\prime}$, shows that $N_{P_{1}^{\prime}} \times$ $A_{P_{1}^{\prime}, t} \times W_{1}$ is disjoint from $N_{P_{2}^{\prime}} \times A_{P_{2}^{\prime}, t} \times W_{2}$. This contradiction proves the claim.

Now $y_{\infty}, y_{\infty}^{\prime} \in \hat{e}\left(P_{1}\right)$. Since the coordinates of $y_{j}=\left(n_{j}, a_{j}, m_{j}\right)$ in $N_{P_{1}} \times A_{P_{1}} \times\left(M_{P_{1}} K\right)=\hat{e}(Q)$ are uniquely determined by $y_{j}, \lim _{j \rightarrow+\infty} m_{j}$ has a unique limit if it exists. This implies that $y_{\infty}=y_{\infty}^{\prime}=\lim _{j \rightarrow+\infty} m_{j}$. q.e.d.

Proposition 4.3. The left $\mathbf{G}(\mathbb{Q})$-action on $G$ extends to a continuous action on $\bar{G}^{R B S}$; in particular, $\Gamma$ acts continuously on $\bar{G}^{R B S}$.

Proof. It can be proved in exactly the same way as Proposition 3.12.

q.e.d.

Proposition 4.4. The identity map on $G$ extends to a continuous, surjective left $\mathbf{G}(\mathbb{Q})$-equivariant map $\bar{G}^{B S} \rightarrow \bar{G}^{R B S}$ which is also equivariant with respect to the right $K$-action.

Proof. For every parabolic subgroup $P$, define

$$
\pi: e(P) \rightarrow \hat{e}(P), \quad \pi((n, m))=m,
$$


where $(n, m) \in N_{P} \times\left(M_{P} K\right)=e(P)$. Extending the identity map on $G$ by the map $\pi$ on the boundary faces, we get a $\mathbf{G}(\mathbb{Q})$-equivariant surjective map $\pi: \bar{G}^{B S} \rightarrow \bar{G}^{R B S}$.

We claim that this map $\pi$ is continuous. Let $y_{j} \rightarrow y_{\infty}$ be a convergent sequence in $\bar{G}^{B S}$. We need to show that $\pi\left(y_{j}\right) \rightarrow \pi\left(y_{\infty}\right)$ in $\bar{G}^{R B S}$. It suffices to consider two cases:

1) $y_{\infty} \in e(P)$ for some $P$, and $y_{j} \in G$.

2) $y_{\infty} \in e(P)$, and $y_{j} \in e(Q)$, where $Q \supset P$.

In both cases, it is clear from the definitions of convergence of sequences in $\bar{G}^{B S}, \bar{G}^{R B S}$ that $y_{j} \rightarrow y_{\infty}$ implies $\pi\left(y_{j}\right) \rightarrow \pi\left(y_{\infty}\right)$. The right $K$-equivariance is also clear.

q.e.d.

To prove that the quotient $\Gamma \backslash \bar{G}^{R B S}$ has a Hausdorff topology, we need to identify neighborhoods of boundary points in $\bar{G}^{R B S}$.

Lemma 4.5. For every point $z \in \hat{e}(P)$, a basis of neighborhood system of $z$ in $\bar{G}^{R B S}$ is given by

$$
N_{P} \times A_{P, t} \times W \cup \coprod_{Q \supseteq P} N_{P^{\prime}} \times A_{P^{\prime}, t} \times W,
$$

where $P^{\prime}$ is the parabolic subgroup in $M_{Q}$ determined by $P, W$ is a neighborhood of $z$ in $\hat{e}(P), t>0$, and $\hat{e}(Q)$ is identified with $N_{P^{\prime}} \times A_{P^{\prime}} \times$ $\hat{e}(P)$. Furthermore, if $W$ is open, then $N_{P} \times A_{P, t} \times W \cup \coprod_{Q \supseteq P} N_{P^{\prime}} \times$ $A_{P^{\prime}, t} \times W$ is equal to $\operatorname{int}\left(\operatorname{cl}\left(N_{P} \times A_{P, t} \times W\right)\right)$, the interior of the closure of $N_{P} \times A_{P, t} \times W$ in $\bar{G}^{R B S}$. In particular, $N_{P} \times A_{P, t} \times W$ is an open dense subset of the open neighborhood $\operatorname{int}\left(\operatorname{cl}\left(N_{P} \times A_{P, t} \times W\right)\right)$ of $z$ in $\bar{G}^{R B S}$.

Proof. The first statement was mentioned earlier. In fact, for any $t>0$ and any neighborhood $W$ of $z$, if a sequence $y_{j}$ in $G$ converges to $z$ in $\bar{G}^{R B S}$, then $y_{j} \in N_{P} \times A_{P, t} \times W$. Similarly, for any $Q \supset P$, if a sequence $y_{j} \in \hat{e}(Q)$ converges to $z$ in $\bar{G}^{R B S}$, then $y_{j} \in N_{P^{\prime}} \times A_{P^{\prime}, t} \times W$. This implies that any sequence $y_{j}$ in $\bar{G}^{R B S}$ converging to $z$ belongs to $N_{P} \times A_{P, t} \times W \cup \coprod_{Q \supseteq P} N_{P^{\prime}} \times A_{P^{\prime}, t} \times W$ eventually.

To prove the second statement, we note that $\hat{e}(Q)=N_{P^{\prime}} \times A_{P^{\prime}} \times M_{P} K$ can be identified with $N_{P^{\prime}} \times A_{P, Q} \times M_{P} K$ as in Lemma 3.5. Let $\operatorname{cl}\left(N_{P} \times\right.$ $\left.A_{P, t} \times W\right)$ be the closure in $\bar{G}^{R B S}$. Then the proof of Lemma 3.6 shows that $\operatorname{cl}\left(N_{P} \times A_{P, t} \times W\right) \cap \hat{e}(Q)$ contains $N_{P^{\prime}} \times A_{P, Q, t} \times W \cong N_{P^{\prime}} \times A_{P^{\prime}, t} \times W$ as a dense open set. This proves the second statement. q.e.d.

Theorem 4.6. The quotient $\Gamma \backslash \bar{G}^{R B S}$ is a compact Hausdorff space. Proof. Let $\phi: \bar{G}^{R B S} \rightarrow \Gamma \backslash \bar{G}^{R B S}$ be the quotient map. Then the topology of $\bar{G}^{R B S}$ induces a quotient topology on $\Gamma \backslash \bar{G}^{R B S}$. 
Since $\Gamma$ does not act properly on $\bar{G}^{R B S}$, it is not automatic that the Hausdorff topology of $\bar{G}^{R B S}$ induces a Hausdorff topology on the quotient $\Gamma \backslash \bar{G}^{R B S}$.

Both the Hausdorff property and compactness of the quotient topology on $\Gamma \backslash \bar{G}^{R B S}$ follows from the reduction theory. We first prove the Hausdorff property.

For a point $z \in \hat{e}(P) \subset \bar{G}^{R B S}$, let $W$ be an open neighborhood of $z$ in $\hat{e}(P)=M_{P} K$. Let $\operatorname{int}\left(\operatorname{cl}\left(N_{P} \times A_{P, t} \times W\right)\right)$ be the interior of the closure $\operatorname{cl}\left(N_{P} \times A_{P, t} \times W\right)$ in $\bar{G}^{R B S}$, which is an open neighborhood of $z$ by Lemma 4.5. We claim that the image $\phi\left(\operatorname{int}\left(\operatorname{cl}\left(N_{P} \times A_{P, t} \times W\right)\right)\right)$ is an open neighborhood of $\phi(z)$ in $\Gamma \backslash \bar{G}^{R B S}$. In fact, the inverse image of $\phi\left(\operatorname{int}\left(\operatorname{cl}\left(N_{P} \times A_{P, t} \times W\right)\right)\right)$ in $\bar{G}^{R B S}$ is equal to

$$
\cup_{\gamma \in \Gamma} \gamma\left(\operatorname{int}\left(\operatorname{cl}\left(N_{P} \times A_{P, t} \times W\right)\right)\right),
$$

which is a union of open sets since the $\Gamma$-action is continuous, and hence open.

For two different boundary points of $\bar{G}^{R B S}$, we need to find two disjoint neighborhoods of them. For any $z \in \bar{G}^{R B S}$ and a neighborhood $U$ of $\phi(z)$ in $\Gamma \backslash \bar{G}^{R B S}$, the inverse image $\phi^{-1}(U)$ in $\bar{G}^{R B S}$ is a $\Gamma$-invariant neighborhood of $\Gamma z$. Therefore, it is equivalent to prove that for any two boundary points $z_{1}, z_{2}$ in $\bar{G}^{R B S}$ with $\Gamma z_{1} \cap \Gamma z_{2}=\emptyset$, there exist $\Gamma$-invariant neighborhoods of $\Gamma z_{1}, \Gamma z_{2}$ which are disjoint.

Let $\mathbf{P}_{1}, \mathbf{P}_{2}$ be parabolic subgroups such that $z_{1} \in \hat{e}\left(P_{1}\right), z_{2} \in \hat{e}\left(P_{2}\right)$. There are two cases to consider depending on whether $\mathbf{P}_{1}$ is $\Gamma$-conjugate to $\mathbf{P}_{2}$ or not.

In the latter case, let $W_{i}$ be a neighborhood of $z_{i}$ in $\hat{e}\left(P_{i}\right)$. By the above discussion,

$$
\cup_{\gamma \in \Gamma} \gamma \operatorname{int}\left(\operatorname{cl}\left(N_{P_{i}} \times A_{P_{i}, t} \times W_{i}\right)\right)
$$

is a $\Gamma$-invariant neighborhood of $\Gamma z_{i}, i=1,2$. We claim that when $t \gg 0$, they are disjoint. If not, there exist $\gamma_{1}, \gamma_{2} \in \Gamma$ such that

$$
\gamma_{1} \operatorname{int}\left(\operatorname{cl}\left(N_{P_{1}} \times A_{P_{1}, t} \times W_{1}\right)\right) \cap \gamma_{2} \operatorname{int}\left(\operatorname{cl}\left(N_{P_{2}} \times A_{P_{2}, t} \times W_{2}\right)\right) \neq \emptyset .
$$

Let $\gamma=\gamma_{2}^{-1} \gamma_{1}$. Then

$$
\gamma \operatorname{int}\left(\operatorname{cl}\left(N_{P_{1}} \times A_{P_{1}, t} \times W_{1}\right)\right) \cap \operatorname{int}\left(\operatorname{cl}\left(N_{P_{2}} \times A_{P_{2}, t} \times W_{2}\right)\right) \neq \emptyset .
$$

By Lemma 4.5, $\gamma \operatorname{int}\left(\operatorname{cl}\left(N_{P_{1}} \times A_{P_{1}, t} \times W_{1}\right)\right)$ and $\operatorname{int}\left(\operatorname{cl}\left(N_{P_{2}} \times A_{P_{2}, t} \times W_{2}\right)\right)$ are open in $\bar{G}^{R B S}$ and contain open dense subsets $\gamma\left(N_{P_{1}} \times A_{P_{1}, t} \times W_{1}\right)$ and $N_{P_{2}} \times A_{P_{2}, t} \times W_{2}$ respectively. It follows that the intersection

$$
\gamma \operatorname{int}\left(\operatorname{cl}\left(N_{P_{1}} \times A_{P_{1}, t} \times W_{1}\right)\right) \cap \operatorname{int}\left(\operatorname{cl}\left(N_{P_{2}} \times A_{P_{2}, t} \times W_{2}\right)\right)
$$

is open, and hence

$$
\gamma\left(N_{P_{1}} \times A_{P_{1}, t} \times W_{1}\right) \cap N_{P_{2}} \times A_{P_{2}, t} \times W_{2} \neq \emptyset .
$$


But this contradicts Proposition 4.1.(3) and hence proves the claim.

In the former case, assume that $\mathbf{P}_{1}=\mathbf{P}_{2}$ for simplicity. Choose neighborhoods $W_{1}, W_{2}$ of $z_{1}, z_{2}$ such that for all $\gamma \in \Gamma_{M_{P_{1}}}, \gamma W_{1} \cap W_{2}=$ $\emptyset$, in particular, $W_{1} \cap W_{2}=\emptyset$. Let $t \gg 0$. If $\cup_{\gamma \in \Gamma} \gamma \operatorname{int}\left(\operatorname{cl}\left(N_{P_{i}} \times A_{P_{i}, t} \times\right.\right.$ $\left.\left.W_{i}\right)\right), i=1,2$, are not disjoint, then as in the above paragraph, there exists an element $\gamma \in \Gamma$ such that

$$
\gamma\left(N_{P_{1}} \times A_{P_{1}, t} \times W_{1}\right) \cap\left(N_{P_{2}} \times A_{P_{2}, t} \times W_{2}\right) \neq \emptyset .
$$

We claim that this contradicts Proposition 4.1.(2). In fact, by Proposition 4.1.(2), this is impossible if $\gamma \notin \Gamma_{N_{P_{1}}}$. On the other hand, if $\gamma \in \Gamma_{N_{P_{1}}}$

$$
\gamma\left(N_{P_{1}} \times A_{P_{1}, t} \times W_{1}\right)=N_{P_{1}} \times A_{P_{1}, t} \times W_{1},
$$

which is disjoint from $N_{P_{2}} \times A_{P_{2}, t} \times W_{2}$ since $W_{1} \cap W_{2}=\emptyset$.

To prove the compactness of $\Gamma \backslash \bar{G}^{R B S}$, we note that for every parabolic $\mathbb{Q}$-subgroup $\mathbf{P}$ and a compact subset $U \subset N_{P}$, the closure of $U \times A_{P, t} \times M_{P} K$ in $\bar{G}^{R B S}$ is compact. This can either be seen from Lemma 4.5 or from the fact that the closure of $U \times A_{P, t} \times M_{P} K$ in $\bar{G}^{B S}$ is compact and is mapped continuously onto the closure in $\bar{G}^{R B S}$. Then the reduction theory (Proposition 2.5) implies that $\Gamma \backslash \bar{G}^{R B S}$ is covered by finitely many compact subsets and is therefore compact. q.e.d.

The boundaries of $\Gamma \backslash \bar{G}^{B S}$ and $\Gamma \backslash \bar{G}^{R B S}$ can be described as follows. Let $\mathbf{P}_{1}, \ldots, \mathbf{P}_{k}$ be representatives of $\Gamma$-conjugacy classes of proper parabolic $\mathbb{Q}$-subgroups of $\mathbf{G}$. The stabilizer in $\Gamma$ of the Borel-Serre boundary component $e\left(P_{i}\right)$, defined to be $\left\{\gamma \in \Gamma \mid \gamma\left(e\left(P_{i}\right)\right)=e\left(P_{i}\right)\right\}$, is equal to $\Gamma_{P_{i}}=\Gamma \cap P_{i}$, since $\gamma P_{i} \gamma^{-1}=P_{i}$ if and only if $\gamma \in \Gamma_{P_{i}}$. This implies

$$
\Gamma \backslash \bar{G}^{B S}=\Gamma \backslash G \cup \coprod_{i=1}^{k} \Gamma_{P_{i}} \backslash e\left(P_{i}\right)=\Gamma \backslash G \cup \coprod_{i=1}^{k} \Gamma_{P_{i}} \backslash N_{P_{i}} M_{P_{i}} K .
$$

Note that by Proposition 2.6, $\Gamma_{P_{i}} \subset N_{P_{i}} M_{P_{i}} K$. Similarly, in $\bar{G}^{R B S}$, the stabilizer of the reductive Borel-Serre boundary component $\hat{e}\left(P_{i}\right)$ is also equal to $\Gamma_{P_{i}}$. Since $\Gamma_{N_{P_{i}}}$ acts trivially on $\hat{e}\left(P_{i}\right)$, the $\Gamma_{P_{i}}$-action on $\hat{e}\left(P_{i}\right)$ factors through $\Gamma_{M_{P_{i}}}$. This implies that

$$
\Gamma \backslash \bar{G}^{R B S}=\Gamma \backslash G \cup \coprod_{i=1}^{k} \Gamma_{M_{P_{i}}} \backslash \hat{e}\left(P_{i}\right)=\Gamma \backslash G \cup \coprod_{i=1}^{k} \Gamma_{M_{P_{i}}} \backslash M_{P_{i}} K .
$$

The descriptions here suggest the following relation between $\Gamma \backslash \bar{G}^{B S}$ and $\Gamma \backslash \bar{G}^{R B S}$. 
Proposition 4.7. The identity map on $\Gamma \backslash G$ extends to a continuous map from $\Gamma \backslash \bar{G}^{B S}$ to $\Gamma \backslash \bar{G}^{R B S}$, which is equivariant for the right $K$ action. For $z \in \Gamma_{M_{P}} \backslash \hat{e}(P)$, its inverse image in $\Gamma \backslash \bar{G}^{B S}$ is equal to $\Gamma_{N_{P}} \backslash N_{P}$.

Proof. The continuous extension of the identity map on $\Gamma \backslash G$ to $\Gamma \backslash \bar{G}^{B S} \rightarrow \Gamma \backslash \bar{G}^{R B S}$ is clear from Proposition 4.4, since the map $\pi: \bar{G}^{B S} \rightarrow \bar{G}^{R B S}$ in Proposition 4.4 is continuous, left $\Gamma$-equivariant and right $K$-equivariant. Denote this extended map on $\Gamma \backslash \bar{G}^{B S}$ by $\pi$ also. Clearly, $\pi$ maps $\Gamma_{P_{i}} \backslash e\left(P_{i}\right)$ to $\Gamma_{M_{P_{i}}} \backslash \hat{e}\left(P_{i}\right)$.

To prove the second statement, we note that $e(P)$ is a bundle on $\hat{e}(P)$ with fiber equal to $N_{P}, e\left(P_{i}\right)=N_{P} \times \hat{e}\left(P_{i}\right)$, and $\pi$ projects $e\left(P_{i}\right)$ to the base $\hat{e}\left(P_{i}\right)$. Since $\Gamma_{N_{P_{i}}}, \Gamma_{M_{P_{i}}}, \Gamma_{P_{i}}$ fit into an exact sequence, $0 \rightarrow \Gamma_{N_{P_{i}}} \rightarrow \Gamma_{P_{i}} \rightarrow \Gamma_{M_{P_{i}}} \rightarrow 0$, and $\Gamma_{M_{P_{i}}}=\Gamma_{M_{P_{i}}, x_{0}}$ is the image of $\Gamma_{P_{i}}$ under the projection $N_{P_{i}} M_{P_{i}} \rightarrow M_{P_{i}}$ (Proposition 2.6), it follows that the boundary component $\Gamma_{P} \backslash e(P)$ in $\Gamma \backslash \bar{G}^{B S}$ is a bundle over the boundary component $\Gamma_{M_{P}} \backslash \hat{e}(P)$ in $\Gamma \backslash \bar{G}^{R B S}$ with fiber equal to $\Gamma_{N_{P}} \backslash N_{P}$, and the map from $\Gamma_{P} \backslash e(P)$ to $\Gamma_{M_{P}} \backslash \hat{e}(P)$ is the projection to the base. This implies that the inverse image in $\Gamma \backslash \bar{G}^{B S}$ of a boundary point in $\Gamma_{M_{P}} \backslash \hat{e}(P)$ is equal to $\Gamma_{N_{P}} \backslash N_{P}$.

q.e.d.

Proposition 4.8. The right $G$-multiplication on $G$ extends to a continuous action on $\bar{G}^{R B S}$, and hence the right $G$-multiplication on $\Gamma \backslash G$ extends to a continuous $G$-action on $\Gamma \backslash \bar{G}^{R B S}$.

Proof. To prove that the right $G$-multiplication extends to a continuous action on $\bar{G}^{R B S}$, we first show that if $y_{j}$ is an unbounded sequence in $G$ converging in $\bar{G}^{R B S}$, then for any $g \in G, y_{j} g$ is also convergent in $\bar{G}^{R B S}$. This will motivate a definition of the right $G$-action on $\Gamma \backslash \bar{G}^{R B S}$.

Suppose $y_{j}$ converges to a point $m_{\infty} \in \hat{e}(P)$. Write $y_{j}=\left(n_{j}, a_{j}, m_{j}\right) \in$ $N_{P} \times A_{P} \times\left(M_{P} K\right)$. Then $m_{j} \rightarrow m_{\infty}$ in $M_{P} K$, and for all $\alpha \in$ $\Phi\left(P, A_{P}\right),\left(a_{j}\right)^{\alpha} \rightarrow+\infty$, but there is no condition on $n_{j}$.

Write

$$
m_{j} g=n_{j}^{\prime} a_{j}^{\prime} m_{j}^{\prime}=\left(n_{j}^{\prime}, a_{j}^{\prime}, m_{j}^{\prime}\right) \in N_{P} \times A_{P} \times\left(M_{P} K\right) .
$$

Since $m_{j} g \rightarrow m_{\infty} g$, the components $n_{j}^{\prime}, a_{j}^{\prime}, m_{j}^{\prime}$ all converge. Let $m_{\infty}^{\prime}=$ $\lim _{j \rightarrow \infty} m_{j}^{\prime}$. Now

$$
\begin{aligned}
y_{j} g=n_{j} a_{j} m_{j} g=n_{j} a_{j} n_{j}^{\prime} a_{j}^{\prime} m_{j}^{\prime} & =n_{j} a_{j} n_{j}^{\prime} a_{j}^{-1} a_{j} a_{j}^{\prime} m_{j}^{\prime} \\
& =\left(n_{j} a_{j} n_{j}^{\prime} a_{j}^{-1}, a_{j} a_{j}^{\prime}, m_{j}^{\prime}\right) .
\end{aligned}
$$

For all $\alpha \in \Phi\left(P, A_{P}\right),\left(a_{j} a_{j}^{\prime}\right)^{\alpha}=\left(a_{j}\right)^{\alpha}\left(a_{j}^{\prime}\right)^{\alpha} \rightarrow+\infty$, since $a_{j}^{\prime}$ is bounded. This implies that $y_{j} g$ converges in $\bar{G}^{R B S}$ to $m_{\infty}^{\prime} \in \hat{e}(P)$. 


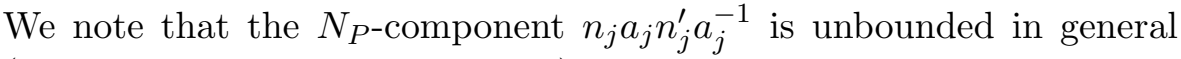
(see the proof of Proposition 3.15), but this does not affect the convergence in $\bar{G}^{R B S}$, since there is no condition on the $N_{P \text {-component for }}$ the convergent sequences.

We note that the limit $m_{\infty}^{\prime}=\lim _{j \rightarrow+\infty} m_{j}^{\prime}$ is equal to the $M_{P} K$ component of $m_{\infty} g$ in the decomposition $G=N_{P} \times A_{P} \times M_{P} K$, and hence only depends on $m_{\infty}$ and $g$ but not on the choice of the sequence $y_{j}$ converging to $m_{\infty}$.

The above computation and remark suggest the following right $G$ action on the boundary of $\bar{G}^{R B S}$. For any boundary point $m \in \hat{e}(P)=$ $M_{P} K$, and an element $g \in G$, write $m g=\left(n^{\prime}, a^{\prime}, m^{\prime}\right) \in N_{P} \times A_{P} \times M_{P} K$. Then define

$$
m \cdot g=m^{\prime} \in \hat{e}(P) .
$$

Combined with the right multiplication on $G$, this gives a right action of $G$ on $\bar{G}^{R B S}$. When $\hat{e}(P)=M_{P} K$ is identified with $N_{P} A_{P} \backslash G$, this action of $G$ is given by the right multiplication. Then it is clear that each boundary component $\hat{e}(P)$ is preserved by the $G$-action and acted upon transitively by $G$, and the decomposition

$$
\bar{G}^{R B S}=G \cup \coprod_{P} \hat{e}(P)
$$

is the orbit decomposition of $\bar{G}^{R B S}$ under this $G$-action.

The above computations show that for any unbounded sequence $y_{j} \in$ $G$ converging to $m_{\infty} \in \hat{e}(P)$ in $\bar{G}^{R B S}$ and any $g \in G, y_{j} g$ converges to $m_{\infty} g$. It can be proved similarly that if $g_{j} \rightarrow g$ in $G$, then $y_{j} g_{j}$ also converges to $m_{\infty} g$. In fact, in the decomposition $m_{j} g_{j}=\left(n_{j}^{\prime}, a_{j}^{\prime}, m_{j}^{\prime}\right)$ as above, $\lim _{j \rightarrow \infty} m_{j}^{\prime}$ exists and depends only on $m_{\infty}$ and $g$, and the rest of the arguments goes through.

We can show similarly that the same conclusion holds when $y_{j}$ is a sequence of points in $\hat{e}(Q)$ converging to $m_{\infty} \in \hat{e}(P)$ in $\bar{G}^{R B S}$, where $Q \supset P$. This shows that the right $G$-action on $\bar{G}^{R B S}$ is continuous.

The second statement in the proposition follows from the first one since the left $\Gamma$-action on $\bar{G}^{R B S}$ commutes with the right $G$-action. q.e.d.

Proposition 4.9. The right $G$-action on $\Gamma \backslash \bar{G}^{R B S}$ has finitely many orbits, and the decomposition into the $G$-orbits is the same as the decomposition into boundary components:

$$
\Gamma \backslash \bar{G}^{R B S}=\Gamma \backslash G \cup \coprod_{i=1}^{k} \Gamma_{M_{P_{i}}} \backslash M_{P_{i}} K=\Gamma \backslash G \cup \coprod_{i=1}^{k} \Gamma_{M_{P_{i}}} N_{P_{i}} A_{P_{i}} \backslash G,
$$

where $\mathbf{P}_{1}, \ldots, \mathbf{P}_{k}$ are representatives of $\Gamma$-conjugacy classes of proper parabolic $\mathbb{Q}$-subgroups. 
Proof. The definition of the right action of $G$ on the boundary of $\bar{G}^{R B S}$ in the proof of the previous proposition shows that $G$ preserves and acts transitively on each boundary component $\hat{e}(P)$. This implies that the $G$-action on $\Gamma \backslash \bar{G}^{R B S}$ preserves each boundary component $\Gamma_{M_{P_{i}}} \backslash M_{P_{i}} K$ and acts transitively on it.

q.e.d.

Remark 4.10. The proof of the above proposition shows that the real locus $G$ acts on $\bar{G}^{R B S}$ from the right and leaves stable all the rational boundary faces. On the other hand, the left $\mathbf{G}(\mathbb{Q})$-action on $G$ maps the Langlands decomposition of one parabolic subgroup to another, and hence maps one rational boundary face of $\bar{G}^{R B S}$ to another boundary component (see the proof of Proposition 3.12). This difference between the left and the right actions is caused by viewing $G$, in this paper, as a right $K$-principal bundle over $X=G / K$. The left $K$-action on $G$ induces the left $K$-action on $X=G / K$ and permutes all (real) parabolic subgroups of $G$. On the other hand, the right $K$-action on $G$ gives a trivial action on $X$ and hence leaves all the boundary faces of $\bar{G}^{R B S}$ stable.

Proposition 4.11. The compactifications $\bar{G}^{R B S}$ and $\Gamma \backslash \bar{G}^{R B S}$ are independent of the choice of basepoint $x_{0}=K \in X$.

Proof. Let $x_{1}$ be any basepoint different from the fixed basepoint $x_{0}$. Let the compactifications $\bar{G}^{R B S}$ defined with respect to them be denoted by $\bar{G}_{x_{1}}^{R B S}$ and $\bar{G}_{x_{0}}^{R B S}$. For any parabolic $\mathbb{Q}$-subgroup $P$, assume that $y_{j}$ is a sequence in $G$ converging to a point $m_{\infty} \in \hat{e}(P)$ in $\bar{G}_{x_{0}}^{R B S}$. Then, by definition,

$$
y_{j}=\left(n_{j}, a_{j}, m_{j}\right) \in N_{P} \times A_{P, x_{0}} \times M_{P, x_{0}} K
$$

with the components satisfying the following conditions: (1) $m_{j} \rightarrow m_{\infty}$, (2) for all $\alpha \in \Phi\left(P, A_{P, x_{0}}\right),\left(a_{j}\right)^{\alpha} \rightarrow+\infty$.

Write $x_{1}=p_{0} x_{0}$ and $m_{\infty} p_{0}=\left(n_{1}, a_{1}, m_{1}\right) \in N_{P} \times A_{P, x_{0}} \times M_{P, x_{0}} K$ as in Lemma 2.3. In the horospherical decomposition with respect to the basepoint $x_{1}$,

$$
y_{j}=\left(n_{j}^{\prime}, a_{j}^{\prime}, m_{j}^{\prime}\right) \in N_{P} \times A_{P, x_{1}} \times p_{0} M_{P, x_{0}} K p_{0}^{-1},
$$

it follows from Lemma 2.3.(2) that (1) $m_{j}^{\prime} \rightarrow p_{0} m_{0}^{-1} m_{1} p_{0}^{-1},(2)$ for all $\alpha^{\prime} \in \Phi\left(P^{\prime}, A_{P^{\prime}}\right),\left(a_{j}^{\prime}\right)^{\alpha^{\prime}} \rightarrow+\infty$.

Therefore, $y_{j}$ converges to the point $p_{0} m_{0}^{-1} m_{1} p_{0}^{-1} \in \hat{e}(P)$ in $\bar{G}_{x_{1}}^{R B S}$. This implies that the identity map on $G$ extends to a continuous map from $\bar{G}_{x_{0}}^{R B S}$ to $\bar{G}_{x_{1}}^{R B S}$. By changing the role of $x_{0}$ and $x_{1}$, it is clear that this extended map is an isomorphism, and hence the compactification $\bar{G}_{x_{0}}^{R B S}$ is independent of the choice of the basepoint $x_{0}$. Since $\Gamma \backslash \bar{G}^{R B S}$ 
is a quotient of $\bar{G}^{R B S}$ by $\Gamma$, it is also independent of the choice of the basepoint.

q.e.d.

Remark 4.12. It is shown in $[\mathbf{B S}]$ (and can also be deduced from 2.3(1) here), that $\bar{X}^{B S}$ and $\Gamma \backslash \bar{X}^{B S}$ are independent of the choice of a base point. It is then also true for $\bar{X}^{R B S}$ and $\Gamma \backslash \bar{X}^{R B S}$. On the other hand, the compactifications $\bar{G}^{B S}$ and $\Gamma \backslash \bar{G}^{B S}$ do depend on the choice of the basepoint $x_{0}$, i.e., for two different basepoints $x_{0}, x_{1}$, the identity map on $G$ does not necessarily extend to a homeomorphism $\bar{G}_{x_{0}}^{B S} \rightarrow \bar{G}_{x_{1}}^{B S}$. In the above proof, assume that $y_{j}=\left(n_{j}, a_{j}, m_{j}\right) \in$ $N_{P} \times A_{P, x_{0}} \times M_{P, x_{0}} K$ converges to a limit $\left(n_{\infty}, m_{\infty}\right) \in e(P)$ in $\bar{G}^{B S}$ with respect to the basepoint $x_{0}$. For simplicity, assume that $n_{j}=$ $n, m_{j}=m$ for some $n, m$. Write $m p_{0}=\left(n_{1}, a_{1}, m_{1}\right)$ as above. By Lemma 2.3.(2), the $N_{P}$-component of $y_{j}$ with respect to the basepoint $x$ is $n\left(a_{j} n_{1} a_{j}^{-1}\right) n_{0}^{-1}$. For a generic basepoint $x=p_{0} x_{0}, n_{1}$ is nontrivial. Then $a_{j} n_{1} a_{j}^{-1}$ is not bounded as $j \rightarrow+\infty$. This implies that $y_{j}$ is not convergent in $\bar{G}^{B S}$ with respect to the basepoint $x$. This is similar to the computation in the proof of Proposition 3.15, and hence shows that the dependence of $\bar{G}^{B S}$ on the basepoint is equivalent to the fact the right $G$-multiplication on $G$ does not extend to the compactification $\bar{G}^{B S}$. It is also clear from the proof of Propositions 4.8 and 4.11 that the independence of $\bar{G}^{R B S}$ on the choice of the basepoint is equivalent to the continuous right $G$-action on $\bar{G}^{R B S}$. A natural question is whether there is a canonical homeomorphism from $\bar{G}_{x_{0}}^{B S} \rightarrow \bar{G}_{x_{1}}^{B S}$ which is determined by the basepoints $x_{0}, x_{1}$ and does not necessarily restrict to the identity on $G$. When $x_{1}$ is rational with respect to $x_{0}$, i.e., $x_{1}=g x_{0}$ for some $g \in \mathbf{G}(\mathbb{Q})$, conjugation by $g$ gives such a homeomorphism. Otherwise, it is not clear how to construct such a homeomorphism.

As an application of the compactification $\Gamma \backslash \bar{G}^{R B S}$ in this section, we can recover the reductive Borel-Serre compactification $\Gamma \backslash \bar{X}^{R B S}$. In [Zu1, (4.1)], Zucker defined the reductive Borel-Serre compactification $\overline{\Gamma \backslash X}^{R B S}$ by collapsing certain nilmanifolds in the boundary of the BorelSerre compactification $\overline{\Gamma \backslash X}^{B S}$. More precisely, let $\mathbf{P}_{1}, \ldots, \mathbf{P}_{k}$ be representatives of $\Gamma$-conjugate classes of proper parabolic $\mathbb{Q}$-subgroups. Then

$$
\Gamma \backslash \bar{X}^{B S}=\Gamma \backslash X \cup \coprod_{i=1}^{k} \Gamma_{P_{i}} \backslash\left(N_{P_{i}} \times X_{P_{i}}\right)=\Gamma \backslash X \cup \coprod_{i=1}^{k} \Gamma_{P_{i}} \backslash N_{P_{i}} M_{P_{i}} / K_{P_{i}},
$$

where $X_{P_{i}}=M_{P_{i}} / K_{P_{i}}, K_{P_{i}}=K \cap M_{P_{i}}$. Each boundary component $\Gamma_{P_{i}} \backslash N_{P_{i}} M_{P_{i}} / K_{P_{i}}$ is a bundle over $\Gamma_{M_{P_{i}}} \backslash M_{P_{i}} / K_{P_{i}}$ with fiber equal to $\Gamma_{N_{P_{i}}} \backslash N_{P_{i}}$, which is a nilmanifold, i.e., quotient of a nilpotent group. 
(See the proof of Proposition 3.17 for more details on $\bar{X}^{B S}$ and hence $\Gamma \backslash \bar{X}^{B S}$.) Collapsing these fibers $\Gamma_{N_{P_{i}}} \backslash N_{P_{i}}$ in the boundary of $\Gamma \backslash \bar{X}^{B S}$ gives the reductive Borel-Serre compactification $\Gamma \backslash \bar{X}^{R B S}$ :

$$
\begin{aligned}
\Gamma \backslash \bar{X}^{R B S} & =\Gamma \backslash X \cup \coprod_{i=1}^{k} \Gamma_{M_{P_{i}}} \backslash M_{P_{i}} / K_{P_{i}} \\
& =\Gamma \backslash X \cup \coprod_{i=1}^{k} \Gamma_{M_{P_{i}}} \backslash X_{P_{i}} .
\end{aligned}
$$

This compactification $\Gamma \backslash \bar{X}^{R B S}$ also plays a crucial role in the theory of weighted cohomology in $[\mathbf{G H M}]$. A corollary of Theorem 4.6 gives another construction of $\Gamma \backslash \bar{X}^{R B S}$.

Corollary 4.13. The quotient of $\Gamma \backslash \bar{G}^{R B S}$ by $K$ on the right is a compactification of $\Gamma \backslash X$ equal to the reductive Borel-Serre compactification $\overline{\Gamma \backslash X}^{R B S}$. Furthermore, if $\Gamma$ is neat, the former is a K-principal bundle over the latter.

Proof. Let $\pi: X \rightarrow \Gamma \backslash X$ be the projection map. For any parabolic $\mathbb{Q}$-subgroup $\mathbf{P}$ and a sequence $y_{j}=\left(n_{j}, a_{j}, x_{j}\right) \in N_{P} \times A_{P} \times X_{P}=X$, if $x_{j} \rightarrow x_{\infty}$ in $X_{P}, n_{j} \rightarrow n_{\infty}$ in $N_{P}$, and for all $\alpha \in \Phi\left(P, A_{P}\right),\left(a_{j}\right)^{\alpha} \rightarrow$ $+\infty$, then $y_{j}$ converges to $\left(n_{\infty}, x_{\infty}\right) \in N_{P} \times X_{P}=e_{X}(P)$ in the BorelSerre partial compactification $\bar{X}^{B S}$ (see the proof of Proposition 3.17). Hence the sequence $\pi\left(y_{j}\right)$ in $\Gamma \backslash X$ converges in $\Gamma \backslash \bar{X}^{B S}$. By the definition of $\Gamma \backslash \bar{X}^{R B S}$ in $[\mathbf{Z u 1},(4.1)]$ the image $\pi\left(y_{j}\right)$ in $\Gamma \backslash X$ also converges in $\Gamma \backslash \bar{X}^{R B S}$ to the image of $x_{\infty}$ in $\Gamma_{M_{P}} \backslash X_{P}$, and the limit is independent of $n_{\infty}$. This implies that for any sequence $y_{j}$ in $X$ with $a_{j}, x_{j}$ satisfying the same condition as above but $n_{j}$ being bounded, the image $\pi\left(y_{j}\right)$ also converges in $\Gamma \backslash \bar{X}^{R B S}$ to the image of $x_{\infty}$ in $\Gamma_{M_{P}} \backslash X_{P}$.

Since any sequence $g_{j}$ in $\Gamma \backslash G$ converging in $\Gamma \backslash \bar{G}^{R B S}$ to a boundary point in $\Gamma_{M_{P}} \backslash \hat{e}(P)$ has a lift $\tilde{g}_{j}$ in $\Gamma \backslash G$ such that the components in $\tilde{g}_{j}=\left(n_{j}, a_{j}, m_{j}\right) \in N_{P} \times A_{P} \times M_{P} K$ satisfy : (1) $n_{j}$ is bounded, (2) $m_{j} \rightarrow m_{\infty}$, and (3) for all $\alpha \in \Phi\left(P, A_{P}\right),\left(a_{j}\right)^{\alpha} \rightarrow+\infty$, by the previous paragraph, the sequence $\pi\left(g x_{0}\right)=\pi\left(\tilde{g} x_{0}\right)$ in $\Gamma \backslash X$ converges in $\Gamma \backslash \bar{X}^{R B S}$ to the image of $m_{\infty} K_{P}$ in $\Gamma_{M_{P}} \backslash X_{P}$, where $x_{0}=K \in X$. Clearly, the limit only depends on the orbit of $m_{\infty} K$ in $M_{P} K$. This implies that there is a continuous map from $\Gamma \backslash \bar{G}^{R B S} / K$ to $\Gamma \backslash \bar{X}^{R B S}$ which extends the identity map in the interior $\Gamma \backslash G / K \rightarrow \Gamma \backslash X$, and maps the quotient by $K$ of each boundary component $\Gamma_{M_{P_{i}}} \backslash \hat{e}\left(P_{i}\right)=\Gamma_{M_{P_{i}}} \backslash M_{P_{i}} K$ onto $\Gamma_{M_{P_{i}}} \backslash M_{P_{i}} / K_{P_{i}}=\Gamma_{M_{P_{i}}} \backslash X_{P_{i}}$, a boundary component of $\Gamma \backslash \bar{X}^{R B S}$. Clearly, this map is bijective and hence is a homeomorphism. When $\Gamma$ 
is neat, $\Gamma_{M_{P_{i}}}$ is torsion free, and the $K$-action on the boundary components $\Gamma_{M_{P_{i}}} \backslash M_{P_{i}} K$ and hence on $\Gamma \backslash \bar{G}^{R B S}$ is free. This implies that $\Gamma \backslash \bar{G}^{R B S}$ is a $K$-principal bundle over $\Gamma \backslash \bar{X}^{R B S}$. $\quad$ q.e.d.

Proposition 4.14. Let $\sigma: K \rightarrow G L(n, \mathbb{C})$ be a finite dimensional representation of $K$ and $E_{\sigma}=\Gamma \backslash G \otimes_{K} \mathbb{C}^{n}$ the associated locally homogeneous bundle on $\Gamma \backslash X$. Then the bundle $E_{\sigma}$ extends to $\Gamma \backslash \bar{X}^{B S}$ and $\Gamma \backslash \bar{X}^{R B S}$.

Proof. Since $\Gamma \backslash \bar{G}^{B S} / K=\Gamma \backslash \bar{X}^{B S}, \Gamma \backslash \bar{G}^{B S} \otimes_{K} \mathbb{C}^{n}$ is the desired extension over $\Gamma \backslash \bar{X}^{B S}$. The extension over $\Gamma \backslash \bar{X}^{R B S}$ is similarly obtained. q.e.d.

Remark 4.15. We can also define $\Gamma \backslash \bar{X}^{R B S}$ more directly using the same procedure as in $\S 4$ without using $\Gamma \backslash \bar{G}^{R B S}$. More precisely, for each parabolic $\mathbb{Q}$-subgroup $\mathbf{P}$, choose the boundary component $\hat{e}(P)=$ $X_{P}$, and apply the method in $\S 3$ to construct a partial compactification $\bar{X}^{R B S}$, whose quotient by $\Gamma$ is $\Gamma \backslash \bar{X}^{R B S}$. The point of the construction in this section is to obtain $\Gamma \backslash \bar{X}^{R B S}$ independently of $\Gamma \backslash \bar{X}^{B S}$.

\section{Realization of $\Gamma \backslash \bar{G}^{R B S}$ in the space of closed subgroups}

So far, $\Gamma$ was always a subgroup of $\mathbf{G}(\mathbb{Q})$. In this section, we shall call, slightly more generally, arithmetic a discrete subgroup of $G$ (i.e. $\mathbf{G}(\mathbb{R})$ ) which is commensurable with an arithmetic subgroup of $\mathbf{G}(\mathbb{Q})$. In particular "maximal discrete" will be meant as a subgroup of $G$. Note that if $G$ is of adjoint type, there is no such distinction since any subgroup commensurable with an arithmetic one is automatically contained in $\mathbf{G}(\mathbb{Q})[$ Bo3]. We assume in this section that all normal $\mathbb{Q}$ subgroups of $\mathbf{G}$ have strictly positive $\mathbb{Q}$-rank. Then $G$ has no compact factor of strictly positive dimension.

In the previous two sections, we have followed the procedure suggested by [BS] and constructed $\Gamma \backslash \bar{G}^{B S}, \Gamma \backslash \bar{G}^{R B S}$ by defining directly boundary points and convergence of interior points to them. Another approach to compactify $X$ (or any homogeneous space of $G$ ) is to embed $X$ into a compact space $Z$ and take the closure. If moreover $Z$ is a $G$-space and the embedding is $G$-equivariant, the compactification thus obtained is automatically a $G$-space. In that case, one has to analyze the $G$-structure of the boundary and the convergence of interior points to it. This procedure was initiated by Satake [Sa1] and slightly later by Furstenberg $[\mathbf{F u}]$ for the symmetric space $X=G / K$, and then the compactifications of $X$ were applied by Satake and others to $\Gamma \backslash X$ ([Sa2], $[\mathbf{B B}])$. In this section, we show that if $\Gamma$ is arithmetic and maximal, 
$\Gamma \backslash \bar{G}^{R B S}$ can be defined in this way, using for $Z$ the space $\mathcal{S}(G)$ of closed subgroups of $G$.

Since $G$ has no compact factor of positive dimension, it is shown in $[\mathbf{K M}]$ (see [Bo4]) that the volume of $\Gamma \backslash G$ has a strictly positive lower bound. As a consequence any discrete subgroup of finite volume of $G$ is contained in a maximal discrete one (not necessarily unique). Moreover, we have the following lemma.

Lemma 5.1. Let $\Gamma \subset G$ be discrete, of finite covolume. Then the normalizer $\mathcal{N}(\Gamma)$ in $G$ is discrete.

Proof. Let $M$ be the (ordinary) closure of $\mathcal{N}(\Gamma)$. It is the real locus of an algebraic subgroup $\mathbf{M}$ of $\mathbf{G}$. Its identity component $M^{0}$ centralizes $\Gamma$. But $\Gamma$ is Zariski dense in $G$ [Bo2], hence $M^{0}$ is reduced to the identity, and hence $M=\mathcal{N}(\Gamma)$ is discrete.

q.e.d.

In particular we see that $\Gamma$ is of finite index in $\mathcal{N}(\Gamma)$ and if $\Gamma$ is maximal discrete, then $\Gamma=\mathcal{N}(\Gamma)$. Of course, $\Gamma$ may be equal to its normalizer without being maximal.

Examples of maximal arithmetic subgroups are given in [Bo3] and $[\mathbf{A}]$. In particular, $S L_{n}(\mathbb{Z})$ and $S p_{2 n}(\mathbb{Z})$ are maximal in $S L_{n}(\mathbb{R})$ and $S p_{2 n}(\mathbb{R})$ respectively. More generally, if $\mathbf{G}$ is split over $\mathbb{Q}$, then an arithmetic subgroup associated to an admissible Chevalley lattice is maximal ([Bo3, Theorem 7]). Such examples can also be defined in a split $k$ group if $k$ is a number field with class number one (loc.cit.). For other examples, see $[\mathbf{A}]$.

Let $\mathcal{S}(G)$ be the space of closed subgroups of $G$. We first recall several basic facts about $\mathcal{S}(G)$ from [Bu, Chap. 8, §5], which also hold when $G$ is replaced by any locally compact separable group.

Proposition 5.2. The space $\mathcal{S}(G)$ may be endowed with a topology under which it is a compact, Hausdorff $G$-space, $G$ acting by conjugation.

Two definitions of the topology are given in $[\mathbf{B u}]$. We briefly recall one. Let $L$ be a closed subgroup of $G$. Then a fundamental system of neighborhoods $V_{L}(K, V)$, where $K$ is a compact subset in $G$ and $V$ a neighborhood of the identity in $G$, of $L$ is defined as follows: a closed subgroup $M$ of $G$ belongs to $V_{L}(K, V)$ if $M \cap K \subset V \cdot L$ and $L \cap K \subset V \cdot M$.

Let $\Gamma \subset G$ be a discrete subgroup. Define

$$
i_{\Gamma}: \Gamma \backslash G \rightarrow \mathcal{S}(\mathbf{G}), \quad \Gamma g \rightarrow \Gamma^{g}=g^{-1} \Gamma g .
$$

If $\Gamma=\mathcal{N}(\Gamma)$, it is clearly injective. If moreover $\Gamma$ is arithmetic, then we shall show that $i_{\Gamma}$ is a homeomorphism of $\Gamma \backslash G$ onto its image. The proof uses the reduction theory, and the main point is the following proposition. 
Proposition 5.3. Assume that $\Gamma$ is arithmetic. Let $g_{j}$ be a divergent sequence in $\Gamma \backslash G$. If the sequence $\Gamma^{g_{j}}$ converges in $\mathcal{S}(G)$ to a closed subgroup $\Gamma_{\infty}$, then $\Gamma_{\infty}$ is not discrete.

We can of course replace $g_{j}$ by any element of $\Gamma g_{j}$. Taking this into account and using Proposition 2.5, we see that, after passing to a subsequence, we may assume that for some parabolic $\mathbb{Q}$-group $\mathbf{P}$, the sequence $g_{j}$ converges to $n_{\infty} m_{\infty}$ in $\bar{G}^{B S}$ for some $n_{\infty} \in N_{P}$ and $m_{\infty} \in M_{P} K$ (see $\S 3$ ). Then Proposition 5.3 follows from the following more precise result.

Proposition 5.4. Assume that $g_{j}$ converges to $n_{\infty} m_{\infty}$ in $\bar{G}^{B S}$ for some $n_{\infty} \in N_{P}$ and $m_{\infty} \in M_{P} K$. Then $\Gamma^{g_{j}}$ converges in $\mathcal{S}(G)$ to the group $m_{\infty}^{-1} N_{P} \Gamma_{P} m_{\infty}$.

Corollary 5.5. Assume $\Gamma=\mathcal{N}(\Gamma)$ and $\Gamma$ is arithmetic. Then $i_{\Gamma}$ : $\Gamma \backslash G \rightarrow \mathcal{S}(G)$ is a homeomorphism of $\Gamma \backslash G$ onto the image $i_{\Gamma}(\Gamma \backslash G)$.

Proof. We have to prove that $\Gamma g_{j}$ converges to $\Gamma g$ if and only if $\Gamma^{g_{j}}$ converges to $\Gamma^{g}$.

Assume that $\Gamma g_{j} \rightarrow \Gamma g$. Since $G \rightarrow \Gamma \backslash G$ is a covering map, we can choose $g_{j}$ such that $g_{j} \rightarrow g$, whence $\Gamma^{g_{j}} \rightarrow \Gamma^{g}$.

On the other hand, suppose that $\Gamma^{g_{j}} \rightarrow \Gamma^{g}$. It follows from Proposition 5.4 that we can assume $g_{j}$ to be bounded. Passing to a subsequence, if necessary, we may assume that $g_{j} \rightarrow g^{\prime} \in G$. Then $\Gamma^{g_{j}} \rightarrow \Gamma^{g^{\prime}}$, therefore $\Gamma^{g}=\Gamma^{g^{\prime}}$, hence $g^{\prime} g^{-1} \in \mathcal{N}(\Gamma)=\Gamma$ and $\Gamma g_{j} \rightarrow \Gamma g$. q.e.d.

Definition 5.6. Assume that $\Gamma$ is arithmetic and equal to its own normalizer $\mathcal{N}(\Gamma)$. We denote by $\Gamma \backslash \bar{G}^{s b}$ the closure of $i_{\Gamma}(\Gamma \backslash G)$ in $\mathcal{S}(G)$.

Since $i_{\Gamma}$ is a homeomorphism, $\Gamma \backslash \bar{G}^{s b}$ is a compactification of $\Gamma \backslash G$, and a $G$-space, to be called the subgroup compactification of $\Gamma \backslash G$.

Remark 5.7. For a non-maximal arithmetic subgroup $\Gamma$, the map $i_{\Gamma}: \Gamma \backslash G \rightarrow \mathcal{S}(G)$ is equal to the composition of

$$
\Gamma \backslash G \rightarrow \mathcal{N}(\Gamma) \backslash G, \quad \Gamma g \mapsto \mathcal{N}(\Gamma) g
$$

and the identification of $\mathcal{N}(\Gamma) \backslash G$ with the $G$-orbit of $\Gamma$ in $\mathcal{S}(G)$, and hence is a finite covering map to its image.

On the other hand, let $\Gamma_{\max }$ be a maximal discrete subgroup containing $\Gamma$. Then $\Gamma$ is of finite index in $\Gamma_{\max }$, and the composition of the covering $\Gamma \backslash G \rightarrow \Gamma_{\max } \backslash G$ and of the embedding $\Gamma_{\max } \backslash G \hookrightarrow \mathcal{S}(G)$ gives a map $\Gamma \backslash G \rightarrow \mathcal{S}(G)$ which is also a finite covering map onto its image of degree $\left[\Gamma_{\max }: \Gamma\right]$.

To study the compactification $\Gamma \backslash \bar{G}^{s b}$ intrinsically and to prove Proposition 5.4 above, we need to discuss the convergence in $\Gamma \backslash \bar{G}^{s b}$ of unbounded sequences in $\Gamma \backslash G$. 
Proposition 5.8. Let $g_{j}$ be a sequence satisfying the conditions in Proposition 5.4. Then $g_{j}^{-1} \Gamma_{P} g_{j} \rightarrow m_{\infty}^{-1} N_{P} \Gamma_{M_{P, x_{0}}} m_{\infty}$ in $\mathcal{S}(G)$.

Proof. The subscript $x_{0}$ will be dropped. By Proposition 2.8, $\Gamma_{P} \subseteq$ $N_{P} \Gamma_{M_{P}}$. This implies that $g_{j}^{-1} \Gamma_{P} g_{j} \subset g_{j}^{-1} N_{P} \Gamma_{M_{P}} g_{j}$. Since $g_{j}=$ $n_{j} a_{j} m_{j}$, we have

$$
\begin{aligned}
g_{j}^{-1} N_{P} \Gamma_{M_{P}} g_{j} & =m_{j}^{-1} a_{j}^{-1} N_{P} \Gamma_{M_{P}} n_{j} a_{j} m_{j} \\
& =m_{j}^{-1} N_{P} \Gamma_{M_{P}}\left(a_{j}^{-1} n_{j} a_{j}\right) m_{j} .
\end{aligned}
$$

Since $n_{j}$ is bounded, $a_{j}^{-1} n_{j} a_{j} \rightarrow i d$. This implies that $g_{j}^{-1} N_{P} \Gamma_{M_{P}} g_{j}$ converges to $m_{\infty}^{-1} N_{P} \Gamma_{M_{P}} m_{\infty}$ and hence, if any subsequence of $g_{j}^{-1} \Gamma_{P} g_{j}$ converges in $\mathcal{S}(G)$, the limit is contained in $m_{\infty}^{-1} N_{P} \Gamma_{M_{P}} m_{\infty}$.

We next show that all elements of $m_{\infty}^{-1} N_{P} \Gamma_{M_{P}} m_{\infty}$ are limits of sequences of points in $g_{j}^{-1} \Gamma_{P} g_{j}$. Hence the limit of $g_{j}^{-1} \Gamma_{P} g_{j}$ exists and is equal to $m_{\infty}^{-1} N_{P} \Gamma_{M_{P}} m_{\infty}$. For any $\gamma \in \Gamma_{M_{P}}$, we claim that $m_{\infty}^{-1} N_{P} \gamma m_{\infty}$ is contained in $\lim _{j \rightarrow+\infty} g_{j}^{-1} \Gamma_{P} g_{j}$. Since $\gamma \in \Gamma_{M_{P}}$ is arbitrary, this claim implies that $\lim _{j \rightarrow+\infty} g_{j}^{-1} \Gamma_{P} g_{j} \supseteq m_{\infty}^{-1} N_{P} \Gamma_{M_{P}} m_{\infty}$ and completes the proof of the proposition.

To prove the claim, we note that by Proposition 2.8, for every $\gamma \in$ $\Gamma_{M_{P}}$, there exists an element $n \in N_{P}$ such that $n \gamma \in \Gamma_{P}$, where $n$ is not necessarily in $\Gamma_{N_{P}}$. This implies that $\Gamma_{N_{P}} n \gamma \subset \Gamma_{P}$. Using $g_{j}=n_{j} a_{j} m_{j}$ again, we have

$$
\begin{aligned}
g_{j}^{-1} \Gamma_{N_{P}} n \gamma g_{j} & =m_{j}^{-1} a_{j}^{-1} n_{j}^{-1} \Gamma_{N_{P}} n \gamma n_{j} a_{j} m_{j} \\
& =m_{j}^{-1}\left(a_{j}^{-1} n_{j}^{-1} a_{j}\right)\left(a_{j}^{-1} \Gamma_{N_{P}} a_{j}\right)\left(a_{j}^{-1} n a_{j}\right) \gamma\left(a_{j}^{-1} n_{j} a_{j}\right) m_{j} .
\end{aligned}
$$

Since $n_{j}$ is bounded, $a_{j}^{-1} n_{j} a_{j}$ and $a_{j}^{-1} n_{j}^{-1} a_{j} \rightarrow i d$. Similarly, $a_{j}^{-1} n a_{j} \rightarrow$ $i d$. Since $\Gamma_{N_{P}}$ is a cocompact lattice in $N_{P}$, the exists a relatively compact open neighborhood $G$ of 1 in $N_{P}$ such that $N_{P}=C \cdot \Gamma_{N_{P}}$, whence also

$$
\left(a_{j}^{-1} \cdot C \cdot a_{j}\right) \cdot\left(a_{j}^{-1} \cdot \Gamma_{N_{P}} \cdot a_{j}\right)=N_{P} .
$$

But the $a_{j}^{-1} \cdot C \cdot a_{j}$ form a fundamental set of neighborhoods of 1 , hence any $n \in N_{P}$ is a limit of a sequence $a_{j}^{-1} \cdot \gamma_{j} \cdot a_{j}\left(\gamma_{j} \in \Gamma_{N_{P}}\right)$. q.e.d.

Proof of Proposition 5.4.

Write $\Gamma=\bigcup_{\gamma \in \Gamma / \Gamma_{P}} \gamma \Gamma_{P}$, where $\gamma$ runs over a set of representatives of $\Gamma / \Gamma_{P}$. Then

$$
g_{j}^{-1} \Gamma g_{j}=\bigcup_{\gamma \in \Gamma / \Gamma_{P}} g_{j}^{-1} \gamma \Gamma_{P} g_{j}=g_{j}^{-1} \Gamma_{P} g_{j} \cup \underset{\gamma \in \Gamma / \Gamma_{P}, \gamma \notin \Gamma_{P}}{\bigcup} g_{j}^{-1} \gamma \Gamma_{P} g_{j} .
$$


In view of Proposition 5.8, it suffices to prove that the sequence of subsets

$$
\bigcup_{\gamma \in \Gamma / \Gamma_{P}, \gamma \notin \Gamma_{P}} g_{j}^{-1} \gamma \Gamma_{P} g_{j}
$$

in $G$ goes to infinity. By assumption, $n_{j} \rightarrow n_{\infty}$ and $m_{j} \rightarrow m_{\infty}$. For simplicity, we assume that $n_{j}=i d, m_{j}=i d$, and hence $g_{j}=a_{j}$.

Note that it follows from Equation (2.1) that $P$ has a decomposition

$$
P=P^{-1}=M_{P} A_{P} N_{P} \cong M_{P} \times A_{P} \times N_{P} .
$$

This induces a decomposition of $G$ :

$$
G=K P=K M_{P} A_{P} N_{P} \cong K M_{P} \times A_{P} \times N_{P} .
$$

For any $g \in G$, write $g=m_{P}(g) a_{P}(g) n_{P}(g)=\left(m_{P}(g), a_{P}(g), n_{P}(g)\right) \in$ $K M_{P} \times A_{P} \times N_{P}$, and call $a_{P}(g)$ the $A_{P}$-component of $g$. (Note that the order of factors in this decomposition of $G$ is different from that in Equation 2.3 above.) The idea is to show that the $A_{P}$-component of the elements of the set in Equation (5.3) uniformly goes to infinity. We will use the fundamental representations of $\mathbf{G}$ defined over $\mathbb{Q}$ [Bo1, §14], $[\mathbf{B T}, \S 12]$ to prove this.

Let $\mathbf{P}_{0}$ be a minimal rational parabolic subgroup of $\mathbf{G}$ contained in P. For any simple $\mathbb{Q}$-root $\alpha \in \Delta\left(A_{P_{0}}, P_{0}\right)$, there is a strongly rational representation $\left(\pi_{\alpha}, \mathbf{V}_{\alpha}\right)$ of $\mathbf{G}$ whose highest weight $\lambda_{\alpha}$ is orthogonal to $\Delta\left(A_{P_{0}}, P_{0}\right)-\{\alpha\}$, and $\left\langle\lambda_{\alpha}, \alpha\right\rangle>0$. Then the weight space of $\lambda_{\alpha}$ is invariant under the maximal parabolic subgroup $P_{0, \Delta-\{\alpha\}}[\mathbf{B T}, \S 12.2$ and $\S 12.3]$. Fix an inner product \|\| on $\mathbf{V}_{\alpha}(\mathbb{R})$ which is invariant under $K$, and with respect to which $A_{P_{0}}$ is represented by self-adjoint operators. Let $e_{0}$ be a unit vector in the weight space of $\lambda_{\alpha}$. Let $P_{0, \Delta-\{\alpha\}}=M_{0, \Delta-\{\alpha\}} A_{0, \Delta-\{\alpha\}} N_{0, \Delta-\{\alpha\}}$ be the Langlands decomposition of $P_{0, \Delta-\{\alpha\}}$. Then for any $p \in M_{0, \Delta-\{\alpha\}} N_{0, \Delta-\{\alpha\}}$,

$$
\pi_{\alpha}(p) e_{0}= \pm e_{0}
$$

The Langlands decomposition $P_{0, \Delta-\{\alpha\}}=M_{P_{0, \Delta-\{\alpha\}}} A_{P_{0, \Delta-\{\alpha\}}} N_{P_{0, \Delta-\{\alpha\}}}$ induces the decomposition of $G$ :

$$
\begin{aligned}
G & =K M_{P_{0, \Delta-\{\alpha\}}} A_{P_{0, \Delta-\{\alpha\}}} N_{P_{0, \Delta-\{\alpha\}}} \\
& \cong K M_{P_{0, \Delta-\{\alpha\}}} \times A_{P_{0, \Delta-\{\alpha\}}} \times N_{P_{0, \Delta-\{\alpha\}}} .
\end{aligned}
$$

For any $g \in G$, denote the $A_{P_{0, \Delta-\{\alpha\}}}$-component by $a_{\Delta-\{\alpha\}}(g)$. Then

$$
\left\|\pi_{\alpha}(g) e_{0}\right\|=a_{\Delta-\{\alpha\}}(g)^{\lambda_{\alpha}}
$$

where $\lambda_{\alpha}$ is restricted to the subgroup $A_{P_{0, \Delta-\{\alpha\}}} \subseteq A_{P_{0}}$. If the $\mathbb{Q}$-parabolic subgroup $\mathbf{P}$ is contained in $\mathbf{P}_{0, \Delta-\{\alpha\}}$, then $M_{P} N_{P} \subseteq$ $M_{P_{0, \Delta-\{\alpha\}}} N_{P_{0, \Delta-\{\alpha\}}}$, and hence

$$
\left\|\pi_{\alpha}(g) e_{0}\right\|=a_{P}(g)^{\lambda_{\alpha}} .
$$


Now we follow the computations in [JM, pp. 505-506], [Bo7, pp. 550551]. For any $p \in \Gamma_{P}$, let

$$
g=a_{j}^{-1} \gamma p a_{j} .
$$

Since $\mathbf{P}_{0}$ is a minimal $\mathbb{Q}$-parabolic subgroup contained in $\mathbf{P}$, we can write $\mathbf{P}=\mathbf{P}_{0, I}$, where $I \subseteq \Delta=\Delta\left(P_{0}, A_{P_{0}}\right)$. For any $\alpha \in \Delta-I$, there are two cases: (1) $\gamma \in P_{0, \Delta-\{\alpha\}},(2) \gamma \notin P_{0, \Delta-\{\alpha\}}$.

In case (1), by Proposition 2.8, $\gamma p \in M_{P_{0, \Delta-\{\alpha\}}} N_{P_{0, \Delta-\{\alpha\}}}$, and hence $g=a_{j}^{-1} \gamma p a_{j} \in M_{P_{0, \Delta-\{\alpha\}}} N_{P_{0, \Delta-\{\alpha\}}}$. It follows that

$$
a_{P}(g)^{\lambda_{\alpha}}=1 .
$$

In case (2), using the Bruhat decomposition of $\mathbf{G}$ over $\mathbb{Q}$ [Bo1, $\S 11.4], \quad\left[\mathbf{J M}\right.$, Lemma 10.11], write $\gamma=u w t m v$, with $u \in N_{w}^{\prime}=$ $\left(w \mathbf{N}_{P_{0}^{-}} w^{-1}\right)(\mathbb{Q}) \cap \mathbf{N}_{P_{0}}(\mathbb{Q}), \mathbf{P}_{0}^{-}$being the minimal $\mathbb{Q}$-parabolic subgroup opposite to $\mathbf{P}_{0}, t \in A_{P_{0}}, m \in M_{P_{0}}, v \in N_{P_{0}}$, and $w$ is from a set of fixed representatives of the $\mathbb{Q}$-Weyl group of $\mathbf{G}$. Consider the element $w^{-1} g$ and its $A_{P^{-c o m p o n e n t}} a_{P}\left(w^{-1} g\right)$. Then the computations in $[\mathbf{J M}$, pp. 505-506] (also [Bo7, p. 551]) show that there exists a positive constant $\delta$ which only depends on $\Gamma$ and the fundamental representation $\pi_{\alpha}$ such that

$$
a_{P}\left(w^{-1} g\right)^{\lambda_{\alpha}} \geq a_{j}^{\alpha} \delta
$$

This implies that when $j \rightarrow+\infty, w^{-1} g$ and hence $g$ goes to infinity uniformly with respect to an arbitrary choice of $p \in \Gamma_{P}$.

Note that $P=P_{0, I}=\cap_{\alpha} P_{0, \Delta-\{\alpha\}}$, where $\alpha \in \Delta-I$. Hence for any $\gamma \notin \Gamma_{P}$, there exists at least one $\alpha \in \Delta-I$ such that $\gamma \notin P_{0, \Delta-\{\alpha\}}$. Since the set of $w$ is a fixed finite set, this implies that when $j \rightarrow+\infty$, the subset of $G$ defined in Equation (5.3) goes to infinity. This completes the proof of the proposition.

\section{Remarks 5.9.}

(1) The limit subgroup $N_{P} \Gamma_{M_{P}}$ in Proposition 5.4 is exactly the subgroup which leaves invariant the constant term of automorphic forms, in particular Eisenstein series, along the parabolic subgroup $P$. This interpretation of the boundary subgroups in $\Gamma \backslash \bar{G}^{s b}$ makes it an interesting compactification.

(2) The above proof of Proposition 5.4 is related to determination of asymptotic behaviors at infinity of Eisenstein series of $P$. Specifically, let $E(P \mid 1, \Lambda: x)$ be the Eisenstein series of $P$ associated with the constant function 1 on $\Gamma_{M_{P}} \backslash X_{P}$, where $\Lambda \in \mathfrak{a}_{P}^{*}$. (See [JM, §13.2] for more details about the definition of Eisenstein series.) When $\Lambda \gg 0$ with respect to the positive chamber determined by $P, E(P \mid 1, \Lambda: x)$ converges absolutely. It is known that the asymptotic behaviors (or sizes) of $E(P \mid 1, \Lambda: x)$ (or more generally an automorphic form) are controlled by the constant terms, which consist of finitely many terms 
(see [HC, Chap. I, $\S 4$, Chap. II, $\S 4, \S 5]$ for definitions and related discussions). The constant terms of an Eisenstein series associated with a cusp form can be computed rather explicitly (see [HC, Theorem 5, p. 44, and Corollary 2, p. 39]). On the other hand, the constant terms of an Eisenstein series associated with a non-cuspidal function, for example $E(P \mid 1, \Lambda: x)$ associated with the constant function 1, are not so well-understood. The above proof seems to indicate that when $x=e^{H_{j}} \cdot x_{0}$ and $\alpha\left(H_{j}\right) \rightarrow+\infty$ for all $\alpha \in \Delta\left(A_{P}, P\right)$, the leading term of the constant terms of $E\left(P \mid 1, \Lambda: e^{H_{j}} \cdot x_{0}\right)$ is equal to $e^{\left(\rho_{P}+\Lambda\right)\left(H_{j}\right)}$.

Proposition 5.10. Assume that $\Gamma$ is a maximal arithmetic subgroup. Then the identity map on $\Gamma \backslash G$ extends to a continuous map from $\Gamma \backslash \bar{G}^{R B S}$ to $\Gamma \backslash \bar{G}^{\text {sb }}$ which is surjective and equivariant with respect to the right $G$-action.

Proof. Let $\mathbf{P}$ be a parabolic $\mathbb{Q}$-subgroup. Let $g_{j}$ be an unbounded sequence in $\Gamma \backslash G$ converging to $m_{\infty} \in \Gamma_{M_{P}} \backslash \hat{e}(P)$ in $\Gamma \backslash \bar{G}^{R B S}$. Since $\Gamma_{N_{P}}$ is a cocompact lattice in $N_{P}$, we can choose a lift $\tilde{g}_{j}$ in $G$ such that in the decomposition $\tilde{g}_{j}=\left(n_{j}, a_{j}, m_{j}\right) \in N_{P} \times A_{P} \times\left(M_{P} K\right)$, the component $n_{j}$ is bounded, and the component $m_{j}$ converges to a lift $\tilde{m}_{\infty}$ of $m_{\infty}$ in $M_{P} K=\hat{e}(P)$.

By the definition of the convergence in $\Gamma \backslash \bar{G}^{R B S}$, we know that for all $\alpha \in \Phi\left(P, A_{P}\right),\left(a_{j}\right)^{\alpha} \rightarrow+\infty$. Then by Proposition 5.8, $\tilde{g}_{j}$ converges in $\Gamma \backslash \bar{G}^{s b}$ to $\tilde{m}_{\infty}^{-1} N_{P} \Gamma_{M_{P}} \tilde{m}_{\infty}$, i.e., the subgroup $\tilde{g}_{j}^{-1} \Gamma \tilde{g}_{j}$ converges to $\tilde{m}_{\infty}^{-1} N_{P} \Gamma_{M_{P}} \tilde{m}_{\infty}$. Since $g_{j}^{-1} \Gamma g_{j}=\tilde{g}_{j}^{-1} \Gamma \tilde{g}_{j}$ and the limit $\tilde{m}_{\infty}^{-1} N_{P} \Gamma_{M_{P}} \tilde{m}_{\infty}$ $=m_{\infty}^{-1} N_{P} \Gamma_{M_{P}} m_{\infty}$ does not depend on the choice of the lift $\tilde{m}_{\infty}$ in $G$, $g_{j}$ converges in $\Gamma \backslash \bar{G}^{s b}$. This shows that every unbounded sequence in $\Gamma \backslash G$ which is convergent in $\Gamma \backslash \bar{G}^{R B S}$ also converges in $\Gamma \backslash \bar{G}^{s b}$. Since both $\Gamma \backslash \bar{G}^{R B S}$ and $\Gamma \backslash \bar{G}^{s b}$ are metrizable compactifications of $\Gamma \backslash G$, by [GJT, Lemma 3.28], the identity map on $\Gamma \backslash G$ extends to a continuous map from $\Gamma \backslash \bar{G}^{R B S}$ to $\Gamma \backslash \bar{G}^{s b}$, which is automatically surjective, and the extended map is $G$-equivariant with respect to the right $G$-action. q.e.d.

Definition 5.11. Two parabolic $\mathbb{Q}$-subgroups $\mathbf{P}_{1}, \mathbf{P}_{2}$ are called $\Gamma_{M^{-}}$ equivalent if there exists $g \in G$ such that $g^{-1} N_{P_{1}} \Gamma_{M_{P_{1}}} g=N_{P_{2}} \Gamma_{M_{P_{2}}}$.

Since $N_{P_{i}}$ is the identity component of $N_{P_{i}} \Gamma_{M_{P_{i}}}$, the normalizer of $N_{P_{i}} \Gamma_{M_{P_{i}}}$ is contained in the normalizer of $N_{P_{i}}$, hence in $P_{i}$ (see Remark 2.10). This implies that if $\mathbf{P}_{1}, \mathbf{P}_{2}$ are $\Gamma_{M}$-equivalent, $\mathbf{P}_{1}, \mathbf{P}_{2}$ are conjugate under $G$ and hence also under $\mathbf{G}(\mathbb{Q})$. On the other hand, if $\mathbf{P}_{1}, \mathbf{P}_{2}$ are $\Gamma$-conjugate, they are clearly $\Gamma_{M}$-equivalent. Let $\mathbf{P}_{1}, \ldots, \mathbf{P}_{k}$ be a set of representatives of the $\Gamma$-conjugacy classes of parabolic $\mathbb{Q}$-subgroups. Then there exists a subset of $\mathbf{P}_{1}, \ldots, \mathbf{P}_{k}$ which are representatives of the $\Gamma_{M}$-equivalent classes of parabolic $\mathbb{Q}$-subgroups. For simplicity, assume that they are given by $\mathbf{P}_{1}, \ldots, \mathbf{P}_{l}$ for some $l \leq k$. 
Lemma 5.12. For every $i$, the normalizer $\mathcal{N}\left(N_{P_{i}} \Gamma_{M_{P_{i}}}\right)$ of $N_{P_{i}} \Gamma_{M_{P_{i}}}$ in $M_{P_{i}} K$ is equal to the normalizer $\mathcal{N}\left(\Gamma_{M_{P_{i}}}\right)$ of $\Gamma_{M_{P_{i}}}$ in $M_{P_{i}}$.

Proof. If an element normalizes $N_{P_{i}} \Gamma_{M_{P_{i}}}$, then it normalizes its identity component $N_{P_{i}}$. It follows from [BT, Proposition 3.1] that the normalizer of $N_{P_{i}}$ in $G$ is equal to $P_{i}$. In fact, Proposition 3.1 in [BT] shows that there is a rational parabolic subgroup $\mathbf{P}$ which contains the normalizer of $N_{P_{i}}$ and whose unipotent radical is equal to $N_{P_{i}}$. Clearly, such a parabolic subgroup has to be exactly equal to $\mathbf{P}_{i}$. Then it is clear that the normalizer of $N_{P_{i}} \Gamma_{M_{P_{i}}}$ in $M_{P_{i}} K$ is contained in $M_{P_{i}}$ and hence is equal to the normalizer $\mathcal{N}\left(\Gamma_{M_{P_{i}}}\right)$ of $\Gamma_{M_{P_{i}}}$ in $M_{P_{i}}$. $\quad$ q.e.d.

Theorem 5.13. Keep the notation of 5.11 and 5.12. Assume $\Gamma$ to be a maximal arithmetic subgroup. Then

$$
\Gamma \backslash \bar{G}^{s b}=\Gamma \backslash G \cup \coprod_{i=1}^{l} \mathcal{N}\left(\Gamma_{M_{P_{i}}}\right) \backslash M_{P_{i}} K
$$

is the decomposition of $\Gamma \backslash \bar{G}^{s b}$ into G-orbits.

Proof. It follows from Propositions 5.8 and 5.10 that under the map

$$
\Gamma \backslash \bar{G}^{R B S} \rightarrow \Gamma \backslash \bar{G}^{s b},
$$

the image, denoted by $b\left(P_{i}\right)$, of the boundary component $\Gamma_{M_{P_{i}}} \backslash \hat{e}\left(P_{i}\right)$ of $\Gamma \backslash \bar{G}^{R B S}$ consists of subgroups of the form $m^{-1} N_{P_{i}} \Gamma_{M_{P_{i}}} m$, where $m \in M_{P_{i}} K, i=1, \ldots, k$. By Lemma 5.12 , the normalizer of $N_{P_{i}} \Gamma_{M_{P_{i}}}$ in $M_{P_{i}} K$ is equal to the normalizer $\mathcal{N}\left(\Gamma_{M_{P_{i}}}\right)$ of $\Gamma_{M_{P_{i}}}$ in $M_{P_{i}}$, and hence we obtain that the image $b\left(P_{i}\right)$ can be identified with $\mathcal{N}\left(\Gamma_{M_{P_{i}}}\right) \backslash M_{P_{i}} K$ through the map $m \rightarrow m^{-1} N_{P_{i}} \Gamma_{M_{P_{i}}} m$.

Since each boundary component $\Gamma_{M_{P_{i}}} \backslash \hat{e}\left(P_{i}\right)$ of $\Gamma \backslash \bar{G}^{R B S}$ is a $G$-orbit (Proposition 4.9) and the map $\Gamma \backslash \bar{G}^{R B S} \rightarrow \Gamma \backslash \bar{G}^{B S}$ is G-equivariant (Proposition 5.10), the image $b\left(P_{i}\right)$ is also a $G$-orbit. In fact, for $m^{-1} N_{P_{i}} \Gamma_{M_{P_{i}}} m \in b\left(P_{i}\right)$, and $g \in G$,

$$
g \circ m^{-1} N_{P_{i}} \Gamma_{M_{P_{i}}} m=g^{-1} m^{-1} N_{P_{i}} \Gamma_{M_{P_{i}}} m g .
$$

From this, it is clear that two image sets $b\left(P_{i}\right), b\left(P_{j}\right)$ are equal if and only if $P_{i}$ and $P_{j}$ are $\Gamma_{M}$-equivalent. This gives the disjoint decomposition of $\Gamma \backslash \bar{G}^{s b}$ in the theorem, and shows that the decomposition is exactly the decomposition into the disjoint $G$-orbits on $\Gamma \backslash \bar{G}^{s b}$. $\quad$ q.e.d.

Proposition 5.14. For any arithmetic subgroup $\Gamma$, let $\Gamma^{\prime}$ be a maximal discrete group containing $\Gamma$. Then the projection map $\Gamma \backslash G \rightarrow \Gamma^{\prime} \backslash G$ extends to a continuous map $\Gamma \backslash \bar{G}^{R B S} \rightarrow{\overline{\Gamma^{\prime} \backslash G}}^{\text {sb }}$. 
Proof. We note that the quotient map $\Gamma \backslash G \rightarrow \Gamma^{\prime} \backslash G$ extends to a continuous map $\Gamma \backslash \bar{G}^{R B S} \rightarrow \Gamma^{\prime} \backslash \bar{G}^{R B S}$. Then the proposition follows from Proposition 5.10.

q.e.d.

Proposition 5.15. Suppose that $\mathbf{G}$ is a semisimple algebraic group defined over $\mathbb{Q}, \Gamma \subset \mathbf{G}(\mathbb{Q})$ is a maximal arithmetic subgroup, and the $\Gamma$-conjugacy relation on the set of all parabolic $\mathbb{Q}$-subgroups induces the same relation as the $\Gamma_{M}$-equivalence relation in Definition 5.11. If for every parabolic $\mathbb{Q}$-subgroup $\mathbf{P}, M_{P}$ is semisimple without compact factor of positive dimension, and its subgroup $\Gamma_{M_{P}}$ is also maximal, then $\Gamma \backslash \bar{G}^{s b}$ is G-equivariantly isomorphic to the reductive Borel-Serre compactification $\Gamma \backslash \bar{G}^{R B S}$.

Proof. Let $\mathbf{P}_{1}, \ldots, \mathbf{P}_{k}$ be a set of representatives of $\Gamma$-conjugacy classes of proper parabolic $\mathbb{Q}$-subgroups. By assumption, they are also representatives of the $\Gamma_{M}$-relation. Since $\mathcal{N}\left(\Gamma_{M_{P_{i}}}\right)=\Gamma_{M_{P_{i}}}$, by Theorem 5.13 , the boundary of $\Gamma \backslash \bar{G}^{s b}$ is equal to

$$
\bigcup_{i=1}^{k} \Gamma_{M_{P_{i}}} \backslash M_{P_{i}} K=\bigcup_{i=1}^{k} \Gamma_{M_{P_{i}}} \backslash \hat{e}\left(P_{i}\right),
$$

which is also the boundary of $\Gamma \backslash \bar{G}^{R B S}$. This implies that the continuous map from $\Gamma \backslash \bar{G}^{R B S}$ to $\Gamma \backslash \bar{G}^{s b}$ in Proposition 5.10 is bijective. Since both compactifications are Hausdorff, they are homeomorphic, and the homeomorphism is equivariant with respect to the right $G$-action. q.e.d.

Remark 5.16. Examples where all the conditions in the above theorem are satisfied include $G=S L(n, \mathbb{R}), S p(n, \mathbb{R}), \Gamma=S L(n, \mathbb{Z})$, $S p(n, \mathbb{Z})$.

\section{Remarks 5.17.}

(1) If $\Gamma$ is maximal, but other conditions are not satisfied, then $M_{P_{i}}$ is in general only reductive. Let $M_{P_{i}}^{\prime}$ be the derived group of $M_{P_{i}}$, and $\mathcal{C}\left(M_{P_{i}}\right)$ the center of $M_{P_{i}}$. Then $\mathcal{N}\left(\Gamma_{M_{P_{i}}}\right)$ contains $\mathcal{C}\left(M_{P_{i}}\right)$, and $\mathcal{C}\left(M_{P_{i}}\right) \backslash \mathcal{N}\left(\Gamma_{M_{P_{i}}}\right)$ is a discrete subgroup of $M_{P_{i}}^{\prime}$, and $\mathcal{N}\left(\Gamma_{M_{P_{i}}}\right) \backslash M_{P_{i}} K$ is equal to $\left(\mathcal{C}\left(M_{P_{i}}\right)\right) \backslash \mathcal{N}\left(\Gamma_{M_{P_{i}}}\right) \backslash M_{P_{i}}^{\prime} K$. This shows that the boundary components of $\Gamma \backslash \bar{G}^{s b}$ are analogous to the boundary components in the maximal Satake compactification of $\Gamma \backslash X$ in [Sa2].

(2) To obtain a compactification of $\Gamma \backslash X$ using this method, consider the compact space $\mathcal{S}(G) / K$ of $K$-orbits in $\mathcal{S}(G)$, and the map $\Gamma \backslash X \rightarrow$ $\mathcal{S}(G) / K, \quad \Gamma g K \mapsto K \Gamma^{g} K$. 


\section{Analytic structures on $\Gamma \backslash \bar{G}^{B S}$ and $\Gamma \backslash \bar{X}^{B S}$}

In this section we show that $\bar{G}^{B S}$ is a real analytic manifold with corners and hence $\Gamma \backslash \bar{G}^{B S}$ is also a real analytic manifold with corners when $\Gamma$ is neat. The same proof also shows the Borel-Serre partial compactification $\bar{X}^{B S}$ and the Borel-Serre compactification $\Gamma \backslash \bar{X}^{B S}$ when $\Gamma$ is neat are also real analytic manifolds with corners, which were obtained in $[\mathbf{B S}]$.

Recall from $\S 2.4$ that for any parabolic $\mathbb{Q}$-subgroup $\mathbf{P}, \Delta\left(P, A_{P}\right)=$ $\left\{\alpha_{1}, \ldots, \alpha_{r}\right\}$ is the set of simple roots in $\Phi\left(P, A_{P}\right)$. Then $A_{P}$ can be identified with $\left(\mathbb{R}_{>0}\right)^{r}$ by the map:

$$
a \in A_{P} \rightarrow\left(a^{-\alpha_{1}}, \ldots, a^{-\alpha_{r}}\right) \in\left(\mathbb{R}_{>0}\right)^{r} .
$$

The closure of $A_{P}$ in $\mathbb{R}^{r}$ is denoted by $\overline{A_{P}}$, a partial compactification in the direction of $P$.

By Proposition 3.3, the embedding $N_{P} \times A_{P} \times M_{P} K \hookrightarrow \bar{G}^{B S}$ extends to an embedding

$$
N_{P} \times \overline{A_{P}} \times M_{P} K=G \hookrightarrow \bar{G}^{B S},
$$

and the image of $N_{P} \times \overline{A_{P}} \times M_{P} K$ in $\bar{G}^{B S}$ is the corner $G(P)$ associated with $\mathbf{P}$.

Proposition 6.1. For every parabolic $\mathbb{Q}$-subgroup $\mathbf{P}$, the corner $G(P)$ has a canonical structure of real analytic manifold with corners compatible with the interior analytic structure of $G$.

Proof. By definition,

$$
G(P) \cong N_{P} \times\left(\mathbb{R}_{\geq 0}\right)^{r} \times\left(M_{P} K\right) .
$$

Since $N_{P}, M_{P}$ are real analytic manifolds, and $\left(\mathbb{R}_{\geq 0}\right)^{r}$ is a real analytic manifold with corners, $N_{P} \times\left(\mathbb{R}_{\geq 0}\right)^{r} \times\left(M_{P} K\right)$ is a real analytic manifold with corners, which gives $G(P)$ a structure of real analytic manifold with corners. Since the horospherical decomposition $G=N_{P} \times A_{P} \times M_{P} K$ is real analytic, this real analytic structure on $G(P)$ is compatible with the real analytic structure of $G$. $\quad$ q.e.d.

These corners $G(P)$ for all $\mathbf{P}$ form a covering family of $\bar{G}^{B S}$. To show that they define a real analytic structure on $\bar{G}^{B S}$, we need to prove that these structures are compatible.

By Proposition 3.3, the corner $G(P)$ admits a disjoint decomposition:

$$
G(P)=G \cup \coprod_{Q \supseteq P} e(Q) .
$$

This implies that for any two parabolic $\mathbb{Q}$-subgroups $\mathbf{P}, \mathbf{Q}$ with $\mathbf{P} \subset \mathbf{Q}$, $G(Q)$ is canonically contained in $G(P)$. 
Proposition 6.2. For any two parabolic $\mathbb{Q}$-subgroups $\mathbf{P} \subset \mathbf{Q}$, the inclusion $G(Q) \hookrightarrow G(P)$ is real analytic with respect to the canonical real analytic structure on $G(P), G(Q)$ defined in Proposition 6.1, and embeds $G(Q)$ as an open real analytic submanifold with corners of $G(P)$.

Proof. As in $\S 3, P$ determines a unique parabolic subgroup $P^{\prime}$ of $M_{Q}$, $P^{\prime}=M_{Q} \cap P$, such that

$$
A_{P}=A_{P^{\prime}} A_{Q}, N_{P}=N_{P^{\prime}} N_{Q}, M_{P^{\prime}}=M_{P} .
$$

This $P^{\prime}$ induces a decomposition of $M_{Q}: M_{Q}=N_{P^{\prime}} \times A_{P^{\prime}} \times M_{P^{\prime}}$ and hence a refined horospherical decomposition of $G$ with respect to $Q$ :

$$
G=N_{P} \times A_{P^{\prime}} \times A_{Q} \times\left(M_{P} K\right) .
$$

Since these decompositions are real analytic, this implies that the corner $G(Q)$ is real analytically diffeomorphic to

$$
N_{P} \times A_{P^{\prime}} \times \overline{A_{Q}} \times\left(M_{P} K\right) \cong N_{P} \times A_{P^{\prime}} \times\left(\mathbb{R}_{\geq 0}\right)^{r_{Q}} \times\left(M_{P} K\right),
$$

where $r_{Q}=\operatorname{dim} A_{Q}$ is the $\operatorname{rank}$ of $Q$.

Let $I$ be the subset of the set $\Delta\left(P, A_{P}\right)$ of simple roots such that $Q=P_{I}$, i.e., $A_{Q}=\left\{a \in A_{P} \mid a^{\alpha}=1, \alpha \in I\right\}$. For simplicity, assume that $I=\left\{\alpha_{r_{Q}+1}, \ldots, \alpha_{r}\right\}$. Then $A_{P, Q}=\left\{a \in A_{P} \mid \alpha^{\alpha_{i}}=1,1 \leq i \leq r_{Q}\right\}$ can be identified with $\left(\mathbb{R}_{>0}\right)^{r-r_{Q}}$ through the map

$$
a \in A_{P, Q} \rightarrow\left(a^{-\alpha_{r_{Q}+1}}, \ldots, a^{-\alpha_{r}}\right) \in\left(\mathbb{R}_{>0}\right)^{r-r_{Q}} .
$$

Define $\overline{A_{P, Q}}$ to be the closure of $A_{P, Q}$ in $\mathbb{R}^{r-r_{Q}}$. Then the product $\mathbb{R}^{r}=\mathbb{R}^{r-r_{Q}} \times \mathbb{R}^{r_{Q}}$ gives a decomposition $\overline{A_{P}}=\overline{A_{P, Q}} \times \overline{A_{Q}}$. This implies that

$$
G(P)=N_{P} \times \overline{A_{P, Q}} \times \overline{A_{Q}} \times M_{P} K .
$$

By Lemmas 3.5 and 3.6, $G(Q)$ is identified under the inclusion $G(Q) \subset$ $G(P)$ with the subset $N_{P} \times A_{P, Q} \times \overline{A_{Q}} \times M_{P} K \subset G(P)$. Clearly, $N_{P} \times A_{P, Q} \times \overline{A_{Q}} \times M_{P} K$ is an open real analytic submanifold of $G(P)$. By the previous paragraph, $G(Q)=N_{P} \times A_{P^{\prime}} \times \overline{A_{Q}} \times\left(M_{P} K\right)$. Then the proof of the proposition is reduced to showing that the coordinate change function

$$
N_{P} \times A_{P^{\prime}} \times A_{Q} \times M_{P} K \rightarrow N_{P} \times A_{P, Q} \times A_{Q} \times M_{P} K
$$

extends to a real analytic diffeomorphism

$$
N_{P} \times A_{P^{\prime}} \times \overline{A_{Q}} \times M_{P} K \rightarrow N_{P} \times A_{P, Q} \times \overline{A_{Q}} \times M_{P} K .
$$

By the proof of Lemma 3.6, the coordinate change function

$$
N_{P} \times A_{P^{\prime}} \times A_{Q} \times M_{P} K \rightarrow N_{P} \times A_{P, Q} \times A_{Q} \times M_{P} K
$$

is given by

$$
(n, \exp H, \exp V, m) \mapsto\left(n, \exp H_{P, Q}, \exp \left(V+H_{Q}\right), m\right),
$$


where $H=H_{P, Q}+H_{Q}, H_{P, Q} \in \mathfrak{a}_{P, Q}, H_{Q} \in \mathfrak{a}_{Q}$. We claim that both $H_{P, Q}$ and $H_{Q}$ depend real analytically on $H$ and the map

$$
H \in \mathfrak{a}_{P^{\prime}} \rightarrow H_{P, Q} \in \mathfrak{a}_{P, Q}
$$

is a real analytic diffeomorphism. In fact, let $V_{1}, \ldots, V_{r}$ be the basis of $\mathfrak{a}_{P}$ dual to the simple roots $\alpha_{1}, \ldots, \alpha_{r}$. Then $H_{P, Q}=\alpha_{r_{Q}+1}(H) V_{r_{Q}+1}+$ $\cdots+\alpha_{r}(H) V_{r}, H_{Q}=\alpha_{1}(H) V_{1}+\cdots+\alpha_{r_{Q}}(H) V_{r_{Q}}$. This clearly gives analytic dependence of $H_{P, Q}, H_{Q}$ on $H$. Since $\mathfrak{a}=\mathfrak{a}_{P^{\prime}} \oplus \mathfrak{a}_{Q}=\mathfrak{a}_{P, Q} \oplus \mathfrak{a}_{Q}$, the map $H \in \mathfrak{a}_{P^{\prime}} \rightarrow H_{P, Q} \in \mathfrak{a}_{P, Q}$ is a linear isomorphism and hence a real analytic diffeomorphism.

With respect to the canonical analytic structure of $G(Q), G(Q)=$ $N_{P} \times A_{P^{\prime}} \times\left(\mathbb{R}_{\geq 0}\right)^{r_{Q}} \times M_{P} K$, and the coordinates of $g=(n, \exp H$, $\exp V, m) \in N_{P} \times A_{P^{\prime}} \times A_{Q} \times M_{P} K=G$ in this decomposition of $G(Q)$ are $\left(n, \exp H ; e^{-\alpha_{1}(V)}, \ldots, e^{-\alpha_{r}(V)} ; m\right)$. On the other hand, with respect to the canonical analytic structure of $G(P)$,

$$
G(P)=N_{P} \times \overline{A_{P, Q}} \times\left(\mathbb{R}_{\geq 0}\right)^{r_{Q}} \times M_{P} K,
$$

and the coordinates of the same element $g$ in this decomposition of $G(P)$ are

$$
\left(n, \exp H_{P, Q} ; e^{-\alpha_{1}(V)} e^{-\alpha_{1}\left(H_{Q}\right)}, \ldots, e^{-\alpha_{r}(V)} e^{-\alpha_{r}\left(H_{Q}\right)} ; m\right) .
$$

This implies that the coordinate change function from $N_{P} \times A_{P^{\prime}} \times A_{Q} \times$ $M_{P} K=N_{P} \times A_{P^{\prime}} \times\left(\mathbb{R}_{>0}\right)^{r} \times M_{P} K$ to $N_{P} \times A_{P, Q} \times A_{Q} \times M_{P} K=$ $N_{P} \times A_{P, Q} \times\left(\mathbb{R}_{>0}\right)^{r} \times M_{P} K$ extends to $N_{P} \times A_{P^{\prime}} \times\left(\mathbb{R}_{\geq 0}\right)^{r} \times M_{P} K \rightarrow$ $N_{P} \times A_{P, Q} \times\left(\mathbb{R}_{\geq 0}\right)^{r_{Q}} \times M_{P} K$, given by $\left(n, \exp H ; t_{1}, \ldots, t_{r_{Q}} ; m\right) \mapsto$ $\left(n, \exp H_{P, Q} ; t_{1} e^{-\alpha_{1}\left(H_{Q}\right)}, \ldots, t_{r} e^{-\alpha_{r}\left(H_{Q}\right)} ; m\right)$. Since $H_{P, Q}, H_{Q}$ depend real analytically on $H$ and the map $H \in \mathfrak{a}_{P^{\prime}} \rightarrow H_{P, Q} \in \mathfrak{a}_{P, Q}$ is a real analytic diffeomorphism, the above extended coordinate change function is a real analytic diffeomorphism also. This completes the proof of the proposition.

q.e.d.

Proposition 6.3. The partial compactification $\bar{G}^{B S}$ is a real analytic manifold with corners and the restriction to each corner $G(P)$ gives the canonical analytic structure in Proposition 6.1.

Proof. We first show that every corner $G(P)$ is an open subset of $\bar{G}^{B S}$. In fact, by Lemma 3.10 , for any $p \in e(P), G(P)$ contains a neighborhood of $p$ in $\bar{G}^{B S}$. For any $Q \supset P$ and a point $q \in G(Q), G(P)$ contains $G(Q)$ and hence also contains a neighborhood of $q$ in $\bar{G}^{B S}$.

Since $\bar{G}^{B S}=G \cup \coprod_{P} e(P)=\cup G(P)$, these corners form an open covering of $\bar{G}^{B S}$. By Proposition 6.1, each corner has a canonical structure of a real analytic manifold with corners, and hence these corners form a system of charts of $\bar{G}^{B S}$. To finish the proof, we only need to show that these structures of the corners are compatible. 
For any two parabolic $\mathbb{Q}$-subgroups $\mathbf{P}_{1}, \mathbf{P}_{2}$, let $\mathbf{Q}$ be the smallest, not necessarily proper, parabolic subgroup containing both $P_{1}, P_{2}$. Since $G\left(P_{i}\right)=G \cup \coprod_{R \supset P_{i}} e(R)$, we have that

$$
G\left(P_{1}\right) \cap G\left(P_{2}\right)=G(Q),
$$

where $G(Q)=G$ if $\mathbf{Q}=\mathbf{G}$. By Proposition 6.2, the canonical analytic structure of each $G\left(P_{i}\right)$ is compatible with the canonical structure of $G(Q)$. This implies that the canonical analytic structures of $G\left(P_{1}\right)$, $G\left(P_{2}\right)$ are compatible on their intersection, and hence completes the proof.

q.e.d.

Proposition 6.4. When $\Gamma$ is a neat arithmetic subgroup, $\Gamma \backslash \bar{G}^{B S}$ is a compact real analytic manifold with corners.

Proof. When $\Gamma$ is neat, for any parabolic $\mathbb{Q}$-subgroup $\mathbf{P}$, the induced subgroups $\Gamma_{P}, \Gamma_{N_{P}}, \Gamma_{M_{P}}$ are all torsion free. This implies that $\gamma$ acts freely and proper discontinuously on $\bar{G}^{B S}$. By Proposition $6.3, \bar{G}^{B S}$ is a real analytic manifold with corners. To finish the proof, it suffices to show that $\Gamma$ acts on $\bar{G}^{B S}$ by analytic diffeomorphism.

For any $\gamma \in \Gamma$, if $\gamma \mathbf{P} \gamma^{-1}=\mathbf{P}^{\prime}$, then by Proposition 3.3 and the proof of Proposition 3.12, the left action on $\bar{G}^{B S}$ maps the corner $G(P)$ to $G\left(P^{\prime}\right)$. It can also be seen from the proof that the induced map on the corners $\gamma: G(P) \rightarrow G\left(P^{\prime}\right)$ is real analytic. In fact, the Langlands decomposition is real analytic, and the $A_{P}$-component is changed only by the $A_{P}$-component of $\gamma$. This implies the desired analyticity. Since $\gamma^{-1}$ maps $G\left(P^{\prime}\right)$ to $G(P)$ real analytically, it follows that $\gamma$ acts by analytic diffeomorphism on $\bar{G}^{B S}$.

q.e.d.

\section{Gluing manifolds with corners}

This section gives an exposition of the self-gluing procedure in [BJ1, 4.4]. The notion of manifold with corners is assumed to be known (see the appendix in $[\mathbf{B S}]$ by Douady and Herault). We review only some facts and notation.

Let $M$ a connected manifold with corners, $m$ its dimension. Every point $p \in M$ has a local chart of the form $\mathbb{R}^{m-i} \times \mathbb{R}_{\geq 0}^{i}$, where $R_{\geq 0}^{i}$ is a positive (closed) quadrant in $\mathbb{R}^{i}$ and $p$ is mapped to the origin. The integer $i$ is called the rank of $p$, and the maximum of $i$ is called the rank of $M$, denoted by $r k(M)$. The manifold $M$ has a stratification such that each stratum consists of points of the same rank. Every connected component of a stratum is called an open boundary face of $M$, and its closure in $M$ is called a boundary face. If a boundary face is of codimension 1 , it is called a boundary hypersurface.

The boundary $\partial M$ of $M$ is the union of boundary hypersurfaces, which are themselves manifolds with corners of rank strictly less than 
$r k(M)$. We shall assume they are all of rank equal to $r k(M)-1$ and embedded (no self-intersection). More precisely, a boundary hypersurface $H$ is embedded if for every point $p$ of rank $i$ and belonging the boundary of $H$, there exist $i-1$ boundary hypersurfaces $H_{1}, \ldots, H_{i-1}$ different from $H$ such that $p$ belongs to the intersection $H \cap H_{1} \cdots \cap H_{i-1}$ and the intersection has codimension $i$, which is automatically satisfied if all $H_{1}, \ldots, H_{i-1}$ are different.

If all the boundary hypersurfaces are embedded, the intersection of two boundary hypersurfaces is a manifold with corners of rank equal to $r k(M)-2$ (if not empty), and is union of boundary hypersurfaces of each of them, considered as manifolds with corners. For any boundary face of $M$ of codimension $i$, its boundary hypersurfaces are also embedded if all the boundary hypersurfaces of $M$ are embedded, and they are intersections of $i+1$ boundary hypersurfaces of $M$.

An example of a manifold with corners whose boundary hypersurfaces are not embedded is a 2-dimensional manifold with one corner point and one boundary hypersurface. It is clear that this two dimensional manifold can not be self-glued into a closed smooth manifold. Therefore, the assumption that boundary hypersurfaces are embedded is crucial.

Our aim here is to glue $M$ to a certain number of copies of itself so as to get a smooth manifold, and to give an alternate formulation in the case where it is possible to use the smallest possible number of copies of $M$, namely $2^{r k(M)}$. For the gluing purpose, we need to assume that the set $\mathcal{H}_{M}$ of boundary hypersurfaces of $M$ admits a finite partition:

$$
\mathcal{H}_{M}=\coprod_{j=1}^{N} \mathcal{H}_{j}
$$

such that the elements of each $\mathcal{H}_{j}$ are disjoint $(1 \leq j \leq N)$. If $M$ is compact, then $\mathcal{H}_{M}$ is finite and such a partition always exist. This is the case considered in [Me]. The following proposition is an obvious generalization of this result of Melrose.

Proposition 7.1. Suppose that $M$ is a manifold with corners, and the set $\mathcal{H}_{M}$ of boundary hypersurfaces admits a finite partition as above. Then it is possible to construct a closed manifold $\widetilde{M}$ by gluing $2^{N}$ copies of $M$ along boundary hypersurfaces.

Proof. It is by induction on $N$. Let $M^{\prime}$ be a copy of $M$ with the same partition of the set $\mathcal{H}=\mathcal{H}_{M^{\prime}}=\mathcal{H}_{M}$ of boundary hypersurfaces. Glue $M$ and $M^{\prime}$ along the elements of $\mathcal{H}_{1} \subset \mathcal{H}$. We claim that $M \cup M^{\prime}$ is a manifold with corners. In fact, the interior points of the $H \in \mathcal{H}_{1}$ are manifold points of $M \cup M^{\prime}$, i.e., have euclidean neighborhoods. We need to check that boundary points of these hypersurfaces in $\mathcal{H}_{1}$, i.e., corner points of $M$, are also corner points of $M \cup M^{\prime}$. Let $H \in \mathcal{H}_{1}$ and $p$ in the boundary of $H$. Suppose that $p$ is of $\operatorname{rank} i$ in $M$. Then $i \geq 2$. Since all 
the boundary hypersurfaces of $M$ are embedded, there exist $i$ different hypersurfaces $H_{1}=H, \ldots, H_{i}$ such that $p \in H_{1} \cap \cdots \cap H_{i}$. By the assumption on the partition, the hypersurfaces in $\mathcal{H}_{1}$ are disjoint. This implies that $H_{2}, \ldots, H_{i}$ do not belong to $\mathcal{H}_{1}$. Then it is clear that after the gluing along $H_{1}, p$ has a chart in $M \cup M^{\prime}$ of the form $\mathbb{R}^{m-i+1} \times \mathbb{R}_{\geq 0}^{i-1}$ and becomes a point of rank $i-1$.

We claim that $\mathcal{H}_{M \cup M^{\prime}}$ admits a partition in $N-1$ subsets, each consisting of disjoint boundary hypersurfaces.

For every $j>1$, divide $\mathcal{H}_{j}$ into two subsets:

$$
\mathcal{H}_{j}=\mathcal{H}_{j, 1} \coprod \mathcal{H}_{j, 2},
$$

where $\mathcal{H}_{j, 1}=\left\{H \in \mathcal{H}_{j} \mid H \cap Z=\emptyset\right.$ for all $\left.Z \in \mathcal{H}_{1}\right\}$, and the $\mathcal{H}_{j, 2}$ is the complement. The elements of $\mathcal{H}_{j, 1}$ and their homologues in $M^{\prime}$ form a set of disjoint boundary hypersurfaces of $M \cup M^{\prime}$, say $\mathcal{H}_{j, 1}^{\prime \prime}$.

On the other hand, if $H \in \mathcal{H}_{j, 2}$, there exists $Z \in \mathcal{H}_{1}$ such that $H \cap Z \neq \phi$. For any such $Z, H \cap Z$ is a boundary hypersurface of $H$ and $Z$, and is equal to $H \cap H^{\prime}$, where $H^{\prime}$ is the homologue of $H$ on $M^{\prime}$. As observed earlier, the assumption on embeddedness of the boundary hypersurfaces of $M$ implies that the boundary hypersurfaces of $H$ are also embedded. Then the gluing of $M \cup M^{\prime}$ induces one of $H$ and $H^{\prime}$ along their intersection, which is similarly a manifold with corners, locally euclidean around an interior point of $H \cap H^{\prime}$. In particular, $H \cup H^{\prime}$ is a boundary hypersurface of $M \cup M^{\prime}$. Let $\mathcal{H}_{j, 2}^{\prime \prime}$ be the set of these glued up boundaries of $M \cup M^{\prime}$. They are disjoint since two elements of $\mathcal{H}_{j}$ are disjoint. Let $\mathcal{H}_{j}^{\prime \prime}=\mathcal{H}_{j, 1}^{\prime \prime} \cup \mathcal{H}_{j, 2}^{\prime \prime}$. Clearly hypersurfaces in $\mathcal{H}_{j}^{\prime \prime}$ are disjoint.

Since every boundary hypersurface of $M \cup M^{\prime}$ belongs to a unique $\mathcal{H}_{j}^{\prime \prime}$ for $j \geq 2$, we have a partition of $\mathcal{H}_{M \cup M^{\prime}}$ in $N-1$ subsets:

$$
\mathcal{H}_{M \cup M^{\prime}}=\coprod_{2 \leq j \leq N} \mathcal{H}_{j}^{\prime \prime}
$$

If $N=1$, then $M$ is a manifold with boundary and the previous construction provides the desired manifold $\widetilde{M}=M \cup M^{\prime}$. We can now use an induction hypothesis, which implies that we can glue $2^{N-1}$ copies of $M \cup M^{\prime}$ to obtain a closed manifold $\widetilde{M}$. Altogether, $\widetilde{M}$ is constructed by gluing $2^{N}$ copies of $M$.

q.e.d.

Remark 7.2. In a corner of rank $r$, there are $r$ boundary hypersurfaces with a non-empty intersection, hence $N \geq r k(M)$. The number $N$ depends on the partition. When $M$ is compact, the maximum value of $N$ is the number $N^{\prime}$ of boundary hypersurfaces, hence $\operatorname{rk}(M) \leq N \leq N^{\prime}$.

Proposition 7.3. If $M$ is $C^{\infty}$ (resp. real analytic), then so is $\widetilde{M}$. Moreover, if a group $H$ acts on $M$, then this action extends to one on 
$\tilde{M}$. The extended action is smooth (resp. real analytic) if $H$ is a Lie group and the given action on $M$ is so.

Proof. This follows from the construction: around a smooth point $x$ of $H \cap H^{\prime}$, the local charts in $M$ and $M^{\prime}$ are obtained from one another by a "reflection principle" with respect to $H \cap H^{\prime}$. These charts glue into a neighborhood of $x$ in $\widetilde{M}$, which is $C^{\infty}$ (resp. real analytic) if $M$ is so. To see that a group action on $M$ extends to $\widetilde{M}$, we note that for any two copies of $M$ in $\widetilde{M}$, the group actions on them agree on their intersection, and the combined action on $\widetilde{M}$ gives the extension. q.e.d.

Proposition 7.4. The closed manifold $\widetilde{M}$ constructed in Proposition 7.1 admits a $(\mathbb{Z} / 2 \mathbb{Z})^{N}$-action such that the quotient of $\widetilde{M}$ by $(\mathbb{Z} / 2 \mathbb{Z})^{N}$ is equal to $M$. If $M$ admits a group action by $H$ as in Proposition 7.3, then the extended $H$-action commutes with this $(\mathbb{Z} / 2 \mathbb{Z})^{N}$-action.

Proof. We prove this by induction. When $N=1, M$ is a manifold with boundary, and $\widetilde{M}$ is obtained from $M$ by doubling across the boundary and clearly admits a $\mathbb{Z} / 2 \mathbb{Z}$-action.

As in the proof of Proposition 7.1, $M \cup M^{\prime}$ admits a partition of $N-1$ subsets, and $\widetilde{M}$ is glued from $2^{N-1}$ copies of $M \cup M^{\prime}$. By induction, $\widetilde{M}$ admits a $(\mathbb{Z} / 2 \mathbb{Z})^{N-1}$-action, and the quotient by this group is equal to $M \cup M^{\prime}$. By Proposition 7.3 , the $\mathbb{Z} / 2 \mathbb{Z}$-action on $M \cup M^{\prime}$ extends to an action on $\widetilde{M}$. This $\mathbb{Z} / 2 \mathbb{Z}$-action commutes with the $(\mathbb{Z} / 2 \mathbb{Z})^{N-1}$ action on $\widetilde{M}$ by induction. Hence $\widetilde{M}$ admits a $(\mathbb{Z} / 2 \mathbb{Z})^{N}$-action, and the quotient of $\widetilde{M}$ by $(\mathbb{Z} / 2 \mathbb{Z})^{N}$ is equal to the quotient $M \cup M^{\prime}$ by $\mathbb{Z} / 2 \mathbb{Z}$ and hence equal to $M$.

To show that the extended $H$-action on $\tilde{M}$ commutes with $(\mathbb{Z} / 2 \mathbb{Z})^{N}$, we note that $(\mathbb{Z} / 2 \mathbb{Z})^{N}$ interchanges different copies of $M$. Since the $H$-action on the all the copies of $M$ is the same, the extended $H$-action commutes with the $(\mathbb{Z} / 2 \mathbb{Z})^{N}$-action.

q.e.d.

\section{Construction of $\Gamma \backslash \bar{G}^{B S O}$ and $\Gamma \backslash \bar{X}^{B S O}$}

It is proved in $\S 6$ that $\bar{G}^{B S}, \Gamma \backslash \bar{G}^{B S}, \bar{X}^{B S}, \Gamma \backslash \bar{X}^{B S}$ are real analytic manifolds with corners. In this section, we study structure of their boundary faces and show that $2^{r}$ copies of each of them can be glued into a closed real analytic manifold by the method of $\S 7$, where $r$ is the $\mathbb{Q}$-rank of $\mathbf{G}$, which is defined earlier as the maximum of $\operatorname{dim} A_{P}$ for all parabolic $\mathbb{Q}$-subgroups $\mathbf{P}$ of G. By Remark 7.2, this is the least number of copies needed to glue into a closed manifold.

Lemma 8.1. For every parabolic $\mathbb{Q}$-subgroup $\mathbf{Q}$, the closure $\overline{e(Q)}$ of the boundary face $e(Q)$ in $\bar{G}^{B S}$ is a (closed) boundary face of codimension $\operatorname{dim} A_{Q}$ and is hence a real analytic submanifold with corners. 
Proof. From the definition of convergence of sequences of boundary points in $\bar{G}^{B S}$,

$$
\overline{e(Q)}=e(Q) \cup \coprod_{P \subset Q} e(P) .
$$

Therefore, $\overline{e(Q)}$ is covered by the corners $G(P)$ for all $P \subseteq Q$. In each corner $G(P)$,

$$
\overline{e(Q)} \cap G(P)=\coprod_{P \subseteq R \subseteq Q} e(R) \subset G \cup \coprod_{P \subseteq R} e(R) .
$$

By Lemmas 3.5 and 3.6, in the decomposition $G(P)=N_{P} \times \overline{A_{P, Q}} \times$ $\overline{A_{Q}} \times M_{P} K$,

$$
\overline{e(Q)} \cap G(P)=N_{P} \times \overline{A_{P, Q}} \times\left\{o_{Q}\right\} \times M_{P} K,
$$

which is clearly a real analytic submanifold with corners in $G(P)$ of codimension $\operatorname{dim} A_{Q}$. This implies that $\overline{e(Q)}$ is a real analytic submanifold with corners in $\bar{G}^{B S}$ of codimension $\operatorname{dim} A_{Q}$.

q.e.d.

Corollary 8.2. The rank of $\bar{G}^{B S}$ as a manifold with corners is equal to the $\mathbb{Q}$-rank of the algebraic group $\mathbf{G}$.

Lemma 8.3. The boundary hypersurfaces of $\bar{G}^{B S}$ are the $\overline{e(Q)}$, where $\mathbf{Q}$ are parabolic $\mathbb{Q}$-subgroups of rank $1, \operatorname{dim} A_{Q}=1$, i.e., $\mathbf{Q}$ are proper maximal parabolic $\mathbb{Q}$-subgroups, and they are embedded as defined in $\S 7$.

Proof. The first statement clearly follows from Lemma 8.1. To prove the second statement, we note that for every parabolic $\mathbb{Q}$-subgroup $\mathbf{P}$ of rank $i$, i.e, $\operatorname{dim} A_{P}=i$, there are exactly $i$ maximal proper parabolic $\mathbb{Q}$-subgroups containing P. In fact, this fact follows from the one-toone correspondence in $\S 2$ between subsets of the set of simple roots $\Delta\left(P, A_{P}\right)$ and parabolic $\mathbb{Q}$-subgroups containing P. This implies that every point in $e(P)$, which has rank $i$ by Lemma 8.1, is contained in exactly $i$ different boundary hypersurfaces. This proves the all boundary hypersurfaces are embedded. q.e.d.

Lemma 8.4. Let $\mathbf{Q}_{1}, \mathbf{Q}_{2}$ be two parabolic $\mathbb{Q}$-subgroups and $\mathbf{P}=\mathbf{Q}_{1} \cap$ $\mathbf{Q}_{2}$. If $\mathbf{P}$ is not a parabolic $\mathbb{Q}$-subgroup, the boundary faces $\overline{e\left(Q_{1}\right)}, \overline{e\left(Q_{2}\right)}$ are disjoint. Otherwise, $\overline{e\left(Q_{1}\right)} \cap \overline{e\left(Q_{2}\right)}=\overline{e(P)}$.

Proof. This follows from the equation $\overline{e\left(Q_{i}\right)}=\coprod_{\mathbf{P} \subseteq \mathbf{Q}_{i}} e(P)$. q.e.d.

Lemma 8.5. Let $r$ be the $\mathbb{Q}$-rank of $\mathbf{G}$. Then there exists a partition of the set $\mathcal{H}_{\bar{G}^{B S}}$ of boundary hypersurfaces of $\bar{G}^{B S}$ into $r$ parts, $\mathcal{H}_{\bar{G}^{B S}}=$ $\coprod_{j=1}^{r} \mathcal{H}_{j}$, such that for every $j$, the hypersurfaces in $\mathcal{H}_{j}$ are disjoint.

Proof. Let $\mathbf{P}$ be any minimal parabolic $\mathbb{Q}$-subgroup of $\mathbf{G}$. Then $\operatorname{dim} A_{P}=r$. By the one-to-one correspondence between subsets of 
the simple roots $\Delta\left(P, A_{P}\right)$ and parabolic subgroups containing $\mathbf{P}$, there are exactly $r$ maximal parabolic $\mathbb{Q}$-subgroups containing $\mathbf{P}$.

Fix a minimal parabolic subgroup $\mathbf{P}$ and denote the maximal parabolic $\mathbb{Q}$-subgroups containing $\mathbf{P}$ by $\mathbf{Q}_{1}, \ldots, \mathbf{Q}_{r}$.

For any other minimal parabolic $\mathbb{Q}$-subgroup $\mathbf{P}^{\prime}$, it is known that there exists an element $g \in \mathbf{G}(\mathbb{Q})$ such that $\mathbf{P}^{\prime}=g \mathbf{P} g^{-1}$. Under this conjugation, the maximal parabolic subgroups containing $\mathbf{P}$ are mapped to the maximal parabolic subgroups containing $\mathbf{P}^{\prime}$. Denote them by $\mathbf{Q}_{1}^{\prime}=g \mathbf{Q}_{1} g^{-1}, \ldots, \mathbf{Q}_{r}^{\prime}=g \mathbf{Q}_{r} g^{-1}$. We claim that this numbering of the maximal parabolic subgroups containing $\mathbf{P}^{\prime}$ is independent of the choice of the element $g \in \mathbf{G}(\mathbb{Q})$. In fact, $g$ is unique up to an element of $\mathbf{P}(\mathbb{Q})$. Since the conjugation by an element of $\mathbf{P}(\mathbb{Q})$ leaves all $\mathbf{Q}_{1}, \ldots, \mathbf{Q}_{r}$ stable, the claim is proved.

Now define $\mathcal{H}_{j}$ to be the set of the boundary hypersurfaces $\overline{e\left(Q_{j}^{\prime}\right)}$ for all minimal rational parabolic subgroups $\mathbf{P}^{\prime}$. Since every maximal parabolic $\mathbb{Q}$-subgroup contains a minimal rational parabolic subgroup, $\mathcal{H}_{1}, \ldots, \mathcal{H}_{r}$ forms a partition of the set of boundary hypersurfaces of $\bar{G}^{B S}$.

By Lemma 8.4, the hypersurfaces in each $\mathcal{H}_{j}$ are disjoint, since no two $\mathbf{Q}_{j}^{\prime}$ contain a parabolic $\mathbb{Q}$-subgroup. $\quad$ q.e.d.

The manifold $\bar{G}^{B S}$ has infinitely many boundary faces, since there are infinitely many parabolic $\mathbb{Q}$-subgroups of $\mathbf{G}$. On the other hand, the following is true.

Theorem 8.6. Let $r$ be the $\mathbb{Q}$-rank of $\mathbf{G}$. Then $2^{r}$ copies of the BorelSerre partial compactification $\bar{G}^{B S}$ can be glued into a closed analytic manifold by the methods in $\S 7$. This closed analytic manifold is denoted by $\bar{G}^{B S O}$ and admits a $(\mathbb{Z} / 2 \mathbb{Z})^{r}$-action whose quotient is equal to $\bar{G}^{B S}$.

Proof. By Lemma 8.5, the set of boundary hypersurfaces $\mathcal{H}_{\bar{G}^{B S}}$ admits a partition $\mathcal{H}_{1}, \ldots, \mathcal{H}_{r}$ such that the hypersurfaces in each $\mathcal{H}_{j}$ are disjoint. Proposition 7.1 or Proposition 7.6 shows that $2^{r}$ copies of $\bar{G}^{B S}$ can be glued into a closed analytic manifold. Since $\bar{G}^{B S}$ is a real analytic manifold with corners by Proposition 6.3, it follows from Proposition 7.3 that $\bar{G}^{B S O}$ is an analytic manifold. Proposition 7.4 gives the action of $(\mathbb{Z} / 2 \mathbb{Z})^{r}$.

q.e.d.

Remark 8.7. Let $X=G / K$. Denote the rank of $X$ by $r$. In [Os1], Oshima constructed a closed analytic manifold $\bar{X}^{O}$ which contains the union of $2^{r}$ copies of $X=G / K$ as an open dense subset such that the closure of each one is the maximal Satake compactification $\bar{X}_{\text {max }}^{S}$. It can be shown that $\bar{X}_{\max }^{S}$ is a real analytic manifold with corners of rank $r$ and $2^{r}$ copies of $\bar{X}_{\max }^{S}$ can be glued into a closed analytic manifold which 
is exactly the Oshima compactification $\bar{X}^{O}$. Due to this connection, the closed analytic manifold constructed in the above theorem is called the Borel-Serre-Oshima compactification of $G$ and hence denoted by $\bar{G}^{B S O}$.

Corollary 8.8. When $\Gamma$ is a neat arithmetic subgroup, $2^{r}$ copies of $\Gamma \backslash \bar{G}^{B S}$ can be glued into a closed analytic manifold, denoted by $\Gamma \backslash \bar{G}^{B S O}$, which admits a $(\mathbb{Z} / 2 \mathbb{Z})^{r}$-action whose quotient is equal to $\Gamma \backslash \bar{G}^{B S}$.

Proof. By the proof of Proposition 6.4, $\Gamma$ acts on $\bar{G}^{B S}$ by real analytic diffeomorphism. By Proposition 7.3, this $\Gamma$-action extends to $\bar{G}^{B S O}$. Then the quotient of $\bar{G}^{B S O}$ by $\Gamma$ is a compact closed analytic manifold consisting of $2^{r}$ copies of $\Gamma \backslash \bar{G}^{B S}$. By Proposition 7.4 , the $(\mathbb{Z} / 2 \mathbb{Z})^{r}$ action on $\bar{G}^{B S O}$ commutes with $\Gamma$ and hence descends to the quotient by $\Gamma$.

q.e.d.

Remark 8.9. The above corollary can also be proved directly without using $\bar{G}^{B S O}$. In fact, under the $\Gamma$-action, the partition $\mathcal{H}_{1}, \ldots, \mathcal{H}_{r}$ of $\mathcal{H}_{\bar{G}^{B S}}$ is preserved and hence induces a partition into $r$ parts of $\mathcal{H}_{\Gamma \backslash \bar{G}^{B S}}$. Since $\Gamma \backslash \bar{G}^{B S}$ is compact and hence has only finitely many boundary faces, Proposition 7.1 (or 7.6) and Proposition 6.4 show that $2^{r}$ copies of $\Gamma \backslash \bar{G}^{B S}$ can be glued into a compact closed analytic manifold.

By the same method as above, we can prove the following result.

Theorem 8.10. Let $r$ be the $\mathbb{Q}$-rank of $\mathbf{G}$. Then $2^{r}$ copies of $\bar{X}^{B S}$ can be glued into a closed analytic manifold $\bar{X}^{B S O}$, and $2^{r}$ copies of $\Gamma \backslash \bar{X}^{B S}$ can be glued into a closed compact analytic manifold $\Gamma \backslash \bar{X}^{B S O}$. Both $\bar{X}^{B S O}$ and $\Gamma \backslash \bar{X}^{B S O}$ admit a $(\mathbb{Z} / 2 \mathbb{Z})^{r}$-action whose quotients are $\bar{X}^{B S}$ and $\Gamma \backslash \bar{X}^{B S}$ respectively.

\section{References}

[A] N.D. Allan, The problem of the maximality of arithmetic groups, in 'Algebraic groups and discontinuous subgroups', Proc. Symp. Pure Math., IX (A. Borel and G.D. Mostow, eds.), AMS, 1966, MR 0205997, Zbl 0192.12901.

[BB] W.L. Baily \& A. Borel, Compactification of arithmetic quotients of bounded symmetric domains, Ann. of Math. 84 (1966) 442-528, MR 0216035, Zbl 0154.08602.

[Bo1] A. Borel, Introduction aux groupes arithmétiques, Hermann, Paris, 1969, MR 0244260, Zbl 0186.33202.

[Bo2] _ Density properties for certain subgroups of semisimple groups without compact factors, Ann. of Math. 72 (1960) 179-188, MR 0123639, Zbl 0094.24901. 
[Bo3] , Density and maximality of arithmetic subgroups, J. Reine Angew. Math. 224 (1966) 78-89, MR 0205999, Zbl 0158.03105.

[Bo4] Sous-groupes discrets de groupes semi-simples, Séminaire Bourbaki, Exp. 358 (1968/69), Lect. Notes Math. 179 (1971) 199-216, MR 0272579, Zbl 0225.22017.

[Bo5] Stable real cohomology of arithmetic groups, Ann. Sci. Ec. Norm. Super. 7 (1974) 235-272, MR 0387496, Zbl 0316.57026.

[Bo6] , Notes of a seminar on compactifications of symmetric spaces at IAS, Princeton, 1987.

[Bo7] - Some metric properties of arithmetic quotients of symmetric spaces and an extension theorem, J. Differential Geom. 6 (1972) 543-560, MR 0338456, Zbl 0249.32018.

[BJ1] A. Borel \& L. Ji, Compactifications of symmetric and locally symmetric spaces, Math. Research Letters 9 (2002) 725-739, MR 1906074, Zbl 1027.22011.

[BJ2] Compactifications of symmetric spaces, preprint.

[BS] A. Borel \& J.-P. Serre, Corners and arithmetic groups, Comment. Math. Helv. 48 (1973) 436-491, MR 0387495, Zbl 0274.22011.

[BT] A. Borel \& J. Tits, Groupes réductifs, Publ. Sci. IHES 27 (1965) 55-150, MR 0207712, Zbl 0145.17402.

[Bu] N. Bourbaki, Éléments de Mathématique, Livre VI, Intégration, Hermann, Paris, 1963, MR 0179291, Zbl 0156.03204.

[DP] C. De Concini \& C. Procesi, Complete symmetric varieties, in 'Invariant Theory', Springer Lecture Notes, 996 (1983) 1-44, MR 0718125, Zbl 0581.14041.

[Fu] H. Furstenberg, A Poisson formula for semi-simple Lie groups, Ann. of Math. 72 (1963) 335-386, MR 0146298, Zbl 0192.12704.

[GHM] M. Goresky, G. Harder, \& R. MacPherson, Weighted cohomology, Invent. Math. 116 (1994) 139-213, MR 1253192, Zbl 0849.11047.

[Gr] P. Griffiths, Periods of integrals on algebraic manifolds: Summary of main results and discussion of open problems, Bull. Amer. Math. Soc. 76 (1970) 228-296, MR 0258824, Zbl 0214.19802.

[GJT] Y. Guivarc'h, L. Ji, \& J.C. Taylor, Compactifications of symmetric spaces, in 'Progress in Math.', 156, Birkhauser, Boston, 1998, MR 1633171, Zbl 1053.31006.

[HC] Harish-Chandra, Automorphic forms on semisimple Lie groups, Lect. Notes in Math., 62, Springer-Verlag, 1968, MR 0232893, Zbl 0186.04702.

[Ji] L. Ji, The trace class conjecture for arithmetic groups, J. Differential Geom. 48 (1998) 165-203, MR 1622604, Zbl 0926.11034.

[JM] L. Ji \& R. MacPherson, Geometry of compactifications of locally symmetric spaces, Ann. Inst. Fourier (Grenoble) 52(2) (2002) 457-559, MR 1906482, Zbl 1017.53039.

[KK] M. Kashiwara, A. Kowata, K. Minemura, K. Okamoto, T. Oshima, \& M. Tanaka, Eigenfunctions of invariant differential operators on a symmetric space, Ann. of Math. 107 (1978) 1-39, MR 0485861, Zbl 0377.43012.

[KU] K. Kato \& S. Usui, Borel-Serre spaces and spaces of SL(2)-orbits, in 'Algebraic geometry 2000', Azumino (Hotaka), 321-382, Adv. Stud. Pure Math., 36, Math. Soc. Japan, Tokyo, 2002, MR 1971520, Zbl 1071.14014. 
[KM] D. Kazhdan \& G.A. Margulis, A proof of Selberg's hypothesis (Russian), Math. Sbornik N.S. 75 (1968) 163-168, MR 0223487, Zbl 0241.22024.

[Ma] R. MacPherson, Seminar notes on locally symmetric spaces and their compactifications, taken by M. Goresky, 1985, 1989.

[Me] R. Melrose, A private letter, 1998.

[Os1] T. Oshima, A realization of Riemannian symmetric spaces, J. Math. Soc. Japan 30 (1978) 117-132, MR 0477175, Zbl 0364.43010.

[Os2] _ A realization of semisimple symmetric spaces and construction of boundary value maps, Adv. Studies in Pure Math., 14, Representations of Lie groups, 1988, 603-650, MR 1039854, Zbl 0729.22012.

[Os3] - Harmonic analysis on semisimple symmetric spaces, Sugaku Expositions 12 (2002) 151-170, MR 1944133, Zbl 0606.43006.

[OSS] T. Oshima \& J. Sekiguchi, Eigenspaces of invariant differential operators on an affine symmetric spaces, Invent. Math. 57 (1980) 1-81, MR 0564184, Zbl 0434.58020.

[OW] M.S. Osborne \& G. Warner, The theory of Eisenstein systems, Academic Press, 1981, New York, MR 0643242, Zbl 0489.43009.

[Sap] L. Saper, Tilings and finite energy retractions of locally symmetric spaces, Comment. Math. Helv. 72 (1997) 167-202, MR 1470087, Zbl 0890.22003.

[Sa1] I. Satake, On representations and compactifications of symmetric spaces, Ann. of Math. 71 (1960) 77-110, MR 0118775, Zbl 0094.34603.

[Sa2] On compactifications of the quotient spaces for arithmetically defined discontinuous groups, Ann. of Math. 72 (1960) 555-580, MR 0170356, Zbl 0146.04701.

[We] U. Weselmann, Twisted topological trace formula, Notes of a talk taken by M. Goresky, Oberwolfach, Germany, 1997.

[Zu1] S. Zucker, L $L_{2}$ cohomology of warped products and arithmetic groups, Invent. Math. 70 (1982) 169-218, MR 0684171, Zbl 0508.20020.

[Zu2] , Satake compactifications, Comment. Math. Helv. 58 (1983) 312-343, MR 0705539, Zbl 0565.22009.

[Zu3] - On the reductive Borel-Serre compactification: $L^{p}$-cohomology of arithmetic groups, Amer. J. Math. 123 (2001) 951-984, MR 1854116, Zbl 0998.14013.

School of Mathematics Institute For AdVANCED Studies Princeton, NJ 08540

Department of Mathematics UNIVERSITY OF MiCHIGAN ANN ARBOR, MI 48109

E-mail address: lji@umich.edu 